LAWRENCE LIVERMORE N A TIO N A L LABORATORY

\title{
Computed Tomography Analysis of NASA BSTRA Balls
}

R. L. Perry, D. J. Schneberk, R. R. Thompson

November 9, 2004 
This document was prepared as an account of work sponsored by an agency of the United States Government. Neither the United States Government nor the University of California nor any of their employees, makes any warranty, express or implied, or assumes any legal liability or responsibility for the accuracy, completeness, or usefulness of any information, apparatus, product, or process disclosed, or represents that its use would not infringe privately owned rights. Reference herein to any specific commercial product, process, or service by trade name, trademark, manufacturer, or otherwise, does not necessarily constitute or imply its endorsement, recommendation, or favoring by the United States Government or the University of California. The views and opinions of authors expressed herein do not necessarily state or reflect those of the United States Government or the University of California, and shall not be used for advertising or product endorsement purposes.

This work was performed under the auspices of the U.S. Department of Energy by University of California, Lawrence Livermore National Laboratory under Contract W-7405-Eng-48. 


\title{
Computed Tomography Analysis of NASA BSTRA Balls
}

\author{
Serial Numbers 6-92-003 to 6-92-017 \\ Roger L. Perry, Lawrence Livermore National Laboratory
}

\section{Executive Summary}

Fifteen 1.25 inch BSTRA balls were scanned with the high energy computed tomography system at LLNL. This system has a resolution limit of approximately 210 microns. A threshold of 238 microns (two voxels) was used, and no anomalies at or greater than this were observed.

\section{Introduction \& Scan Parameters}

At the request of NASA personnel, we performed digital radiography/computed tomography scanning on fifteen BSTRA Balls. All BSTRA Ball specimens were scanned on the same HECAT (high energy computed tomography) system as used for all previous BSTRA scans (some 45 units total). This report addresses the scan of serial numbers 6-92-003 to 6-92-017. This particular system employs a 9 meV LINAC as the $\mathrm{x}$ ray source and a THALES 12 x 16 inch 14-bit Amorphous Silicon panel as the detector.

Figure 1 contains two images of the system as fielded in the $9 \mathrm{MeV}$ bay. The LINAC is in the right portion of the picture. The black panels in the blue frame constitute the High Energy collimator developed specifically for High Energy digital radiography/computed tomography scanning (referred to here as Stonehenge II). The holes in the collimator panels are beveled to match the distribution of the x-rays from the LINAC, and are sized to just subtend the active area of the THALES Amorphous Silicon panel (see Figure 2). Consequently the source to detector distance is restricted to a few positions. Nominally our source to detector distance is 6 meters, with the object approximately one-half meter away from the detector (see below).

The specimen holder and manipulator consist of a translate-rotate assembly on a NEWPORT air bearing table. The stages are NEWPORT RV160PP for rotation and NEWPORT IMS400CC for translation (see Figure 2). Both are interfaced through an ESP7000 controller connected to our data acquisition computer over USB. The detector holder also resides on this table and includes pitch, roll and yaw adjustments for aligning the panel to the plane of the rotational table and normal to the $\mathrm{x}$-ray beam.

\section{Scan procedure}

All the scans were performed with the Stonehenge II collimator. The source to detector distance is $5743 \mathrm{~mm}$. The object is $360 \mathrm{~mm}$. from the detector, putting the source to object distance at $5383 \mathrm{~mm}$. With this geometry, the maximum cone angle is approximately 2 degrees. For the objects we are scanning the cone angle ranges from 0.5 to 0.75 degrees. We are running the Am-Si panel in an un-binned format. Detector pixel size is $0.127 \mathrm{~mm}$, and with the magnification of this configuration, puts the voxel size for the reconstructed cubes of data at $0.119 \mathrm{~mm}$. We have measured the spatial 
resolution of this data at approximately 210 um, using the ASTM PSF (American Society for Testing and Materials, Point Spread Function) estimation method on a tantalum cylinder containing holes. As corroboration of spatial resolution down to this scale, the cylinder contained some 250 um holes which could be seen in different slice planes of the three dimensional volume. The usual caveats for spatial resolution apply: it is possible to see high-contrast features which are smaller than $200 \mathrm{um}$. however, low contrast features will "just be imaged" at this spatial scale. The computed tomography scan of these particular items involved 360 views over 360 degrees. Digital radiographs (figure 3) were reconstructed into three dimensional volumes using LLNL computed tomography Preprocessing and reconstruction software. The data set associated with each of the balls including reconstructions, original radiographs and intermediate processing steps takes about 1.5 GB of disk space.

All handling of the BSTRA balls was done per the requirements jointly arrived at between LLNL and NASA. These include a two person rule when ever the locked container where the balls were stored was opened and the scanning of only one ball at a time. A thin plastic cylinder (figure 4) was fabricated to facilitate the scan of these 1.25 inch balls. All balls were scanned using the same parameters: 360 angular views, unfiltered x-ray source due to the material properties and 6 frame averages to minimize image noise. Reconstructions were performed using the CBP (convolution back projection) algorithm. Each of the balls were scanned individually and the data stored in a directory named with the serial number of the ball.

\section{Analysis of three dimensional computed tomography Scan Data}

Each reconstructed data set was examined on a slice by slice basis for anomalies. For the purposes of quick review a standard data packet was prepared for each ball scanned: an attenuation radiograph (taken at the 0 deg position), shell extractions taken 5 and 20 pixels inside the outer edge, a $\mathrm{z}$ plane average of 5 slices taken at the center of the ball, and a y slice average of 5 slices taken at the center of the ball. There were no significant indications of defects or anomalies in any of the balls scanned. The criteria for a significant defect was the ability to see the defect in at least two adjacent voxels. The images from the "standard" data packet are included in this report for review.

\section{Summary}

We acquired three dimensional computed tomography volumetric inspection data for a series of 1.25 inch BSTRA Balls. From the three dimensional volume of data we examined numerous two dimensional slices from different orientations, and generated two "shell extractions". Each shell-extraction image is an interpolated shell of intensities but at different depths in the ball. A fit to the outside surface is used as the datum surface, which is then converted to boundary points and reduced in size for extractions at depths inside the Ball. These various slice and shell extraction methods are attempts (and have proven effective) at identifying and isolating various anomalies. The goal of this inspection is to locate and describe any anomalies within the balls.

While single voxel indications existed, we used the criteria that an indication of a defect had to be present in at least two adjacent voxels to be considered valid. There were no 
defects detected in the 15 balls scanned for this report at or greater than two voxels or 238 microns.

This work was performed under the auspices of the U.S. Department of Energy by University of California, Lawrence Livermore National Laboratory under Contract W7405-Eng-48. 

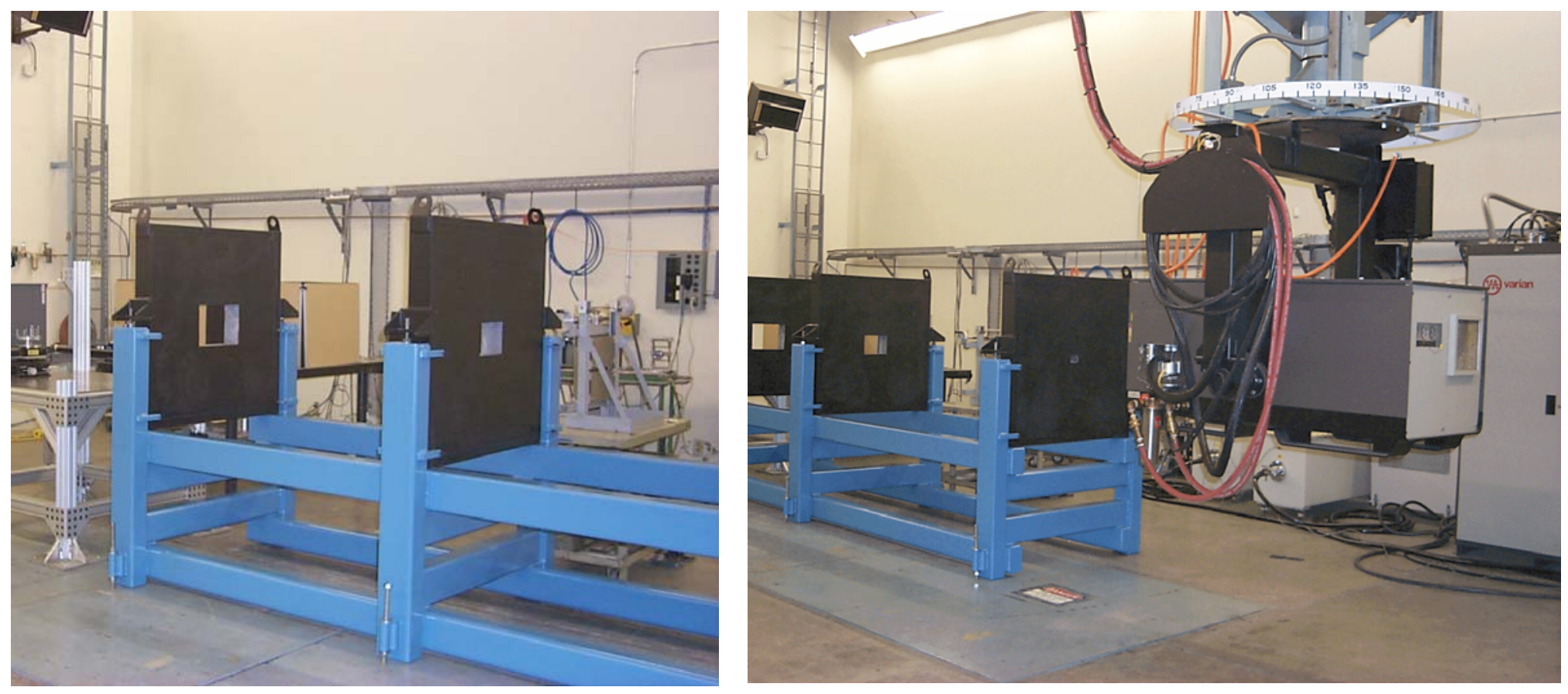

Figure 1 - Picture of HECAT components - LINAC \& Stonehenge II Collimator. 


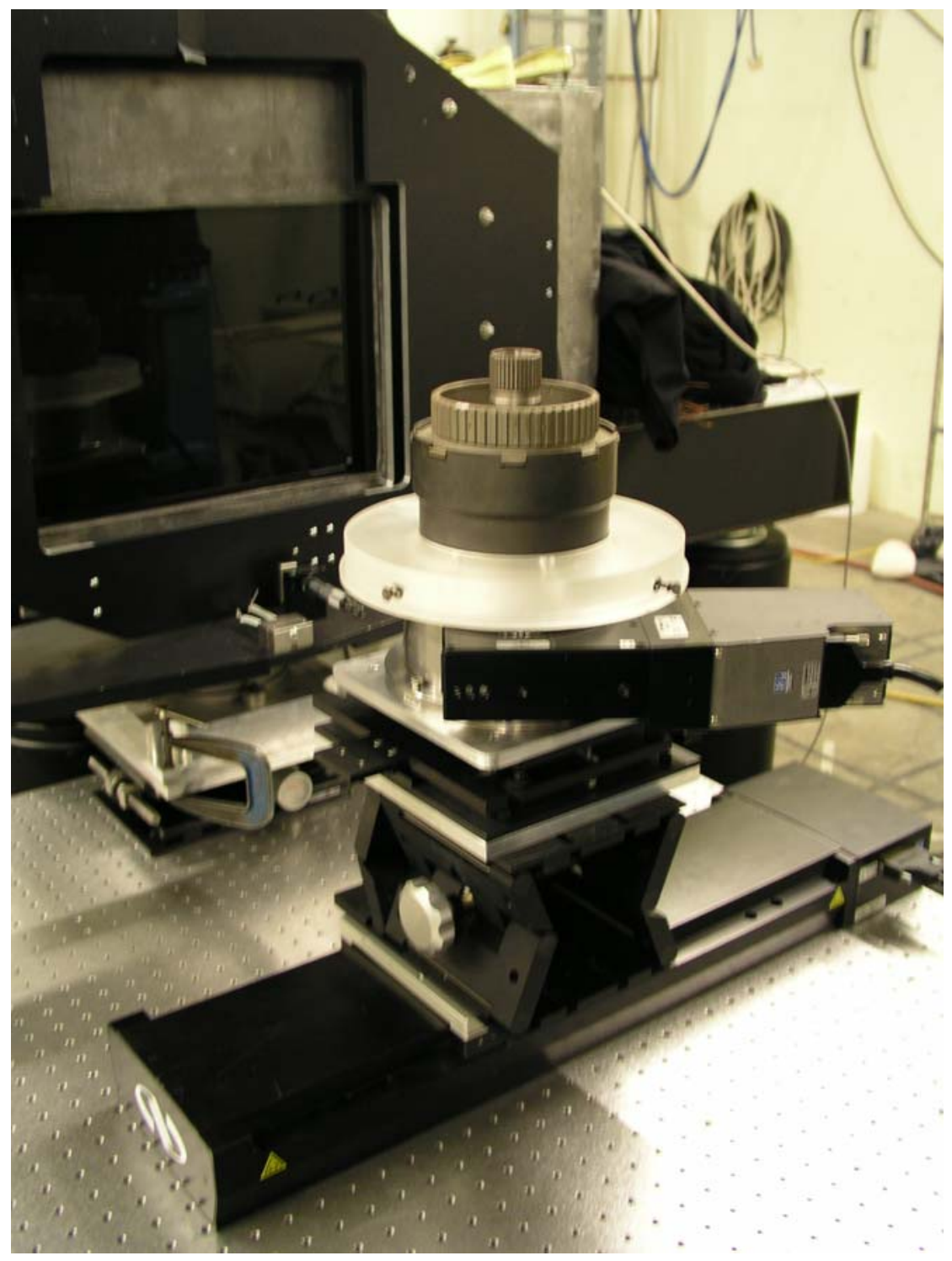

Figure 2 - Photo of Object positioning system and THALES 14-bit Am-Si panel with Industrial object loaded on rotary stage. 


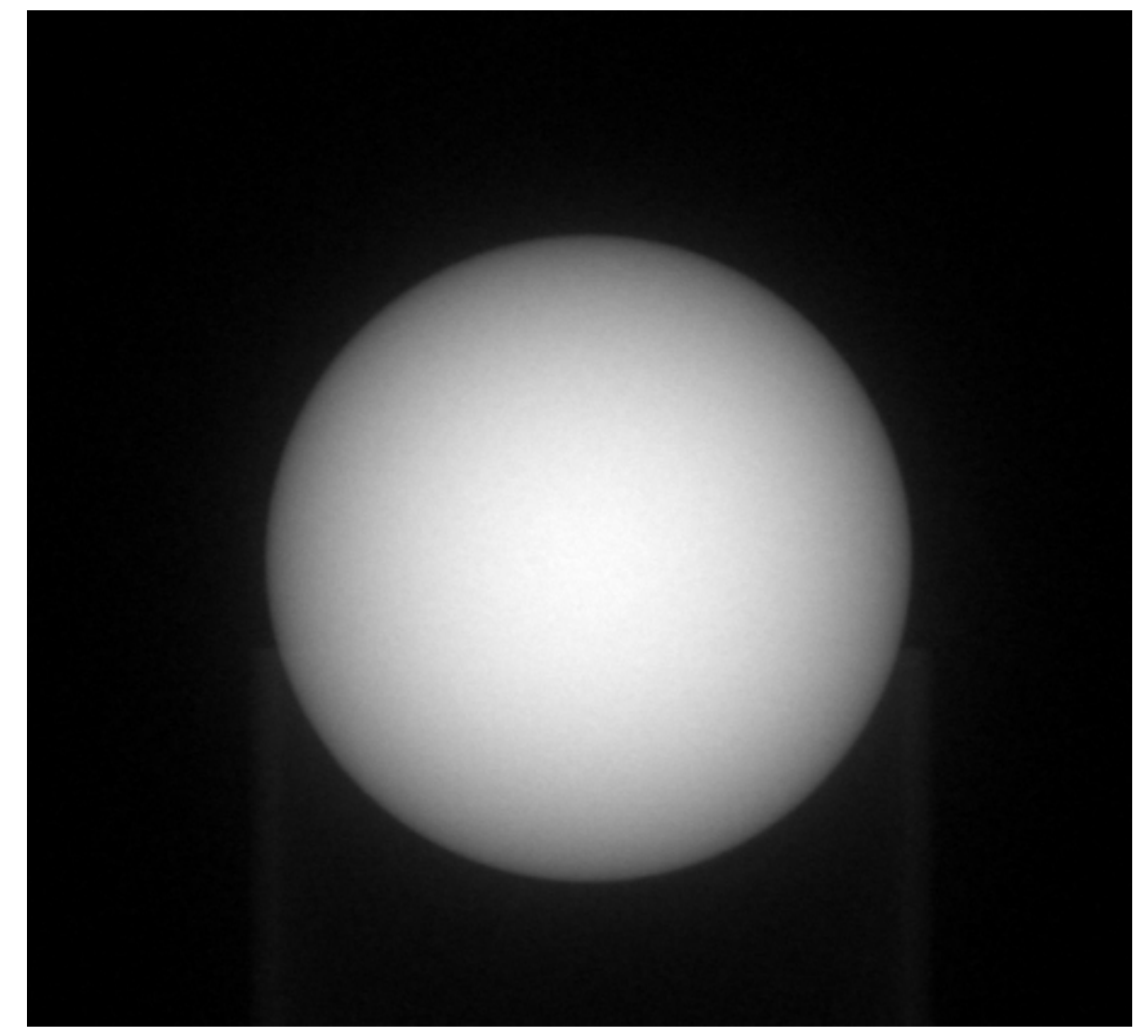

Figure 3 - Typical sample of an attenuation radiograph (1 of 360) derived from original scan data and used in reconstruction of $3 \mathrm{D}$ volume. 


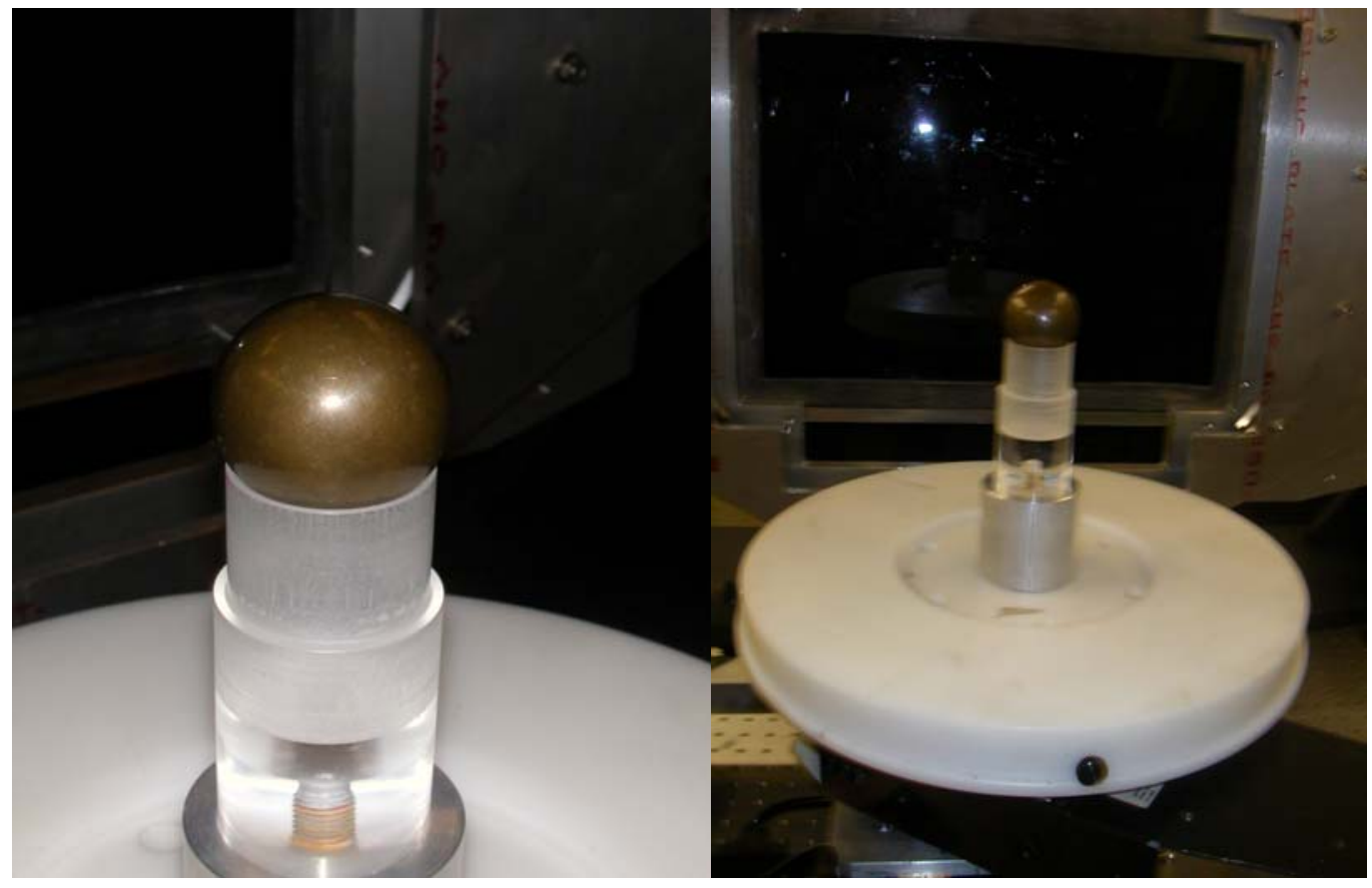

Figure 4 - Small geometry plastic holder designed to minimize impact on reconstruction of $3 \mathrm{D}$ data sets. 


\section{Representative Images:}

The following pages consist of a sampling of images taken from each of the BSTRA balls analyzed. They are organized with the identifying serial number at the top and sample images and descriptions of each. 


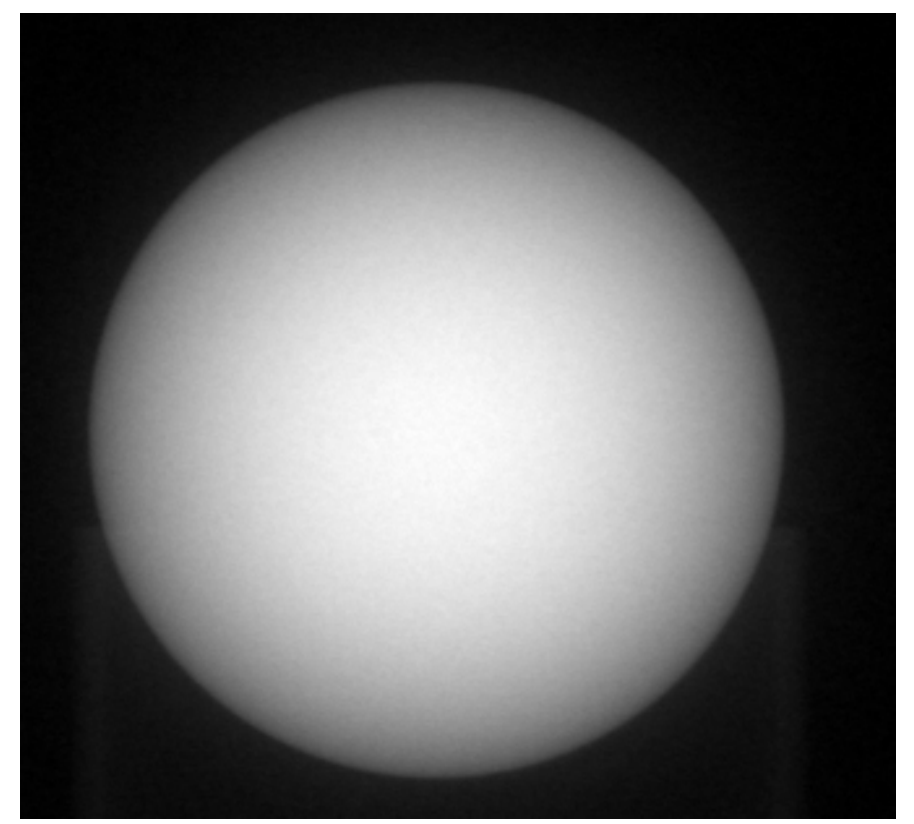

Attenuation radiograph, 0 degree.

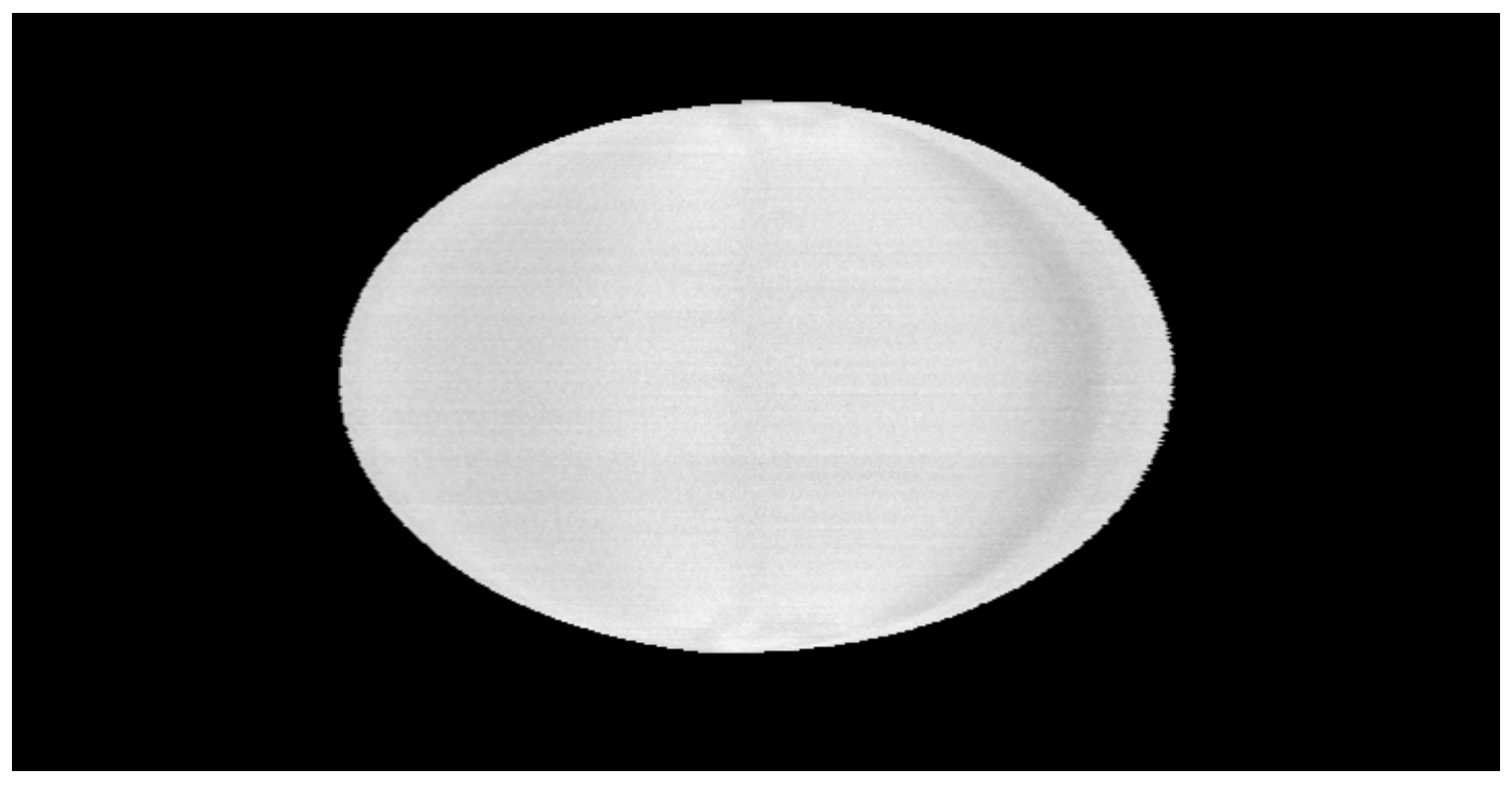

Shell extraction, 5 pixels inside of outer surface. 
SN 6-92-003

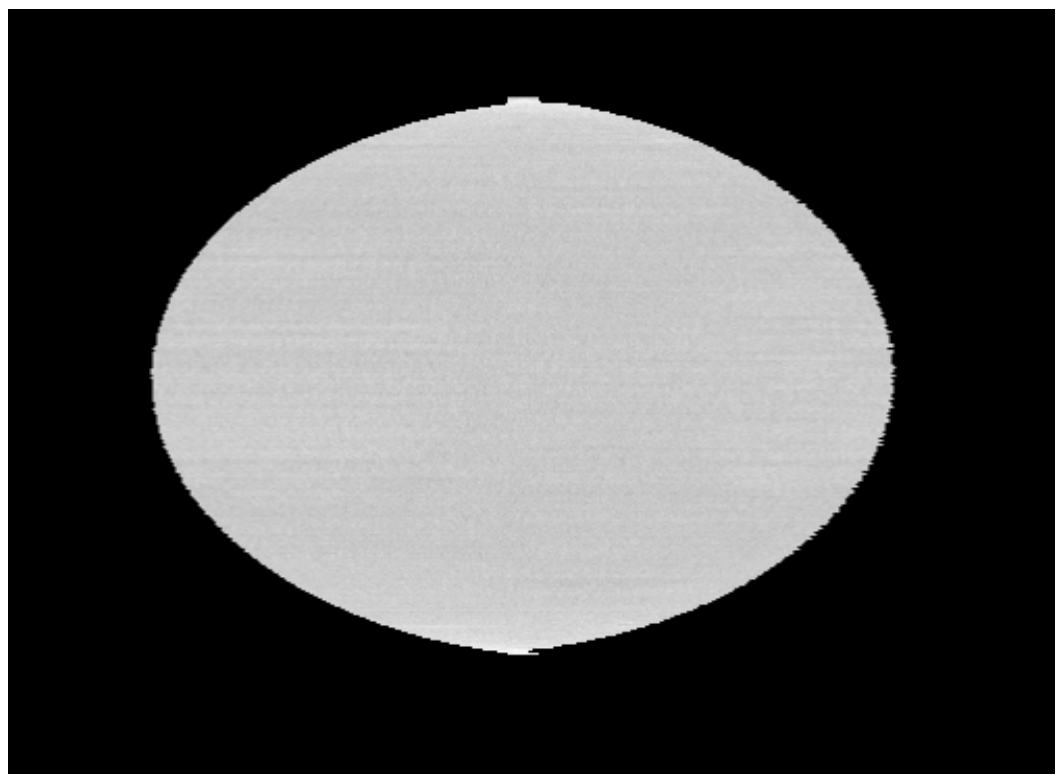

Shell extraction, 20 pixels inside of outer surface.

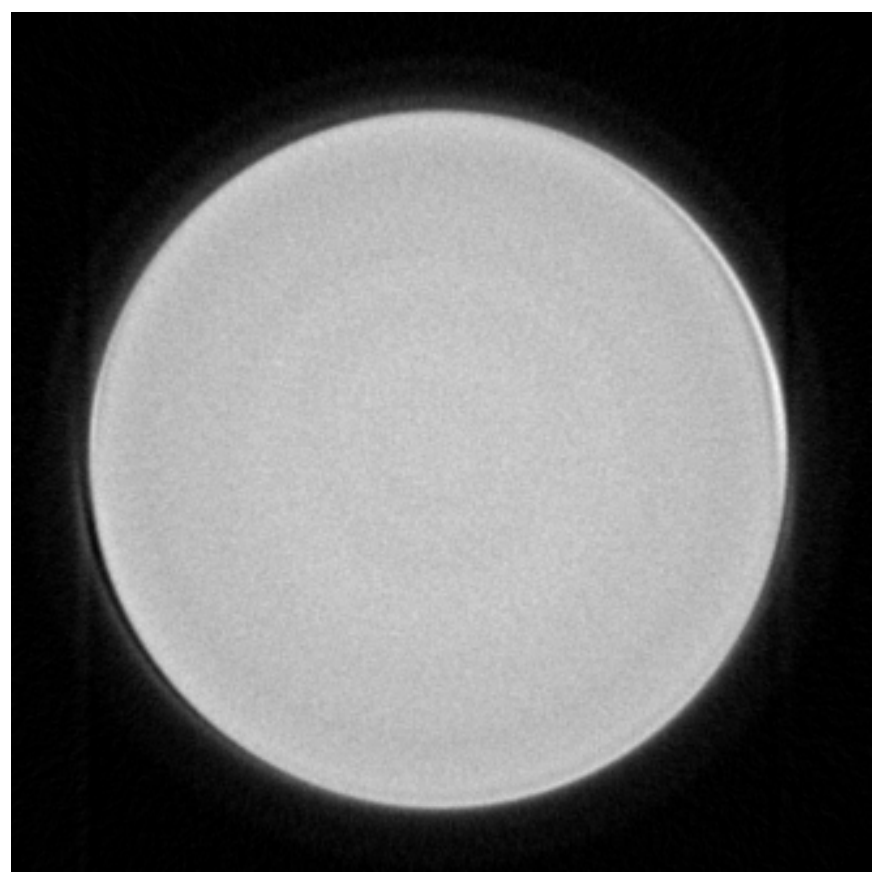

Z slice, average of slices 223-227.

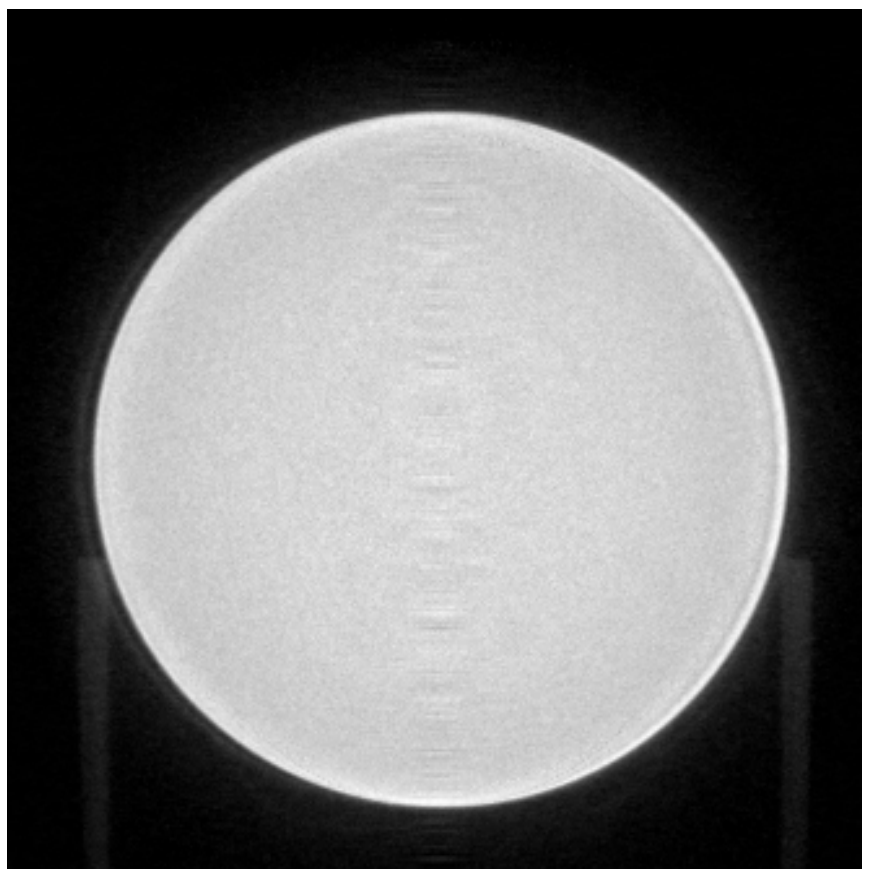

Y slice, average of slices 223-227. 
SN 6-92-004

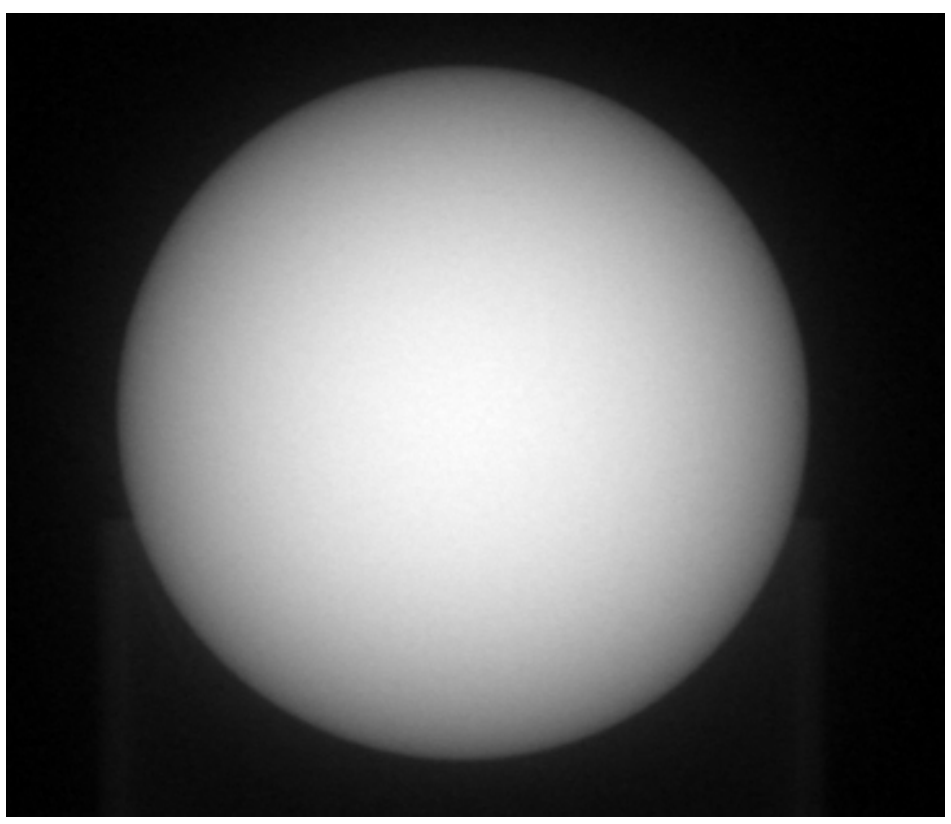

Attenuation radiograph, 0 degree.

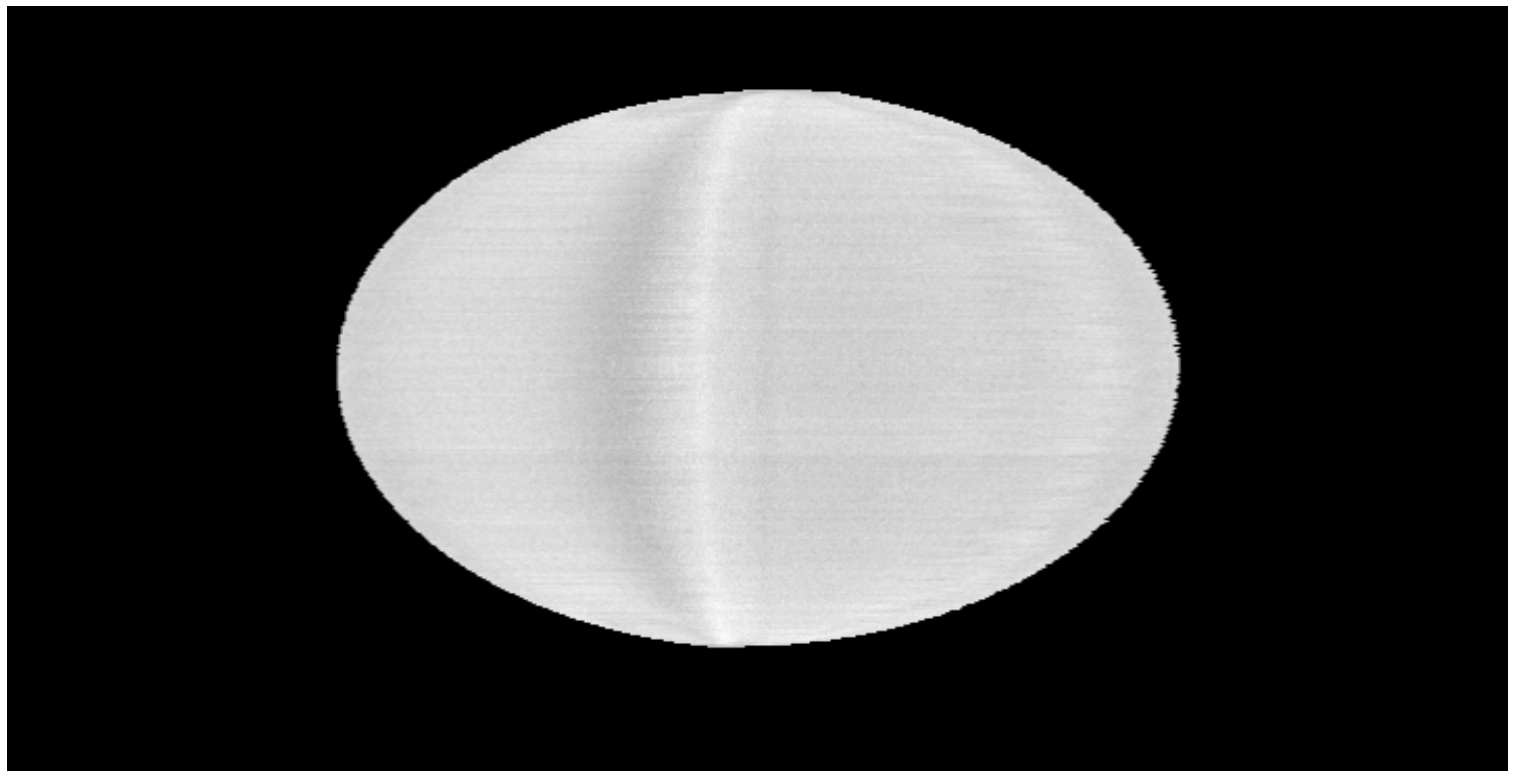

Shell extraction, 5 pixels inside of outer surface. 


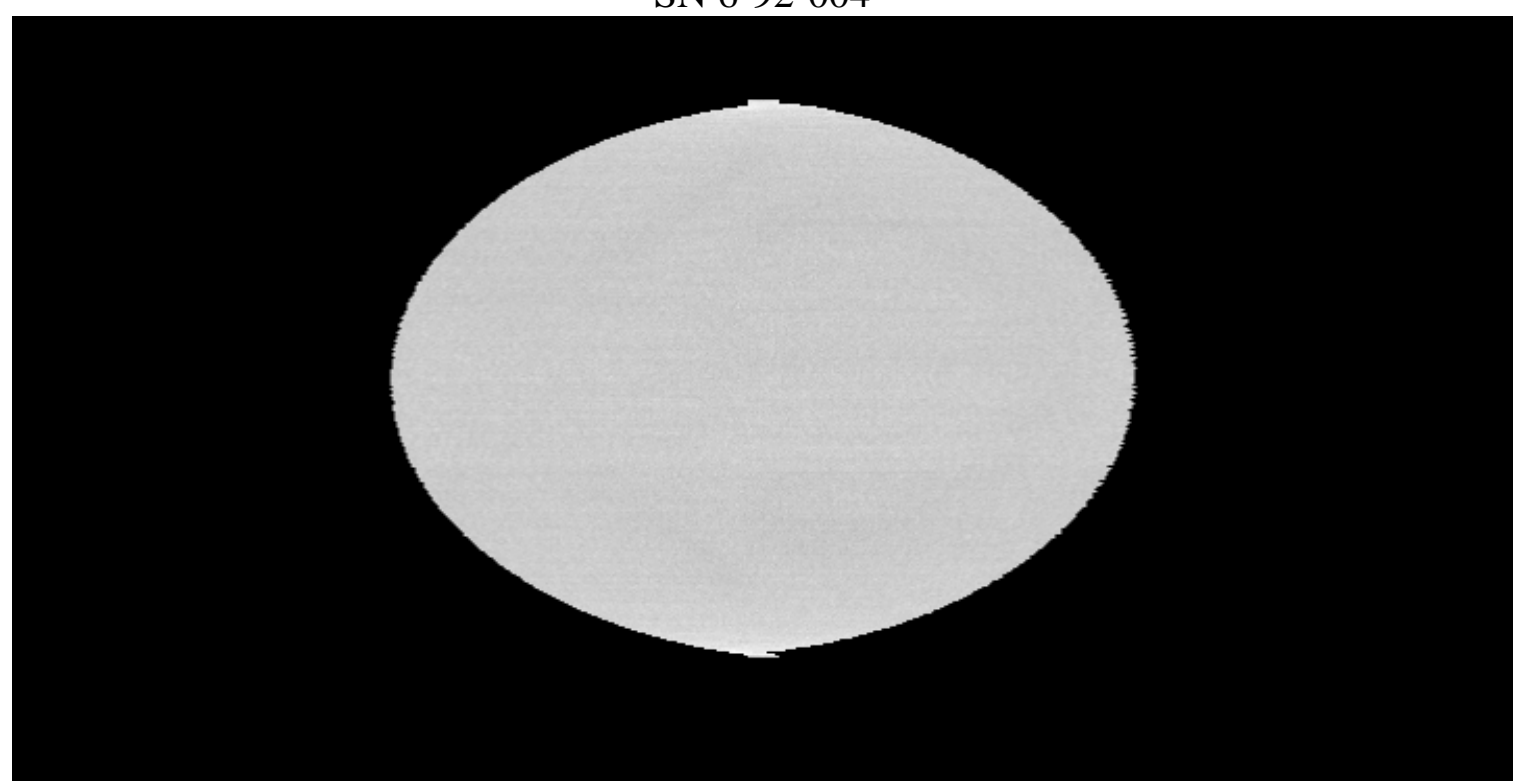

Shell extraction, 20 pixels inside of outer surface.

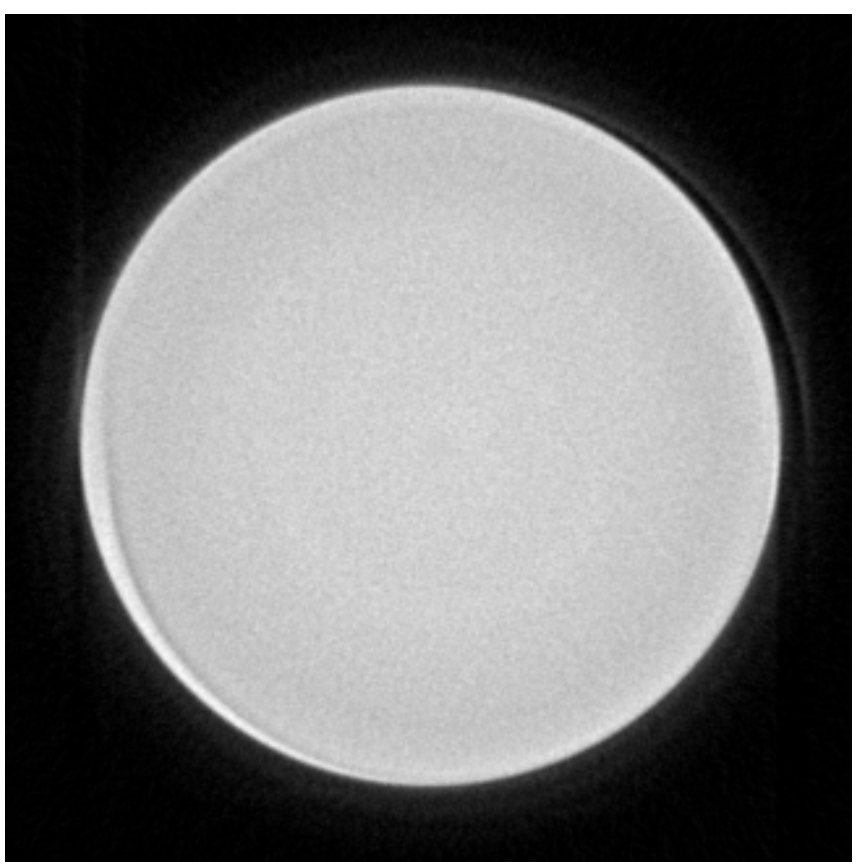

Z slice average of slices 223-227,

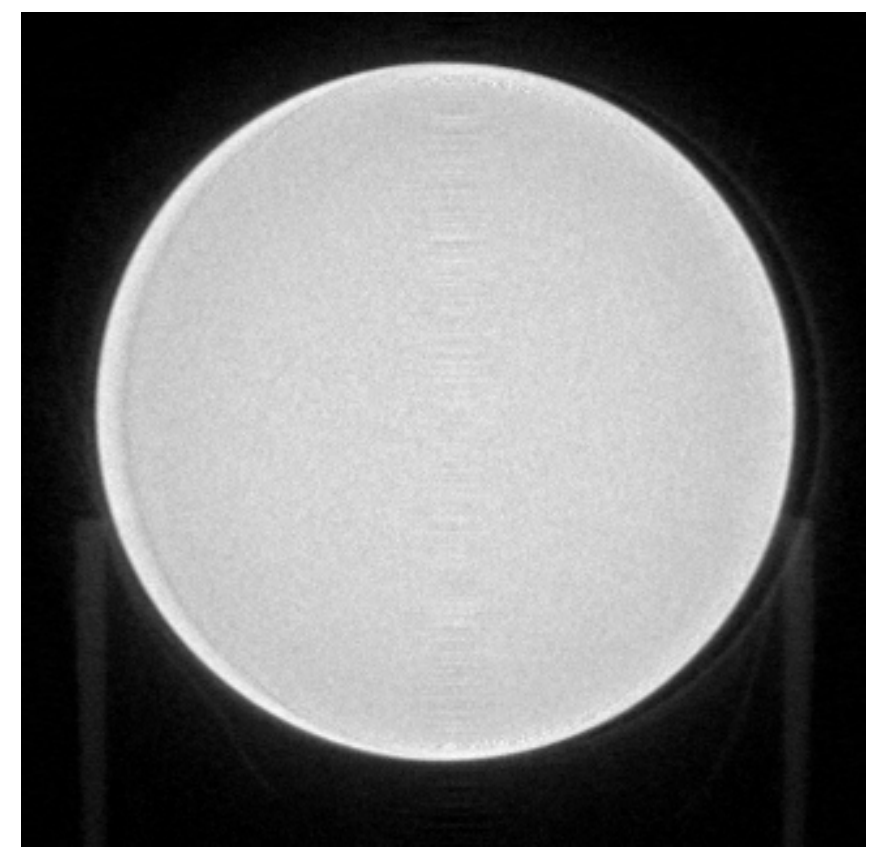

Y slice average of slices 223-227. 
SN 6-92-005

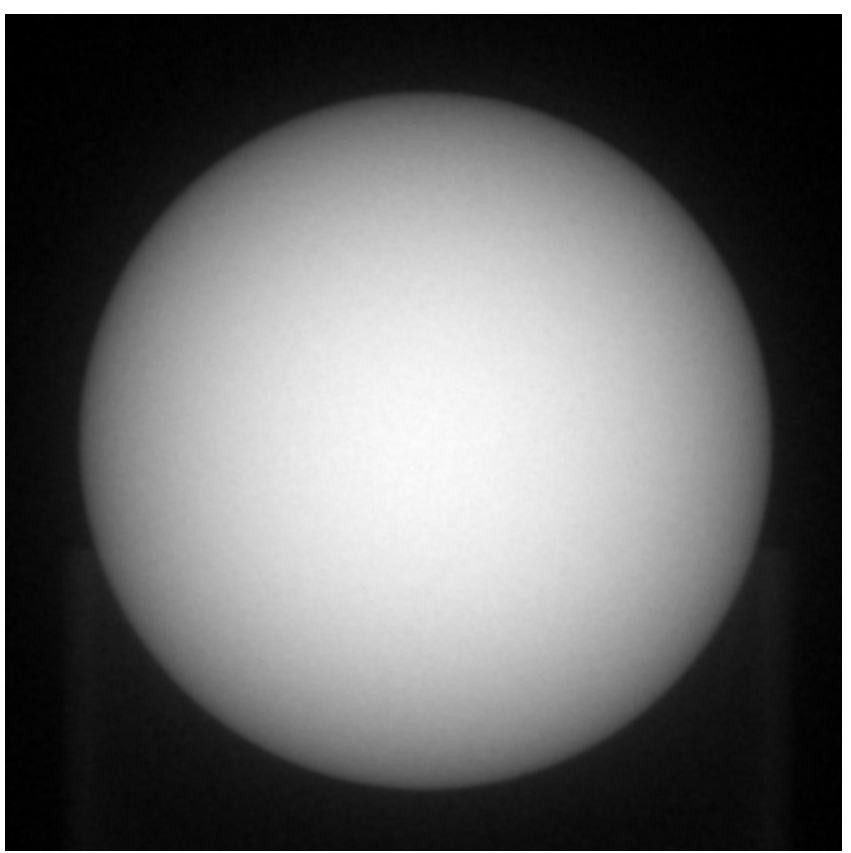

Attenuation radiograph, 0 degree.

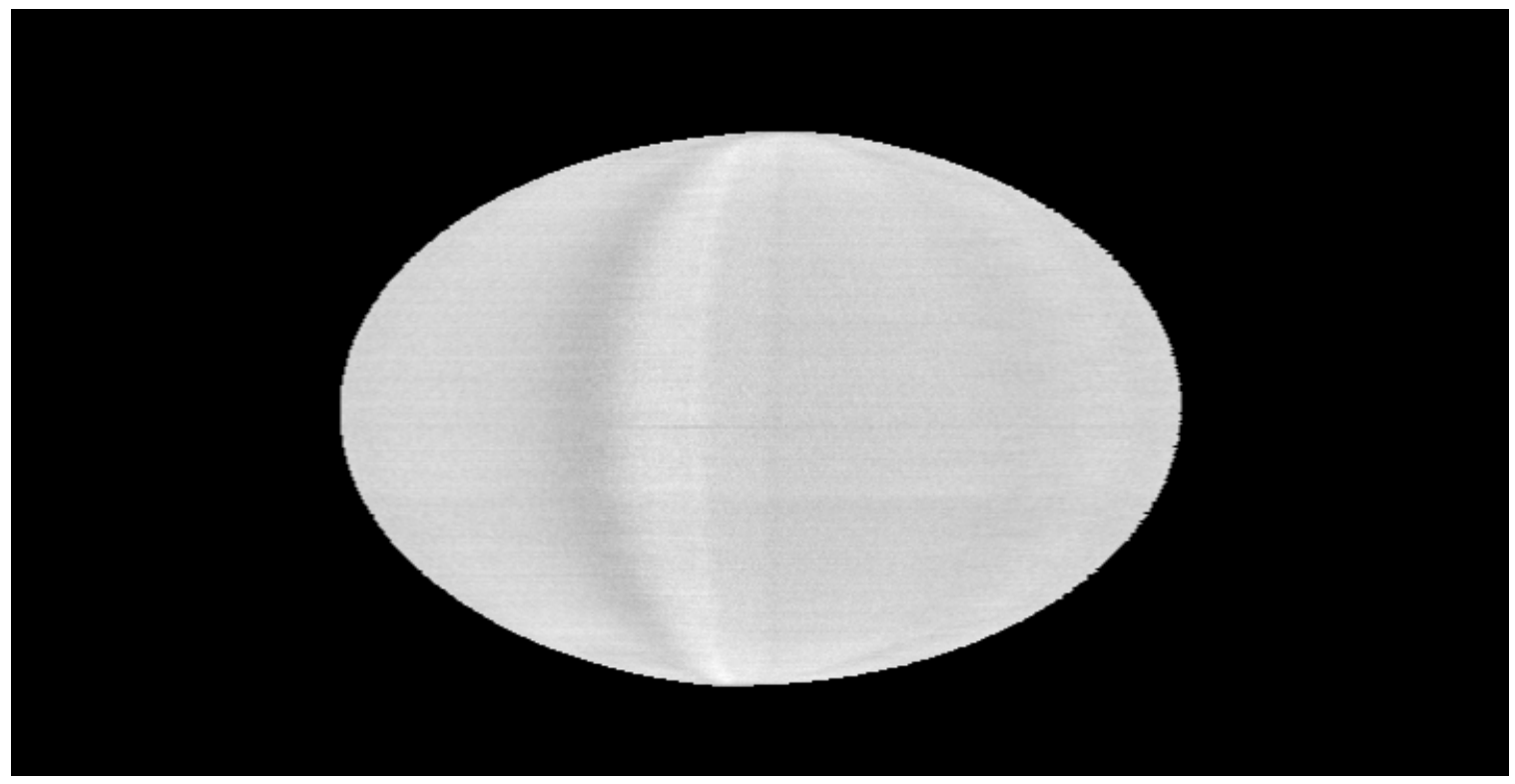

Shell extraction, 5 pixels inside of outer surface. 


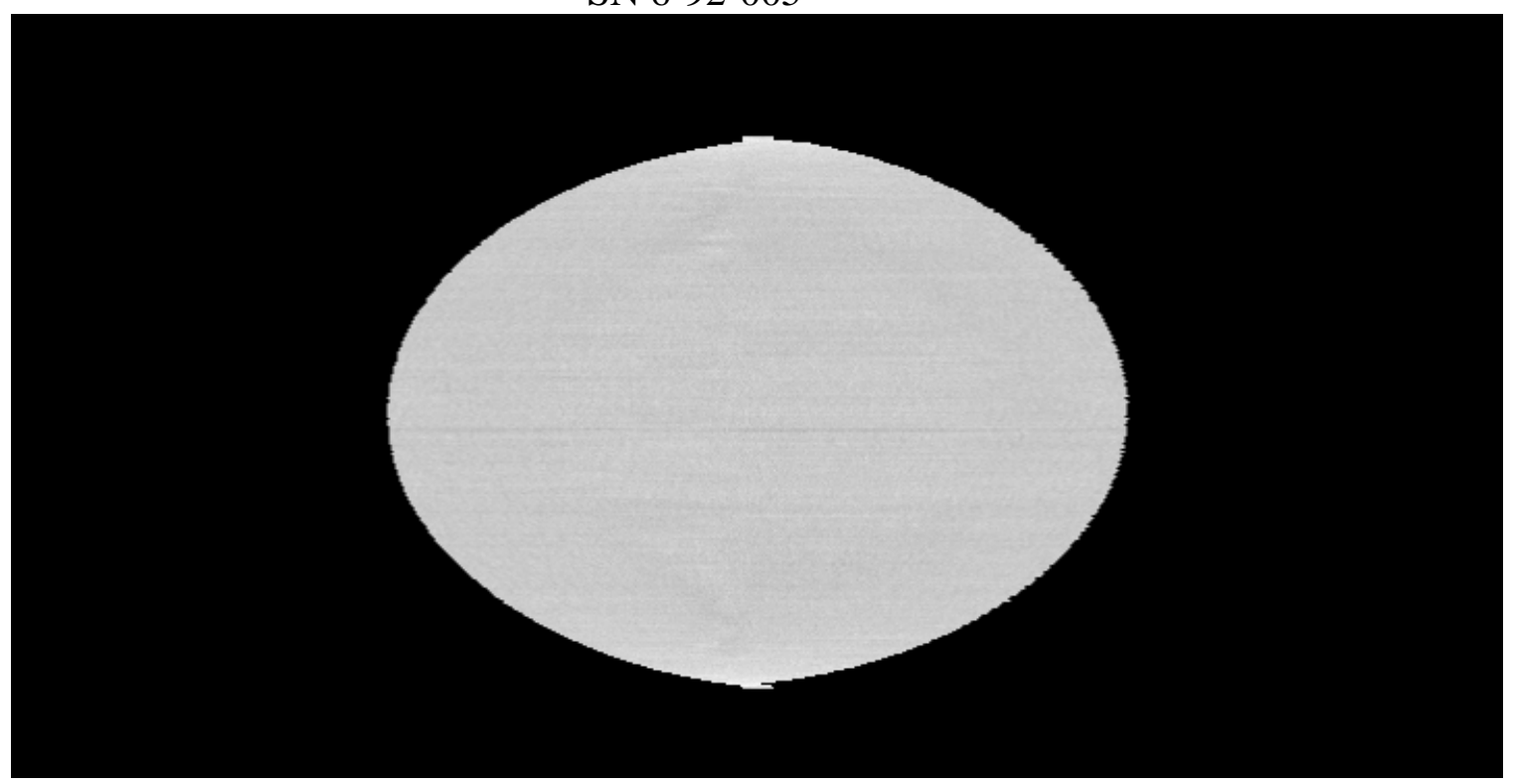

Shell extraction, taken 20 pixels inside of outer surface.
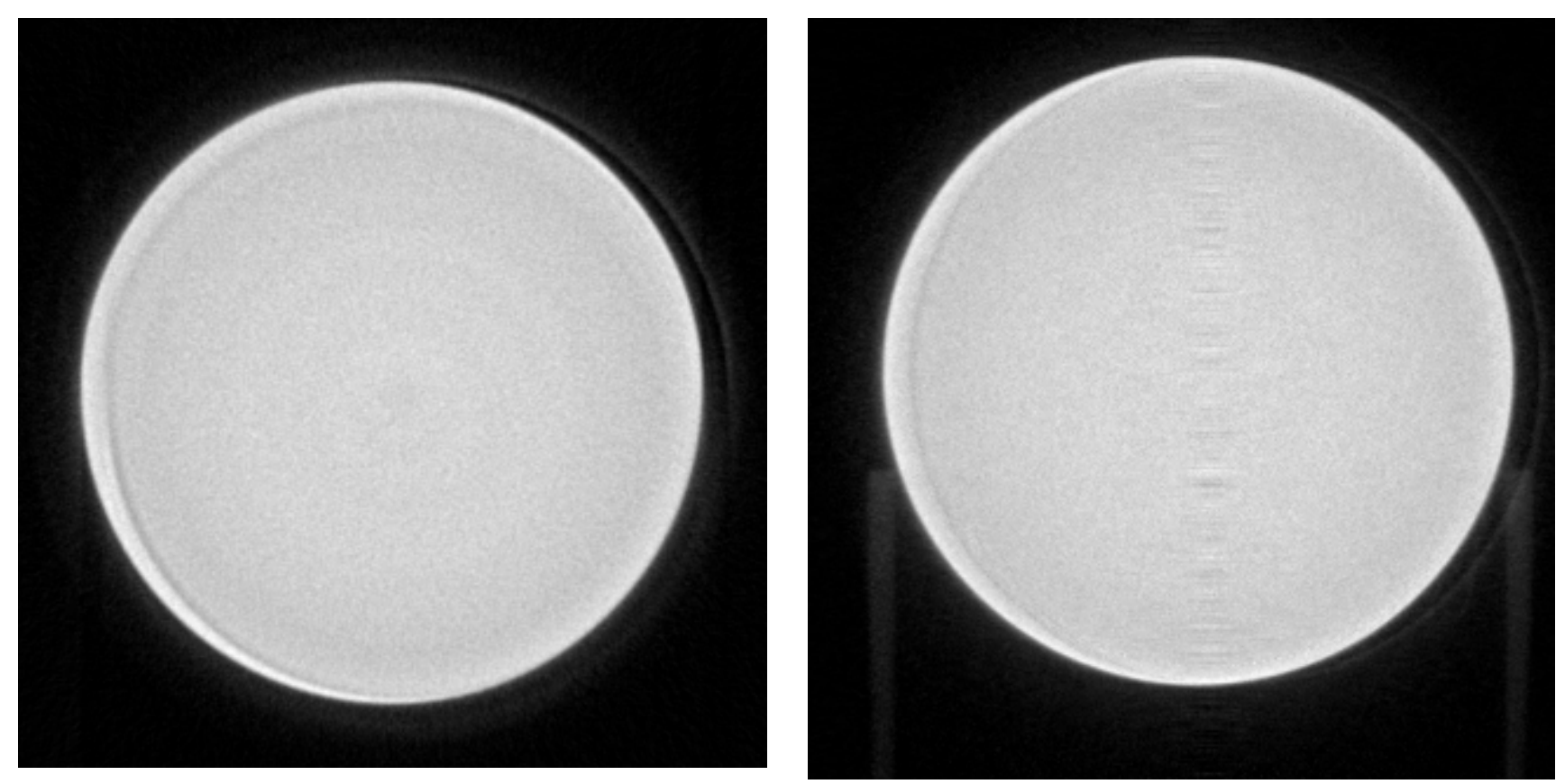

Z slice average of slices 223-227

Y slice average of slices 223-227. 


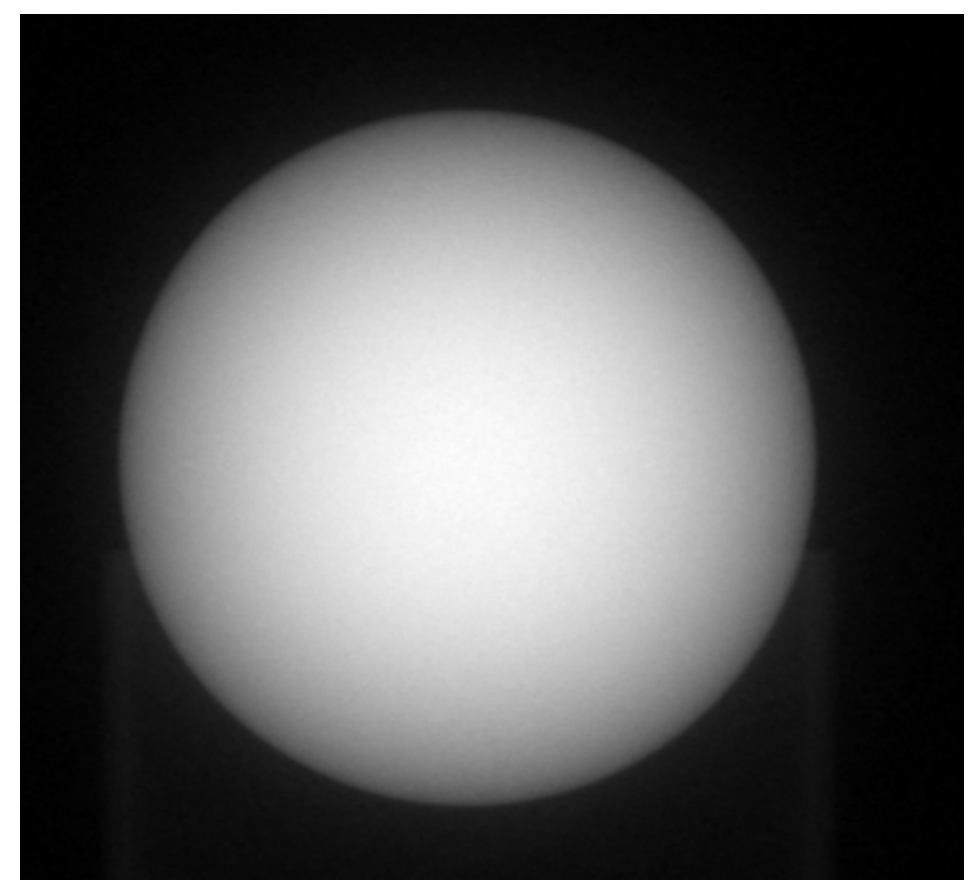

Attenuation radiograph, 0 degree.

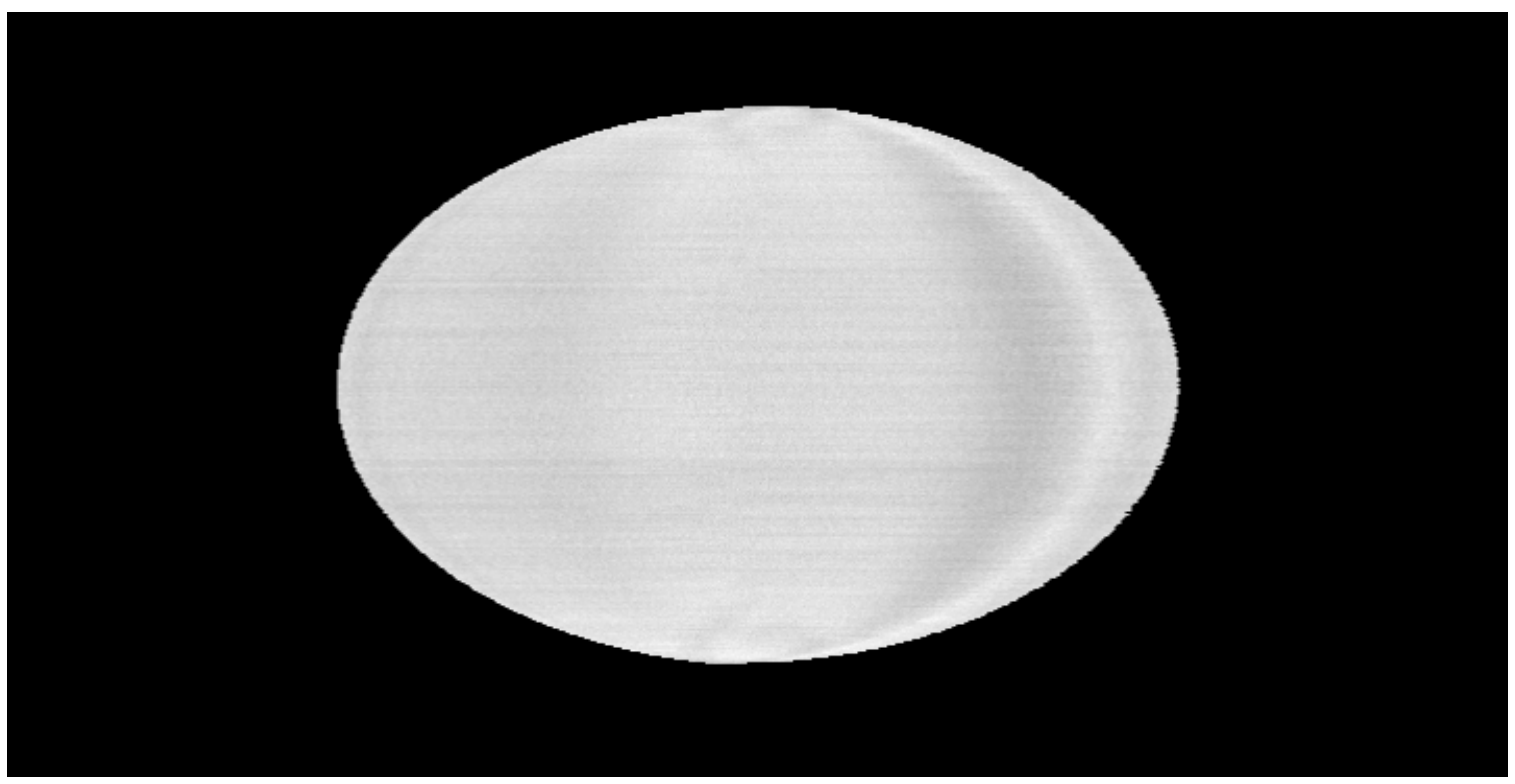

Shell extraction, 5 pixels inside of outer surface. 


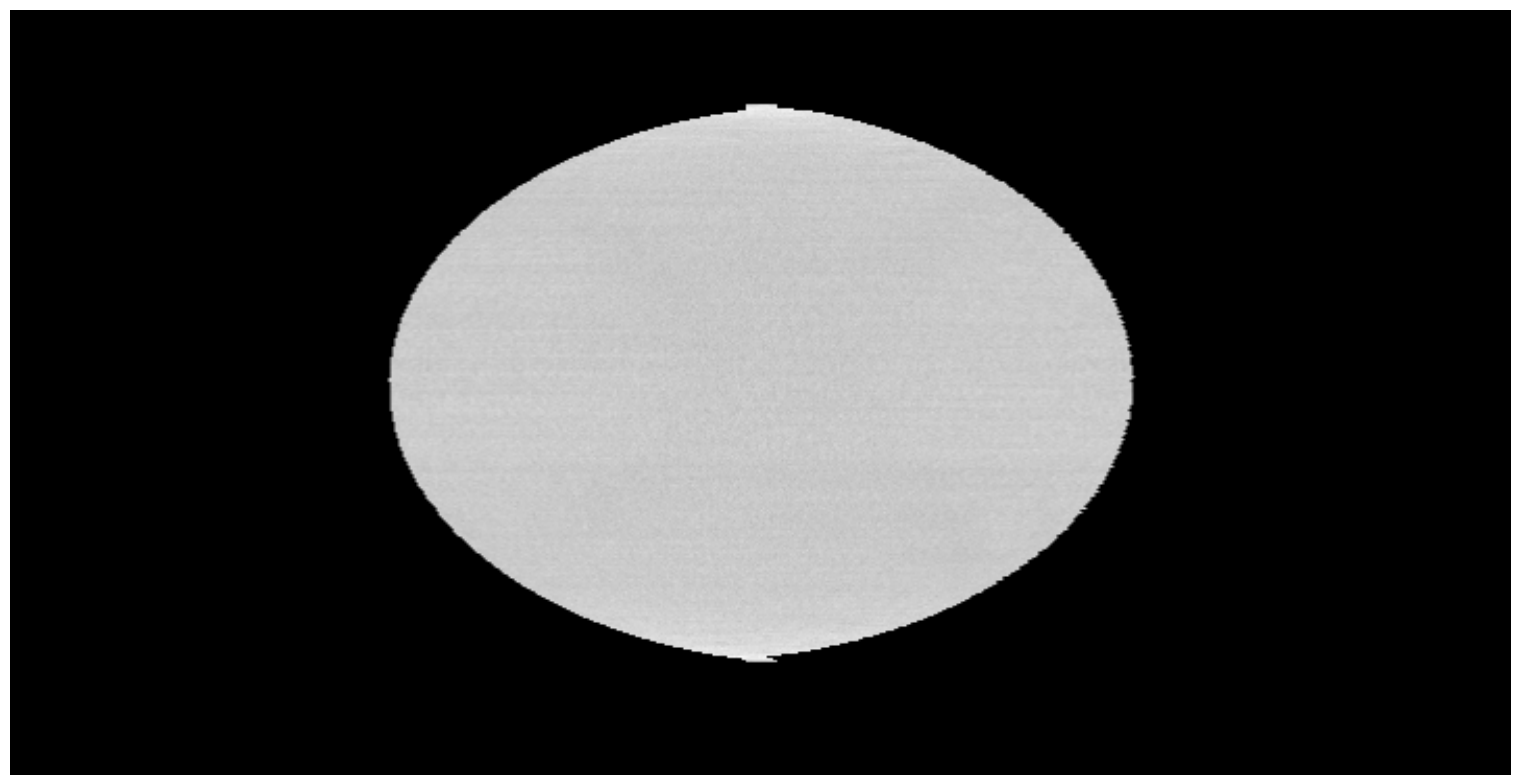

Shell extraction, taken 20 pixels inside of outer surface.

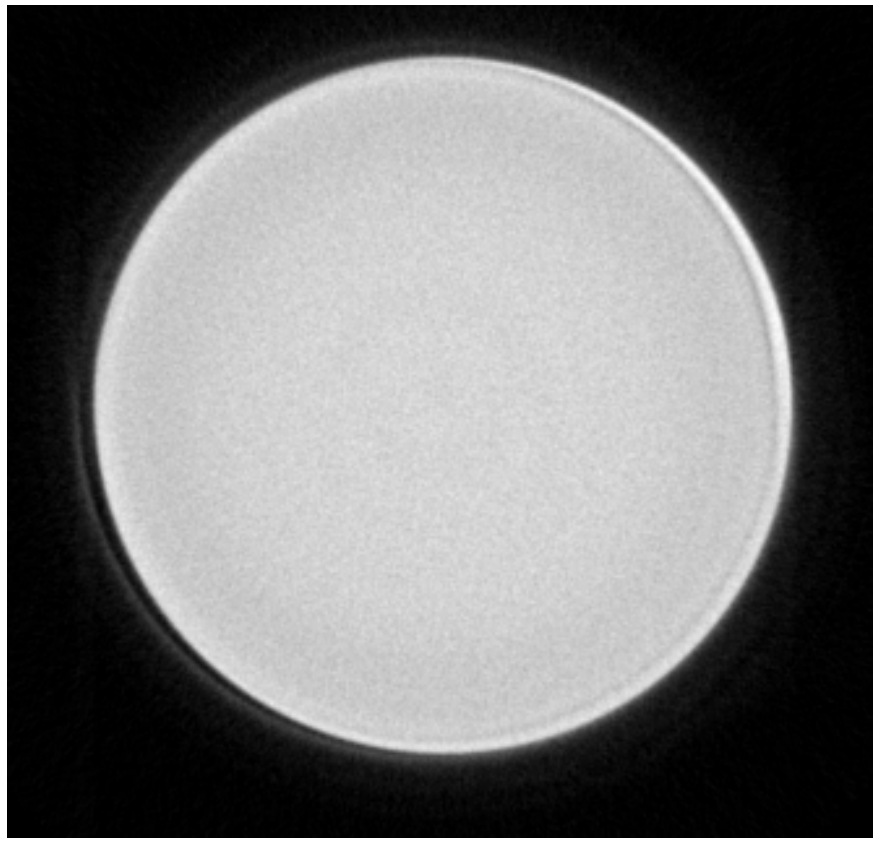

Z slice average of slices 223-227

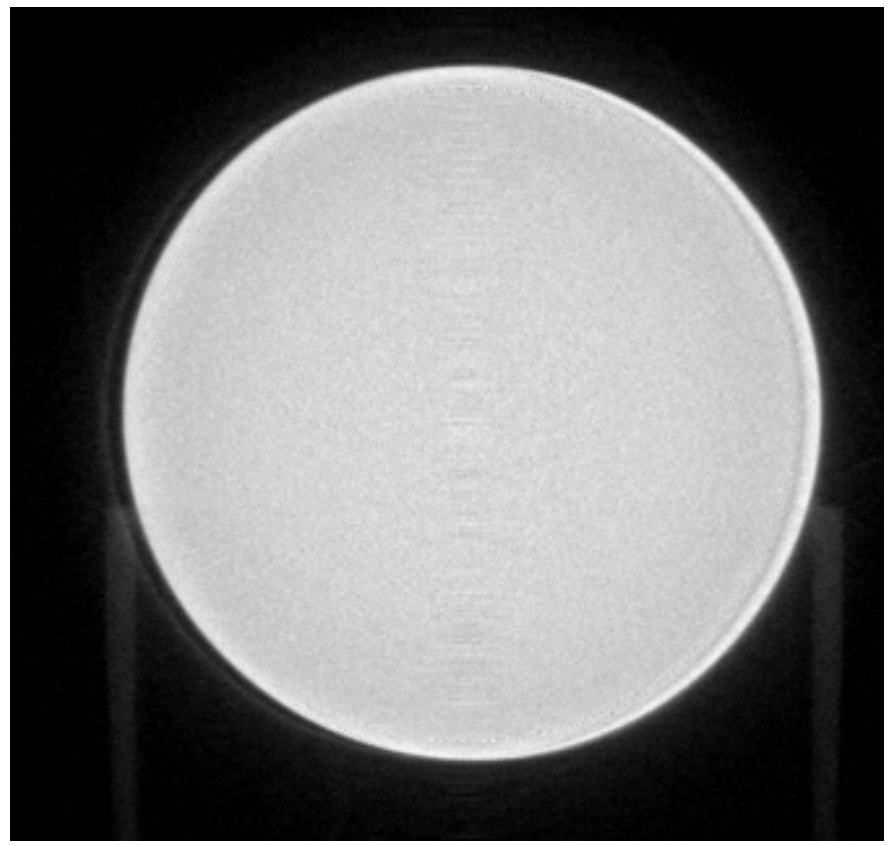

Y slice average of slices 223-227. 
Attenuation radiograph, 0 degree.

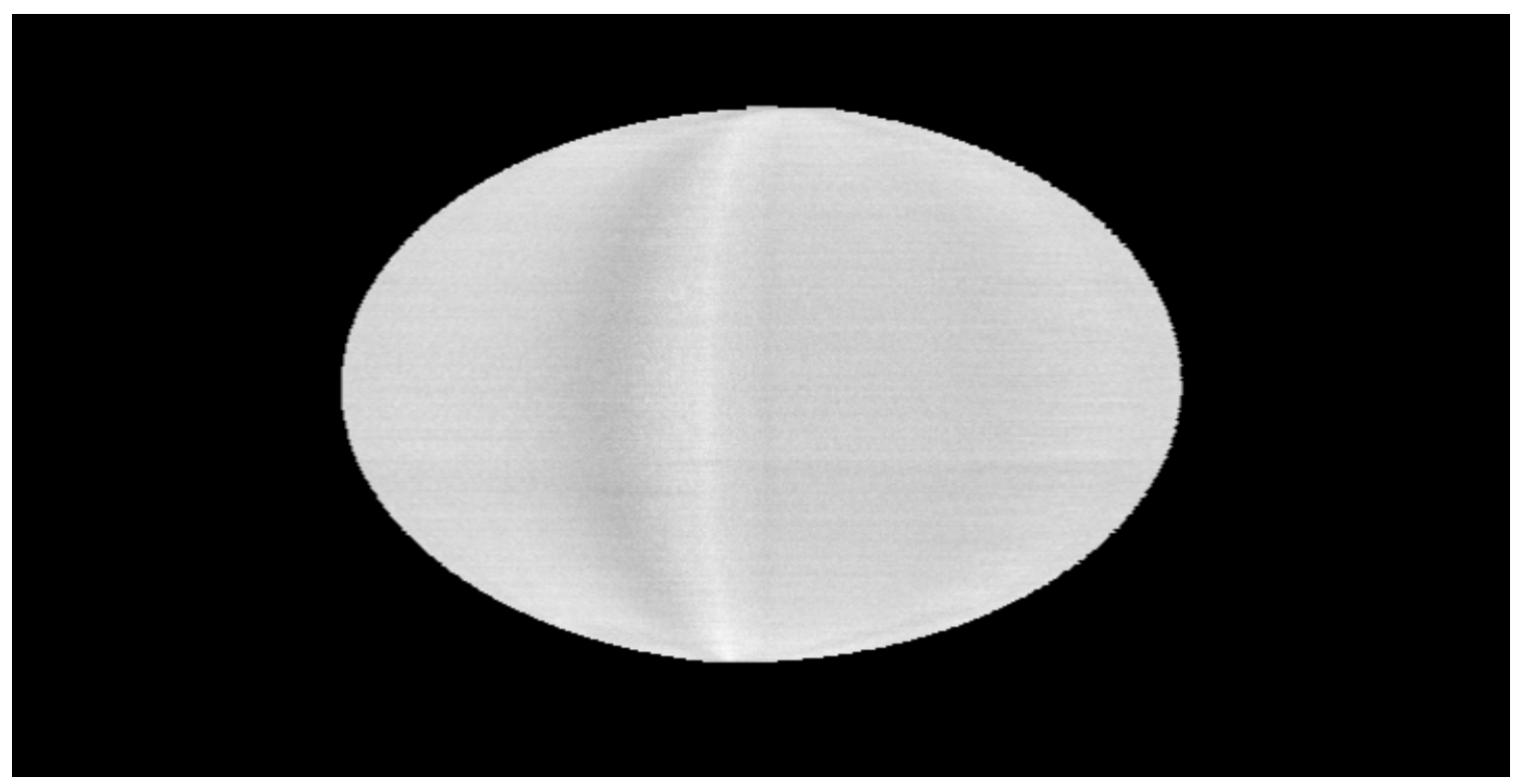

Shell extraction, 5 pixels inside of outer surface. 
SN 6-92-007

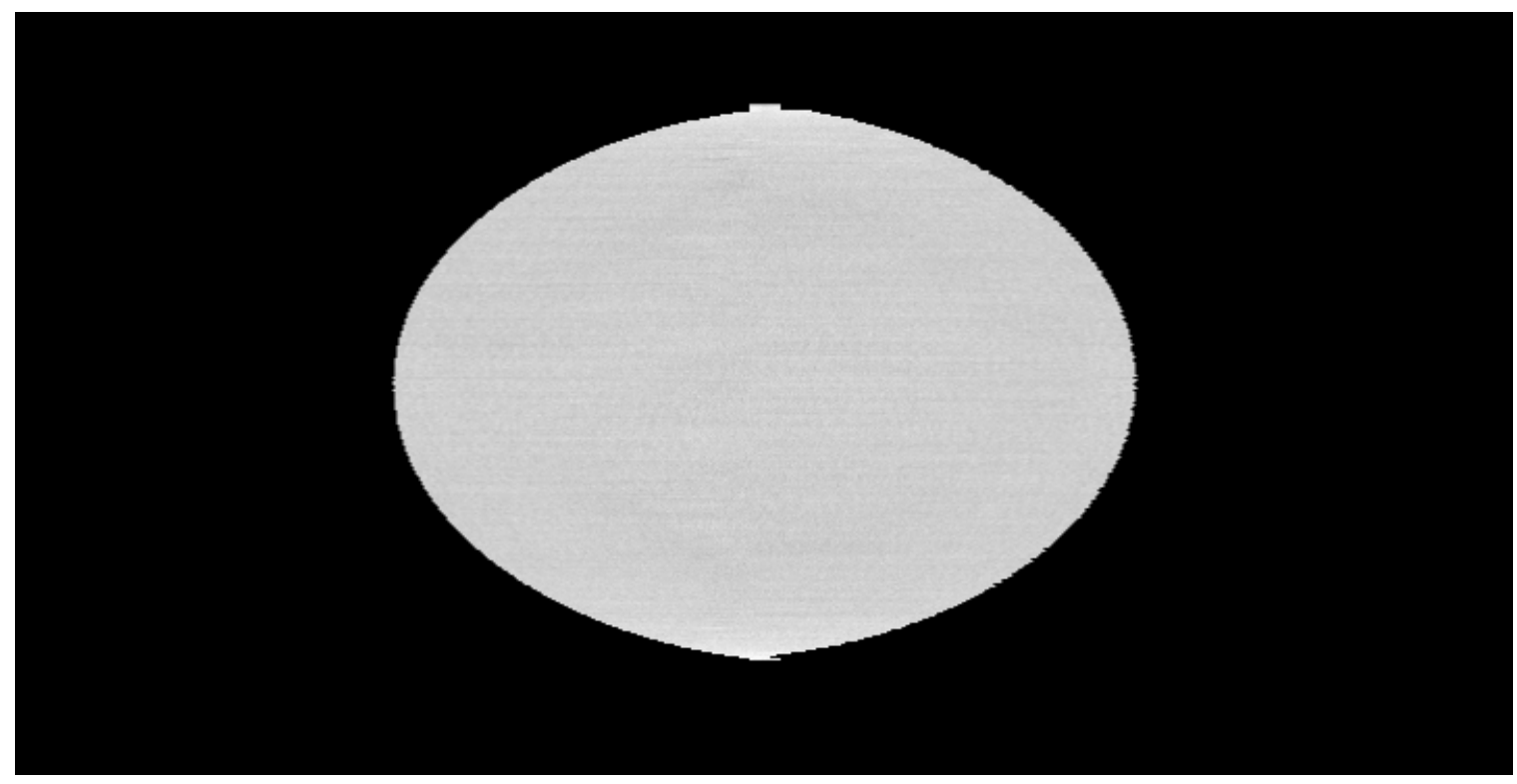

Shell extraction, taken 20 pixels inside of outer surface.

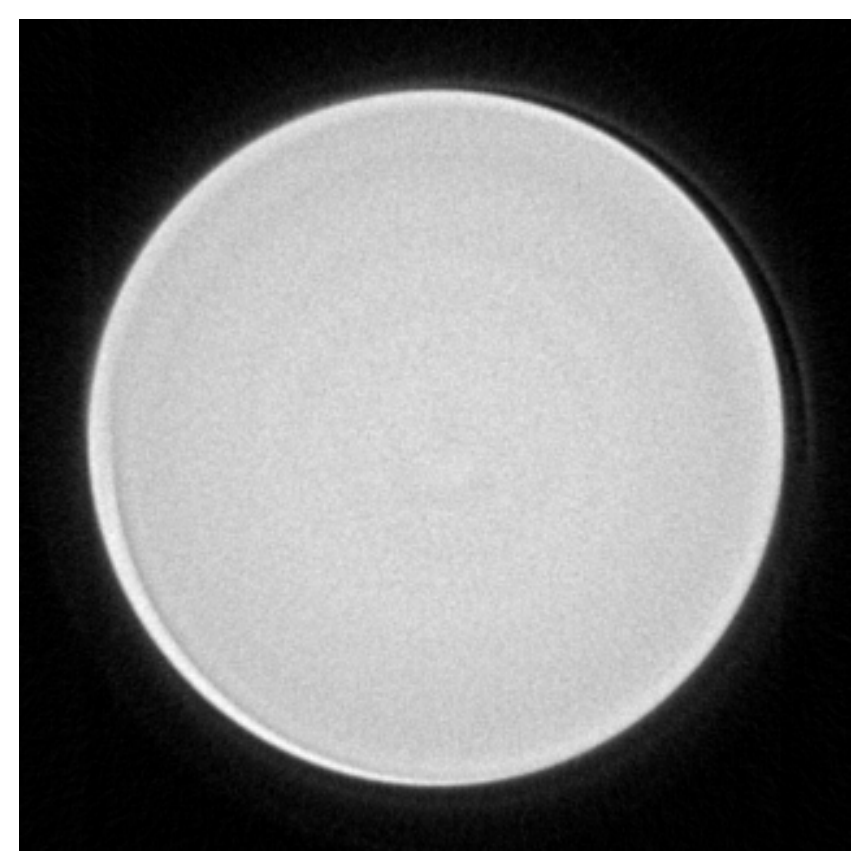

Z slice average of slices 223-227

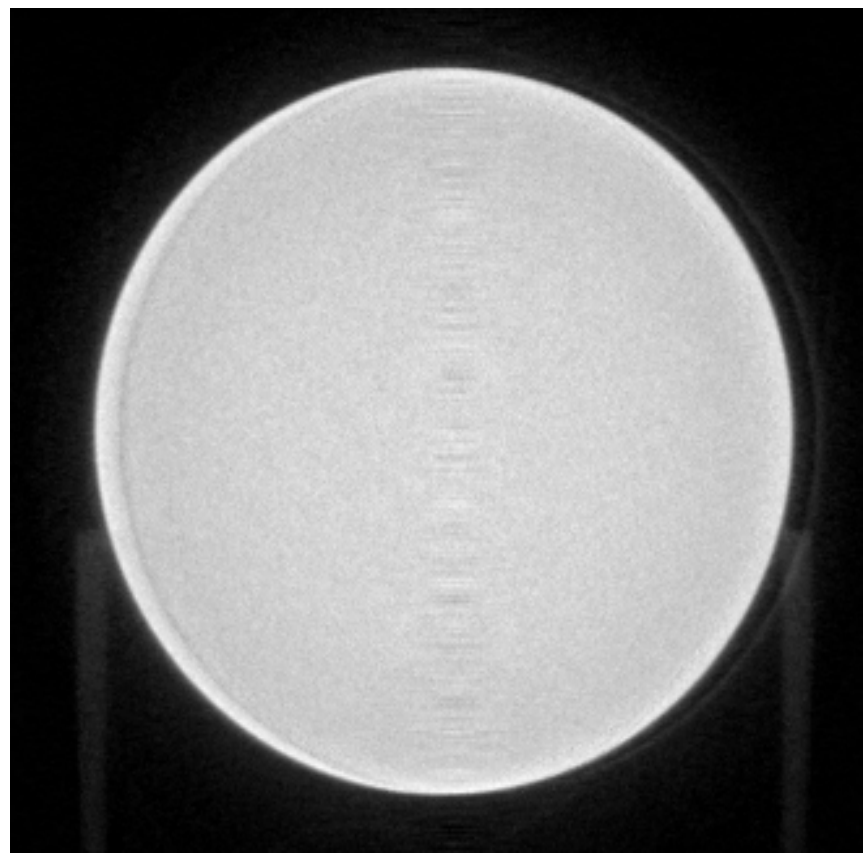

Y slice average of slices 223-227. 


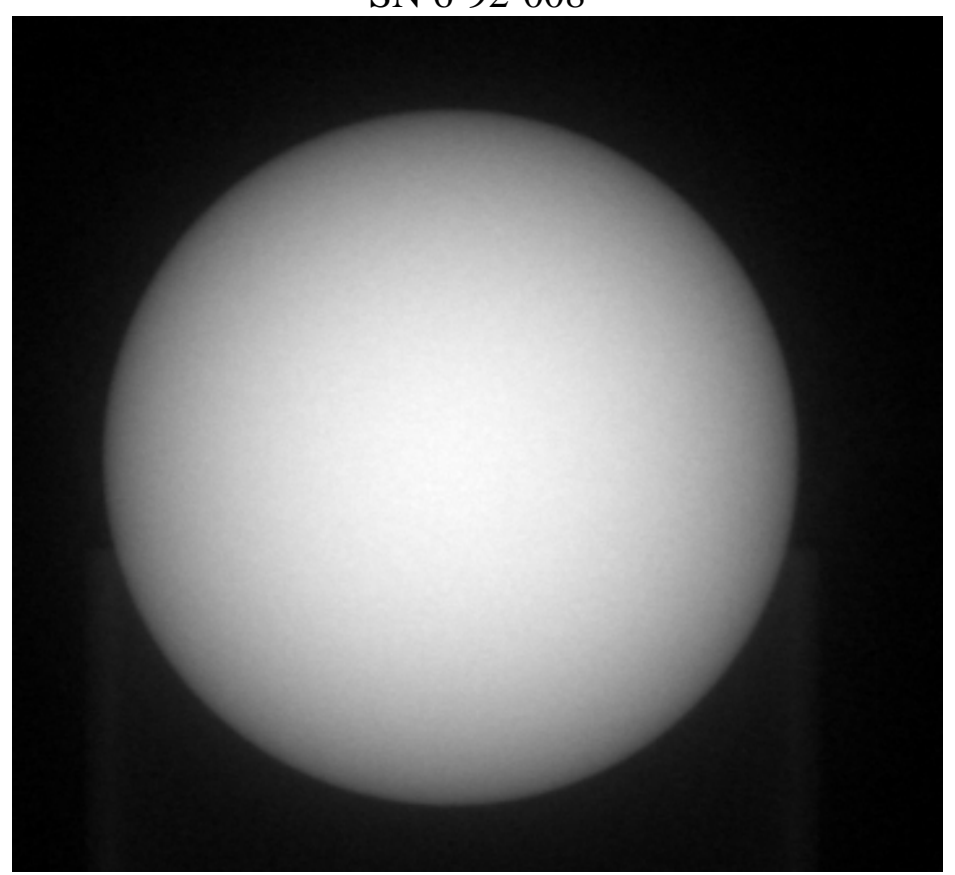

Attenuation radiograph, 0 degree.

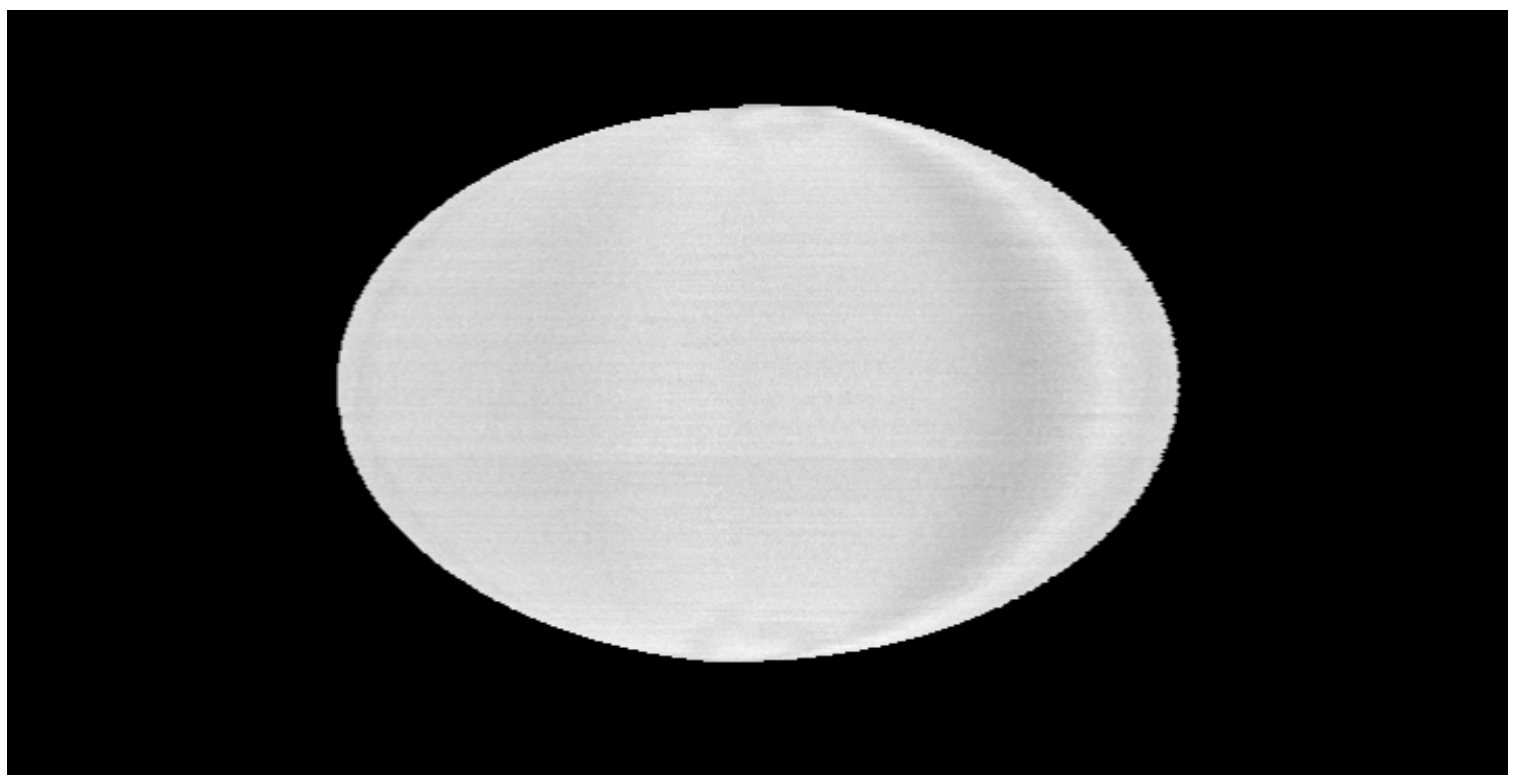

Shell extraction, 5 pixels inside of outer surface. 
SN 6-92-008

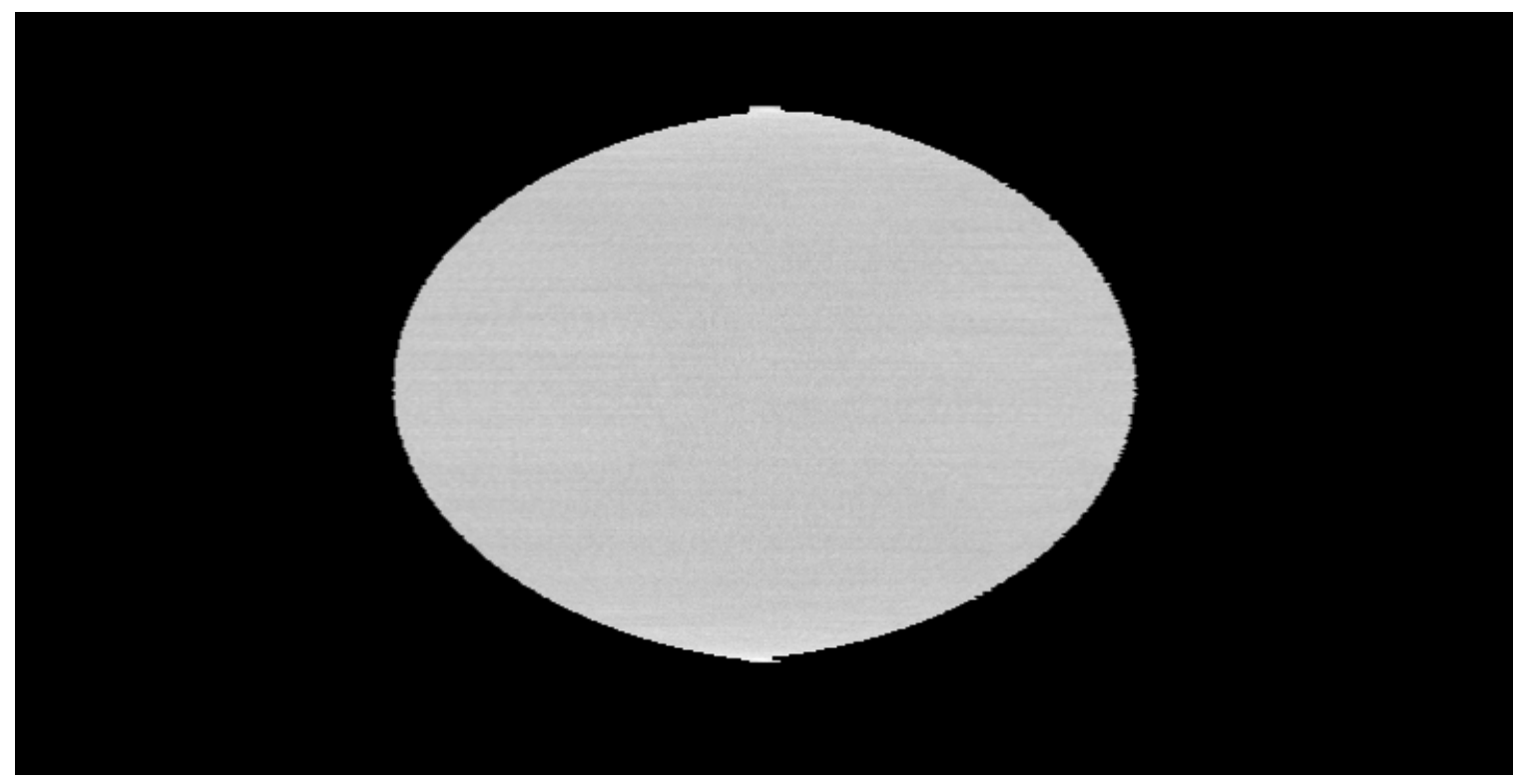

Shell extraction, taken 20 pixels inside of outer surface.

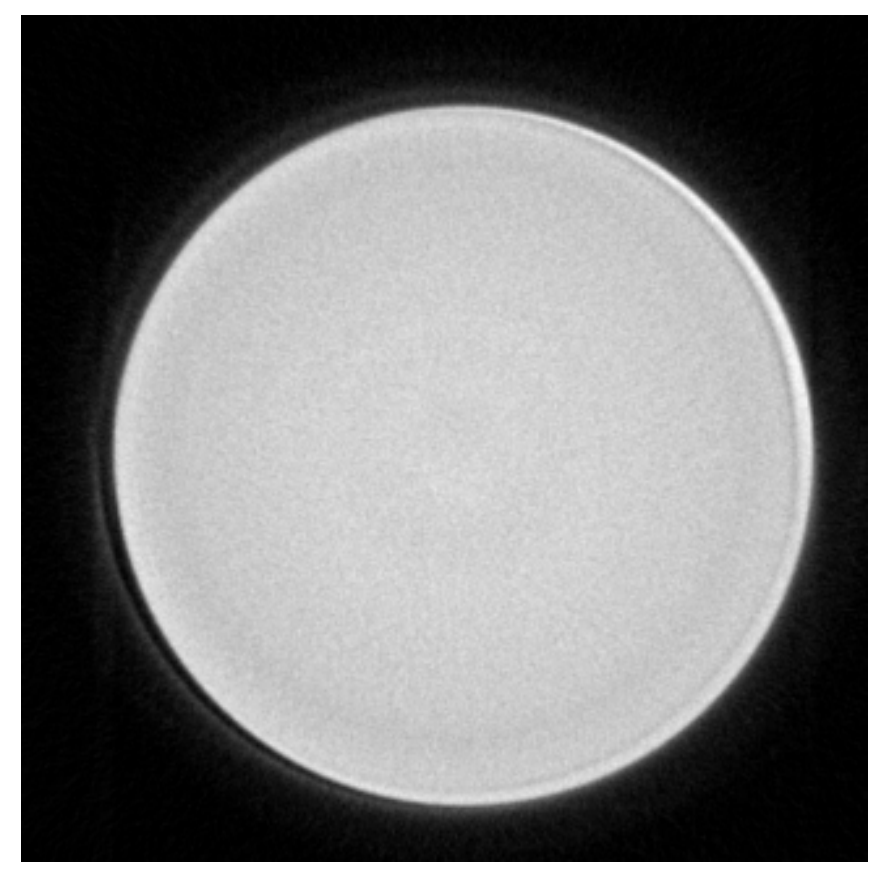

Z slice average of slices $223-227$

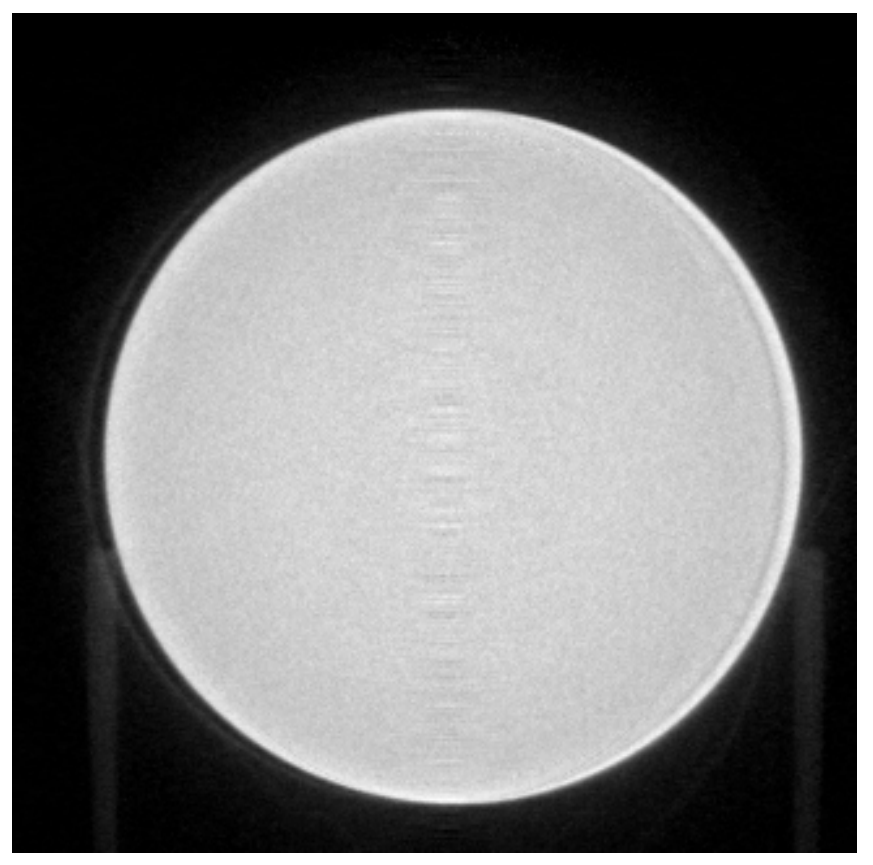

Y slice average of slices 223-227. 
SN 6-92-009

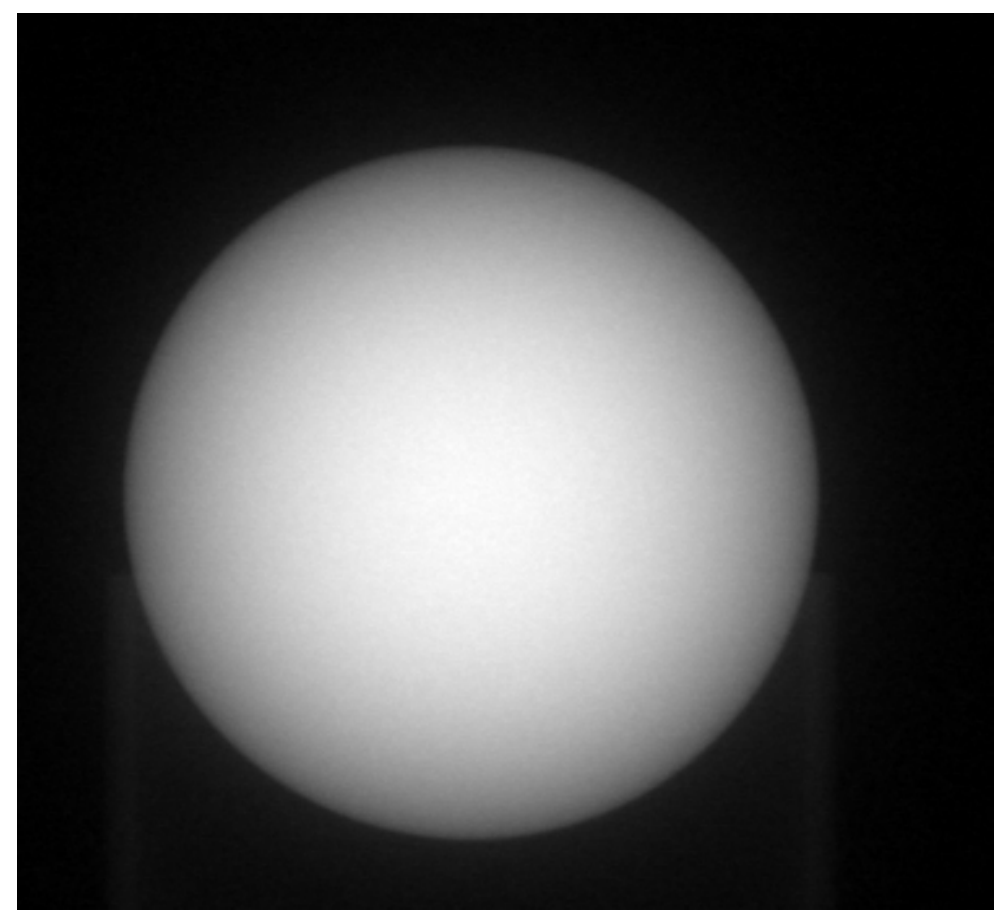

Attenuation radiograph, 0 degree.

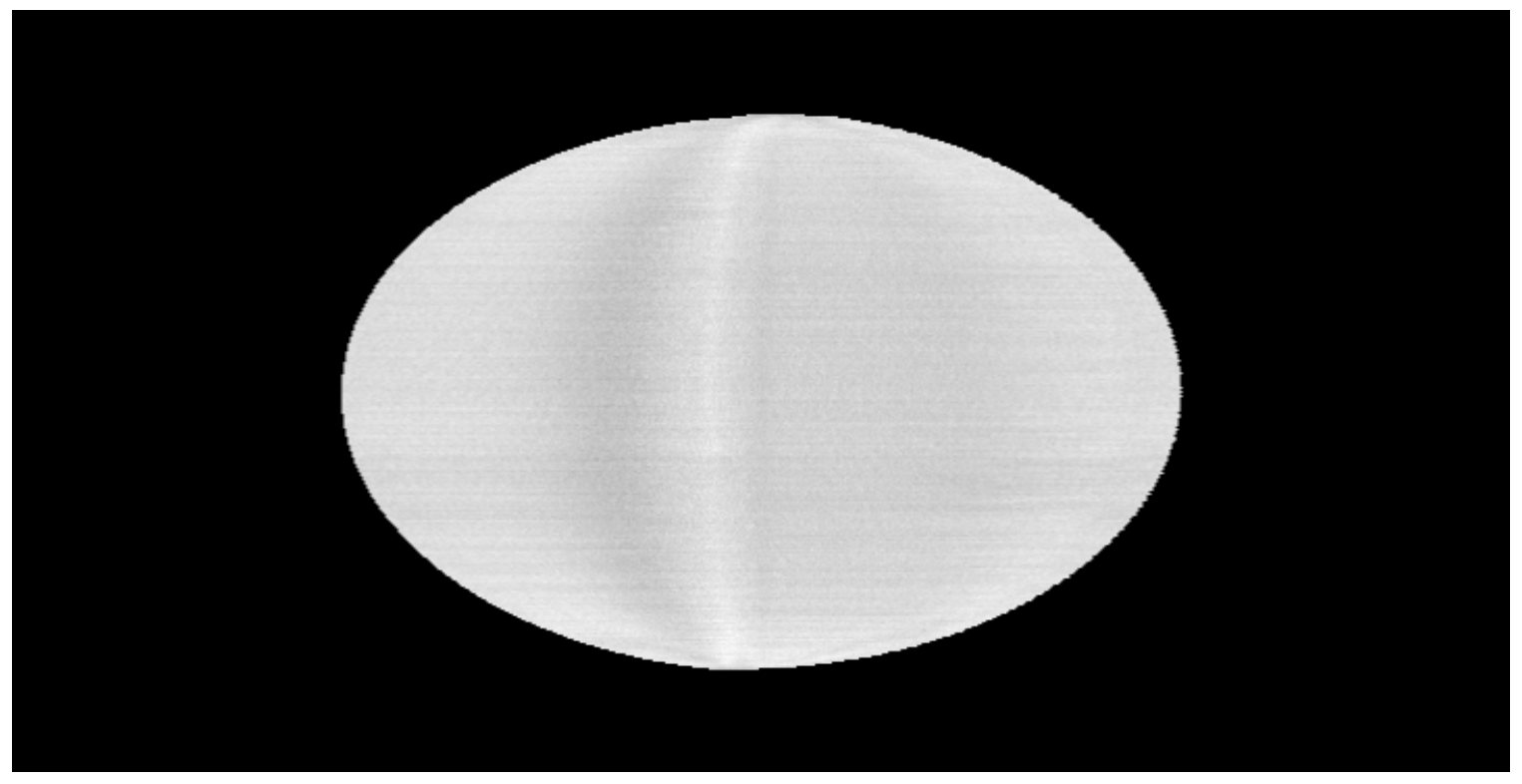

Shell extraction, 5 pixels inside of outer surface. 
SN 6-92-009

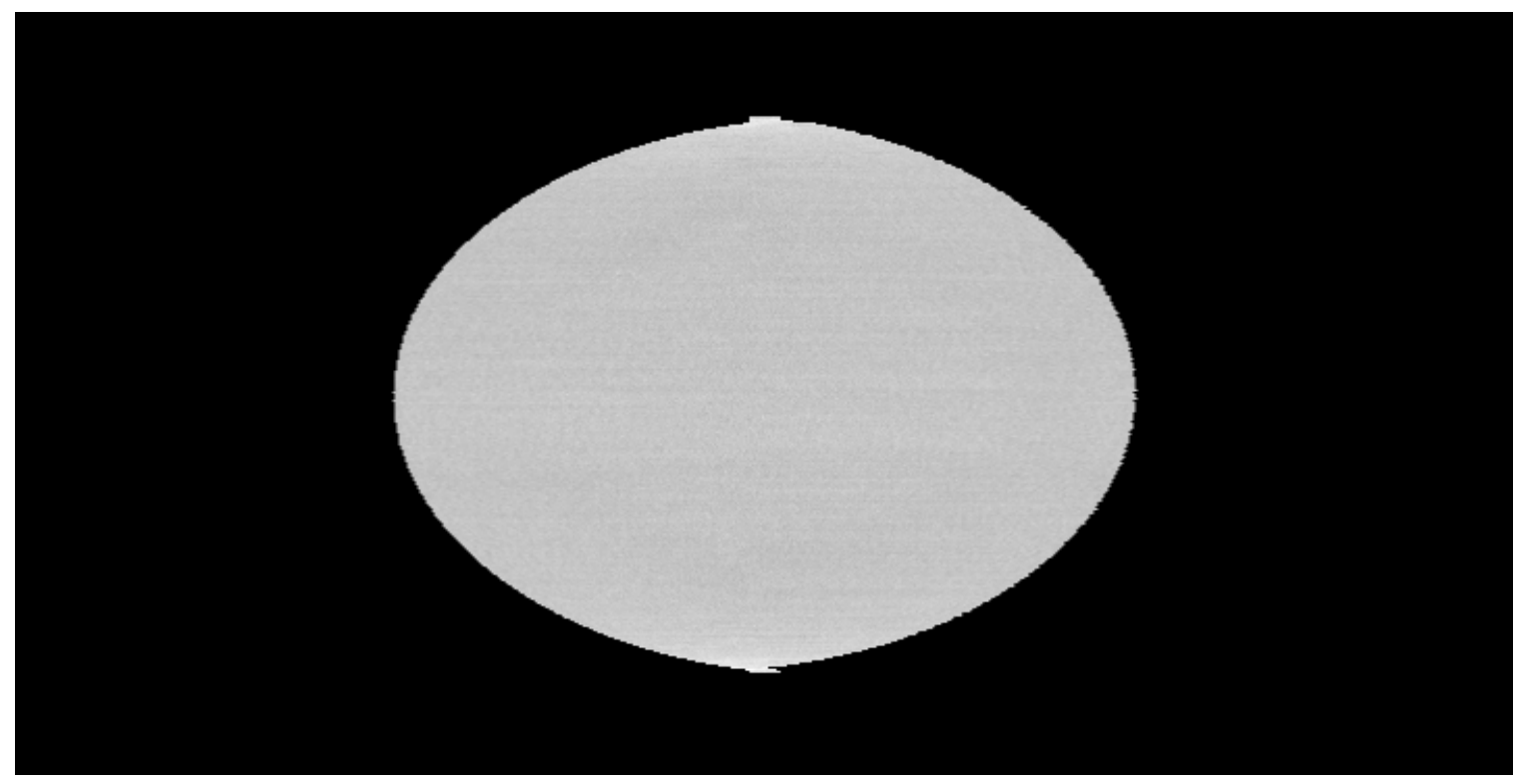

Shell extraction, taken 20 pixels inside of outer surface.

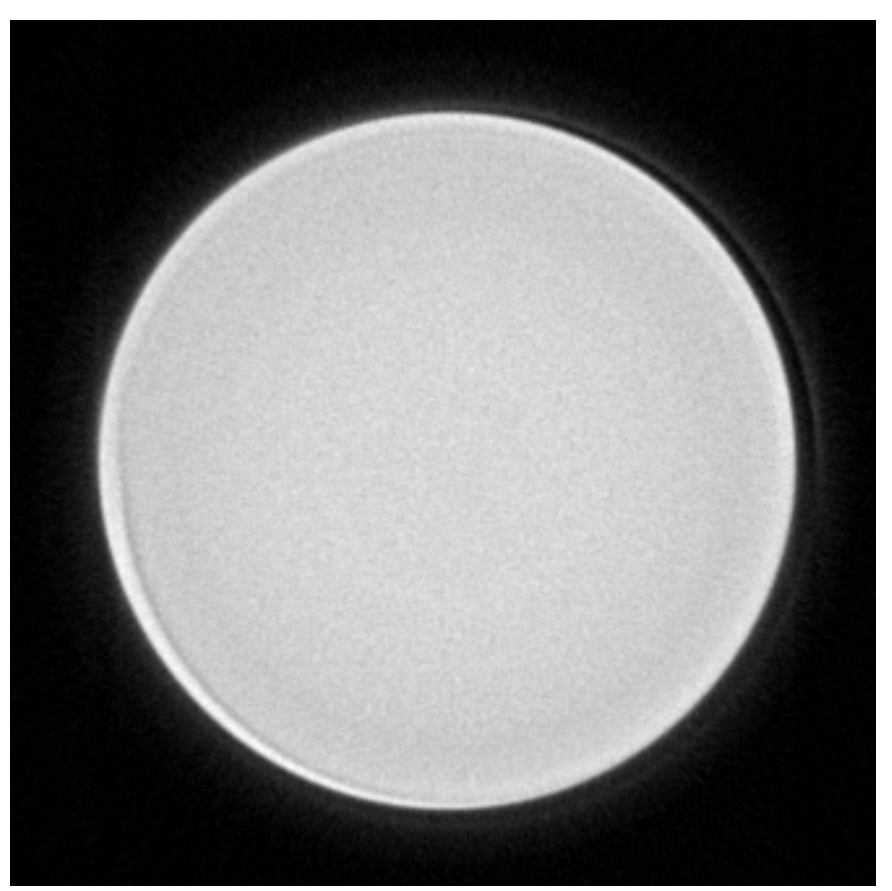

Z slice average of slices 223-227

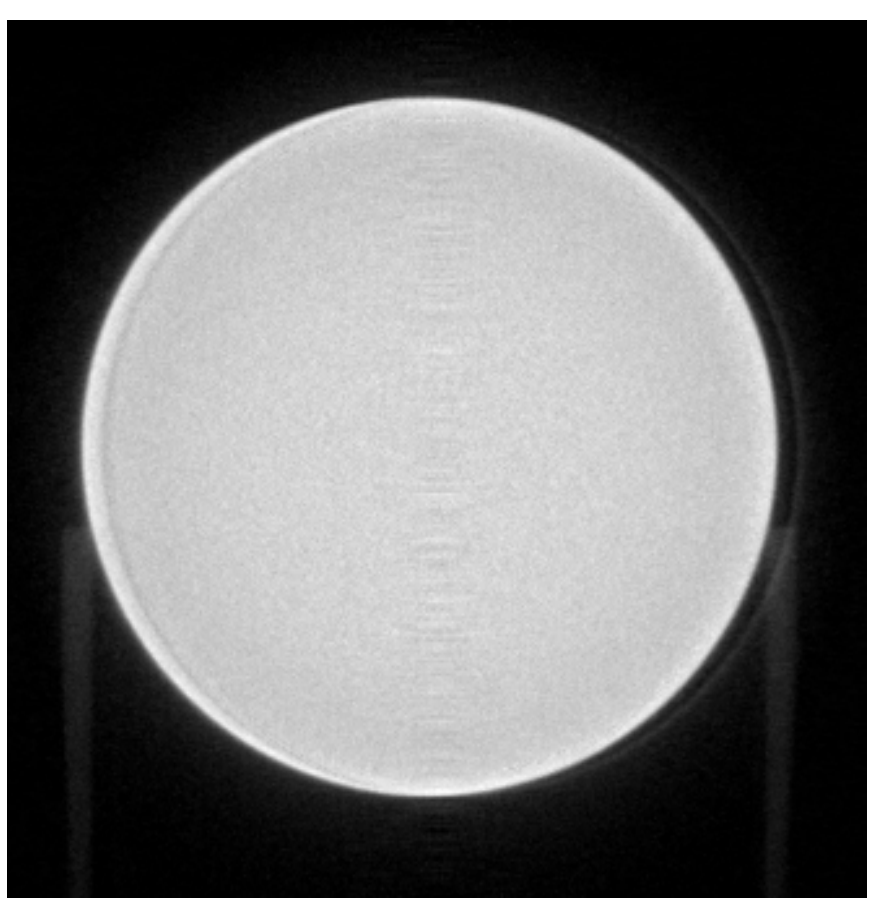

Y slice average of slices 223-227. 
SN 6-92-010

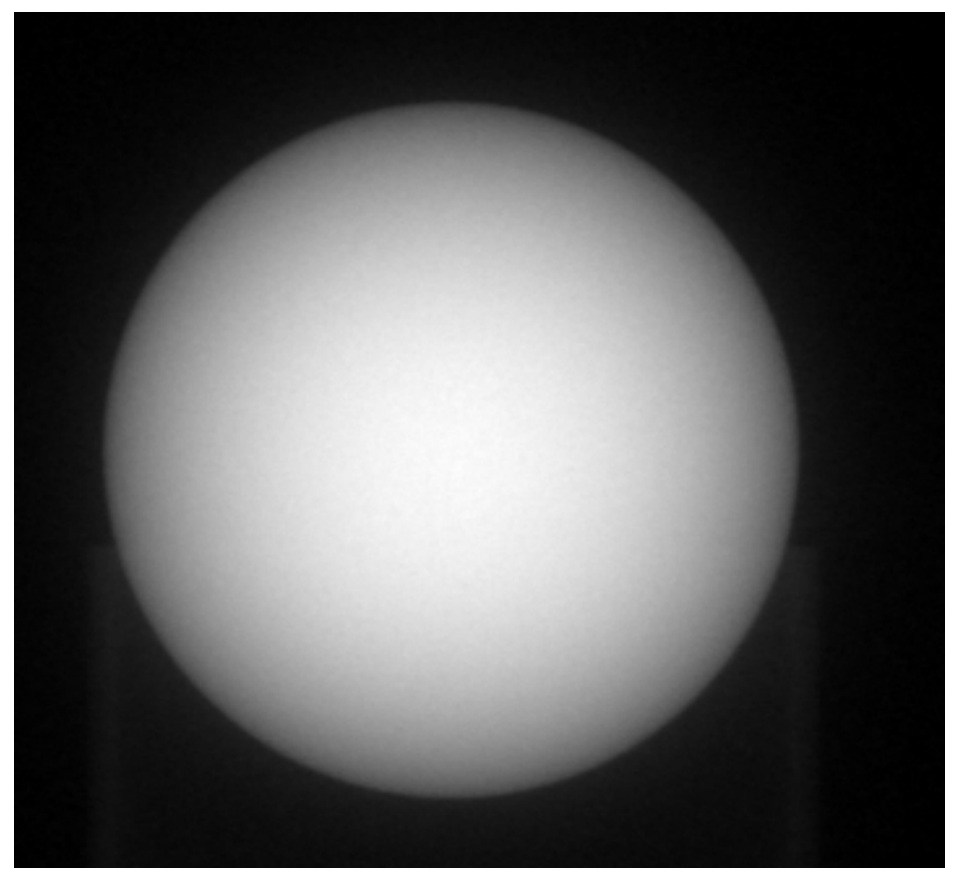

Attenuation radiograph, 0 degree.

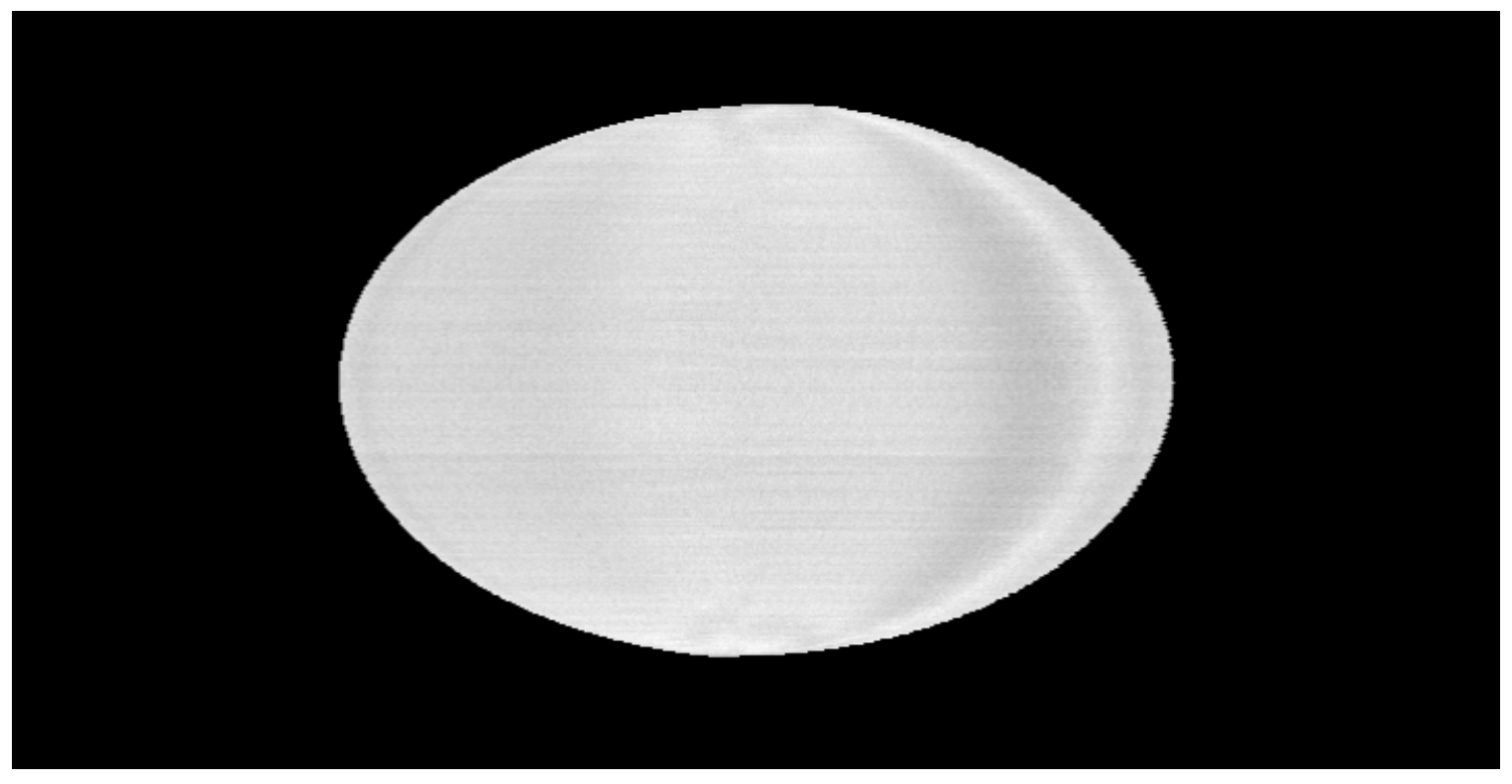

Shell extraction, 5 pixels inside of outer surface. 
SN 6-92-010

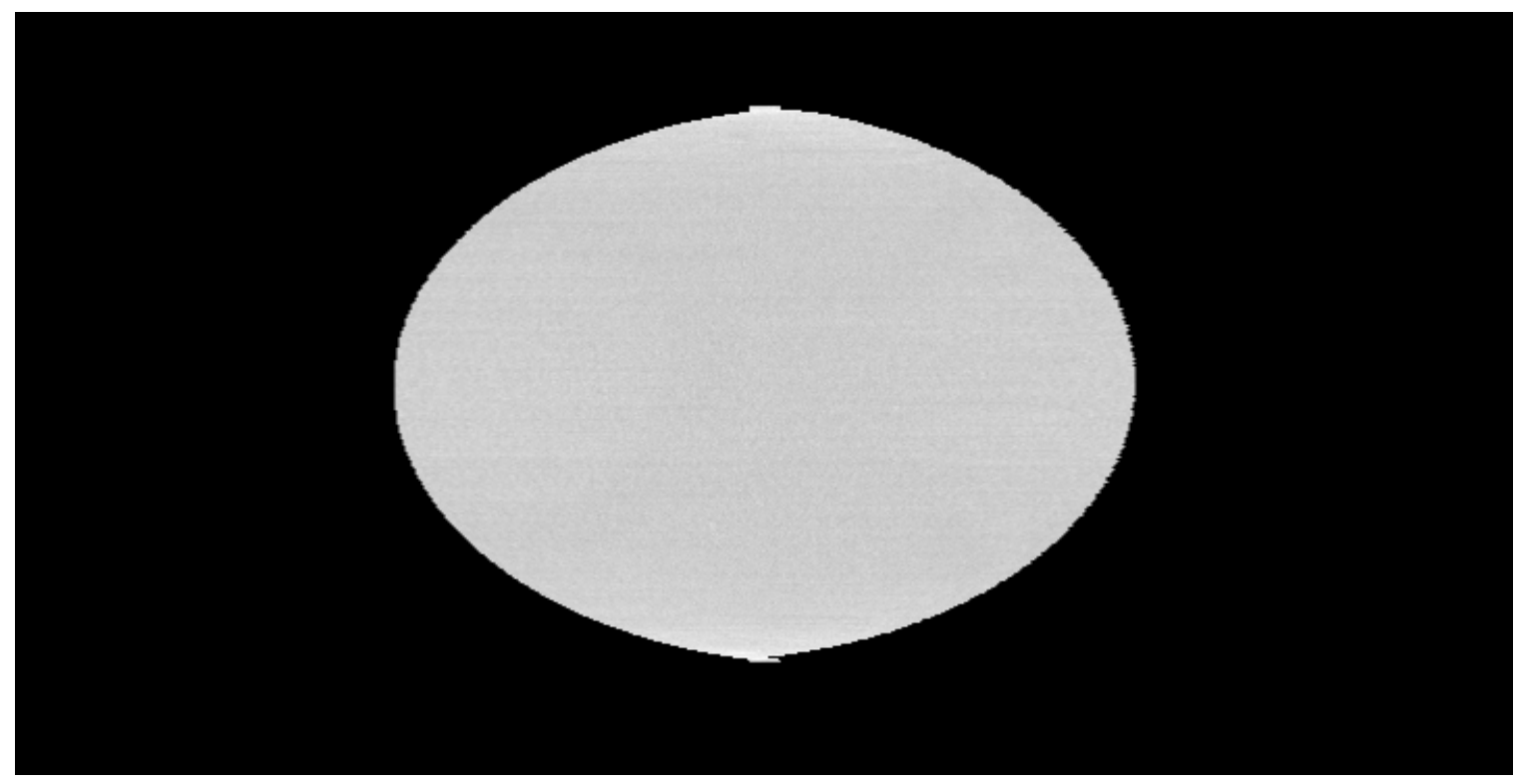

Shell extraction, taken 20 pixels inside of outer surface.

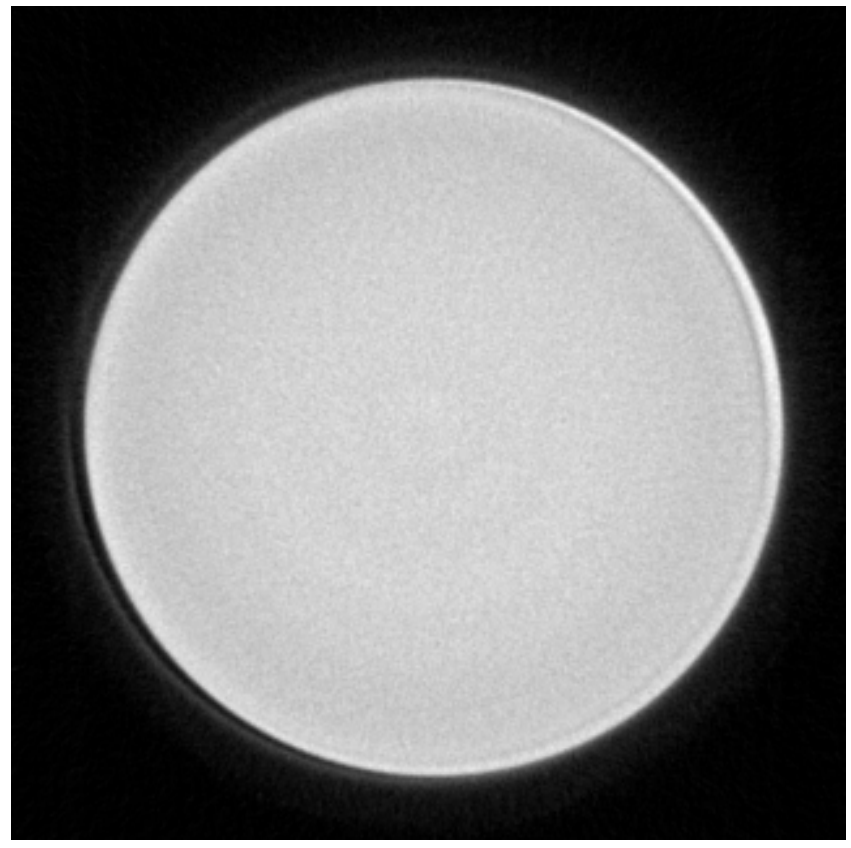

Z slice average of slices $223-227$

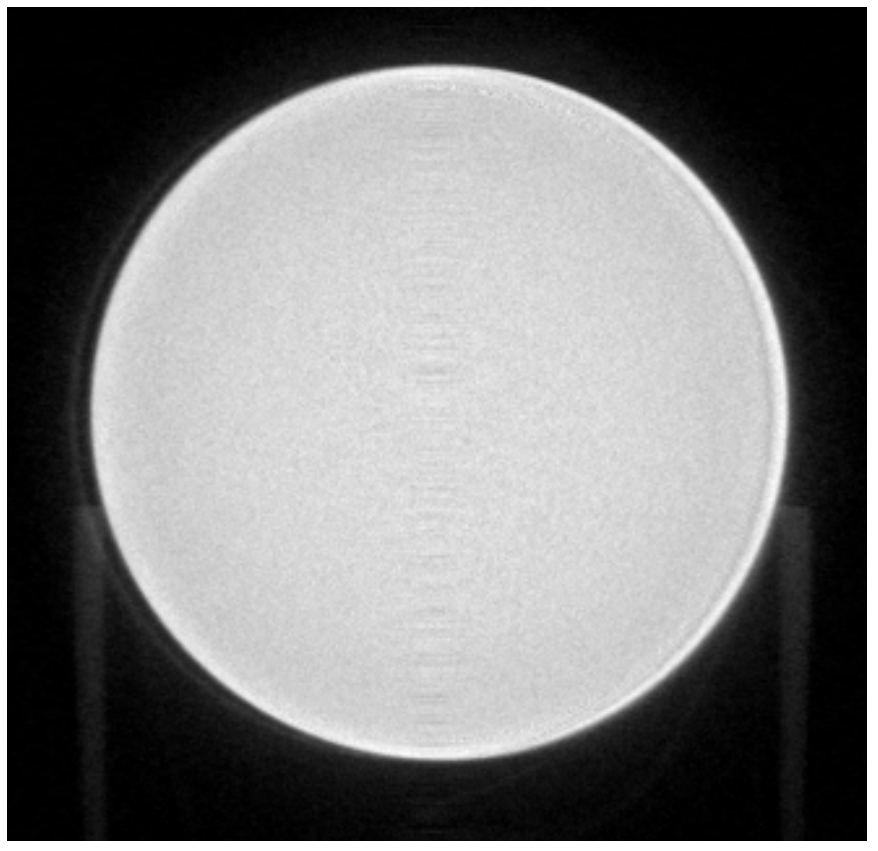

Y slice average of slices 223-227. 
SN 6-92-011

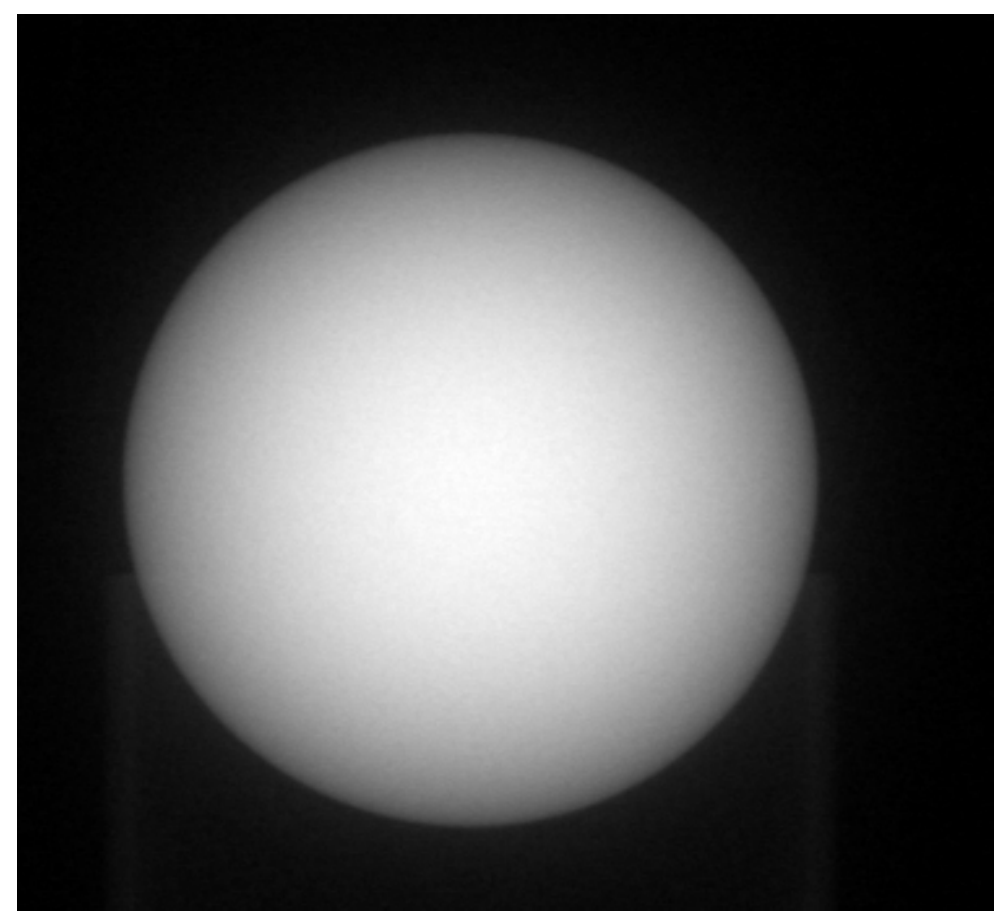

Attenuation radiograph, 0 degree.

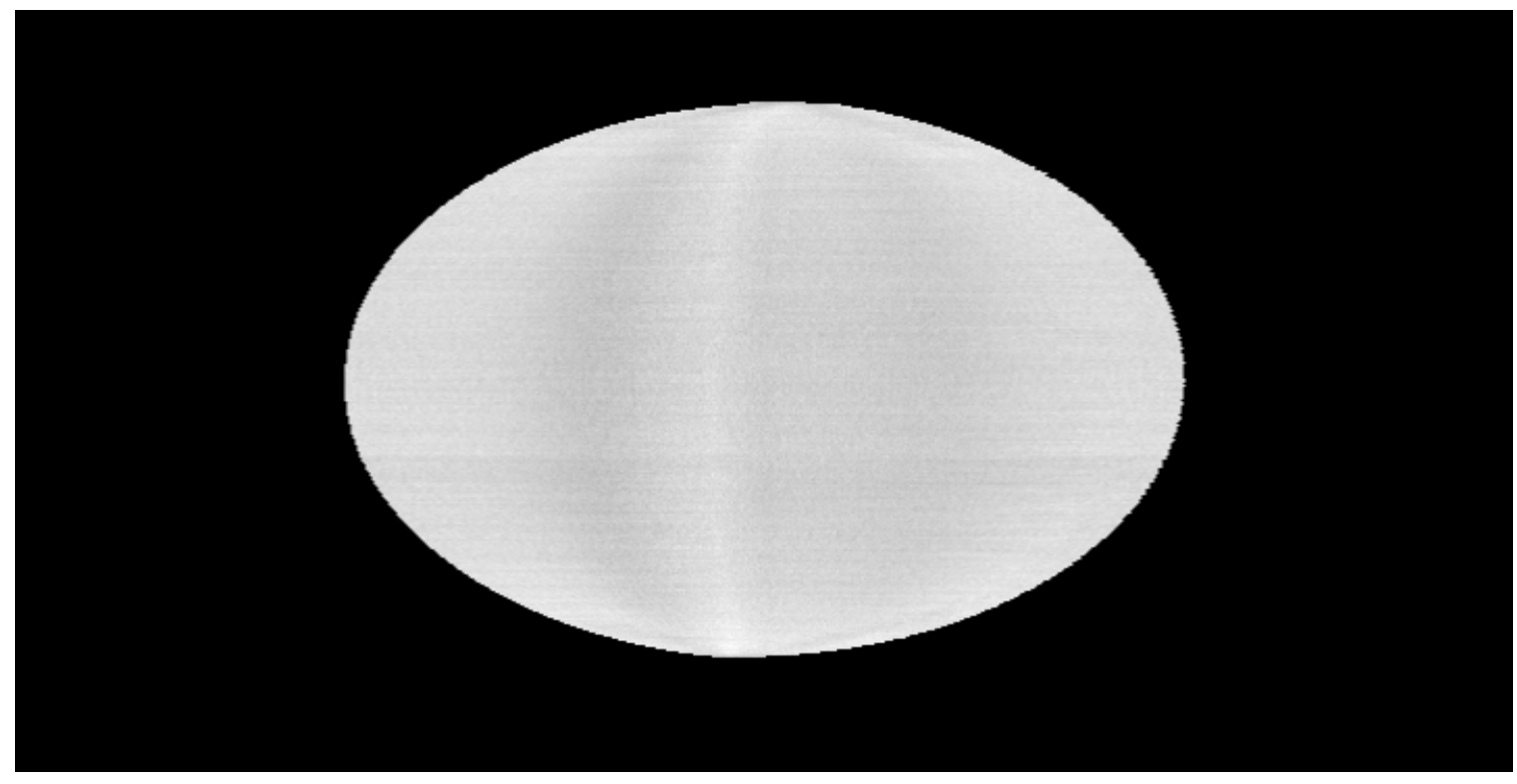

Shell extraction, 5 pixels inside of outer surface. 
SN 6-92-011

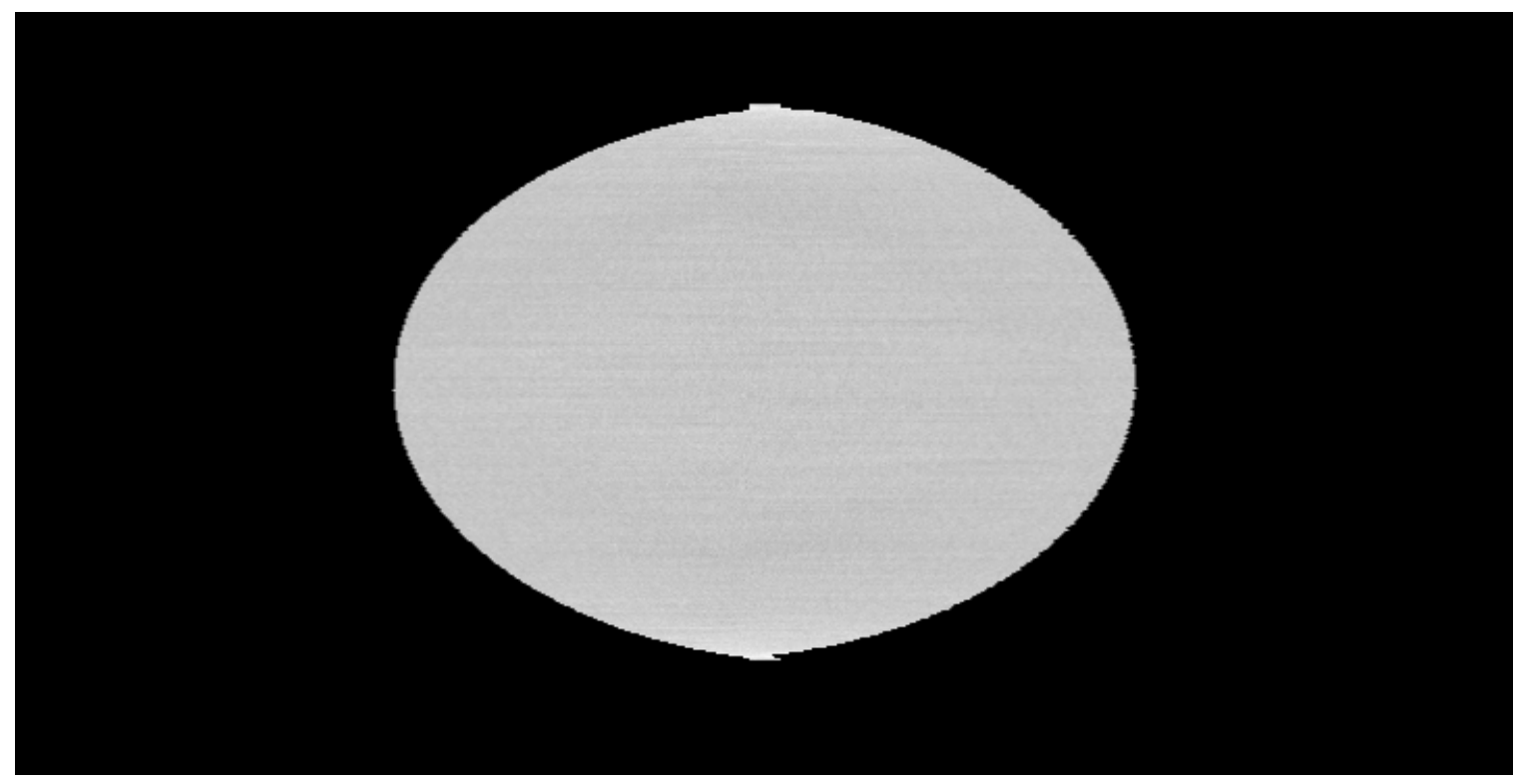

Shell extraction, taken 20 pixels inside of outer surface.

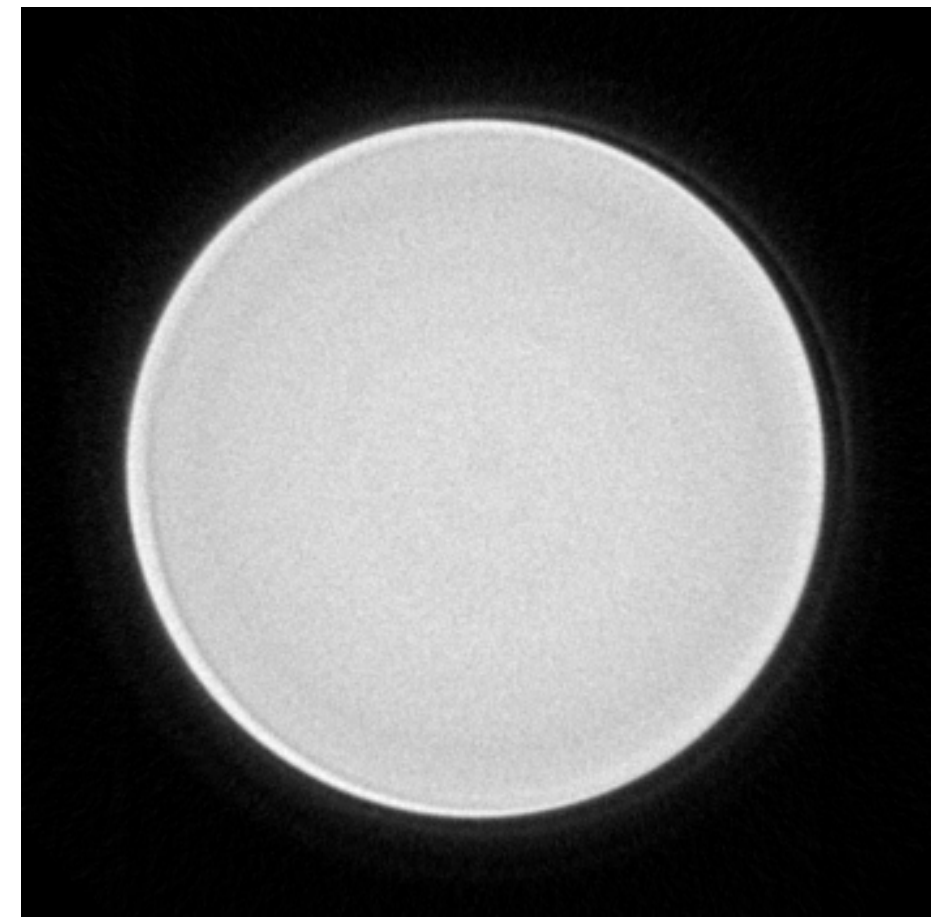

Z slice average of slices 223-227

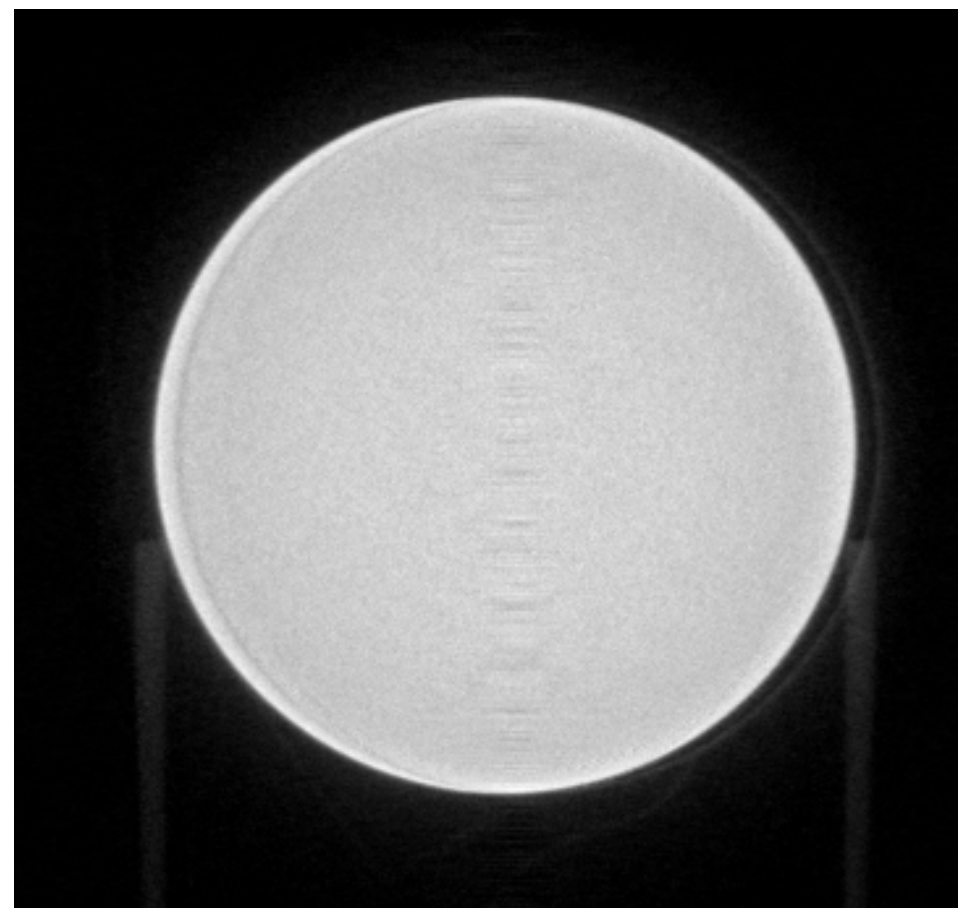

Y slice average of slices 223-227. 
SN 6-92-012

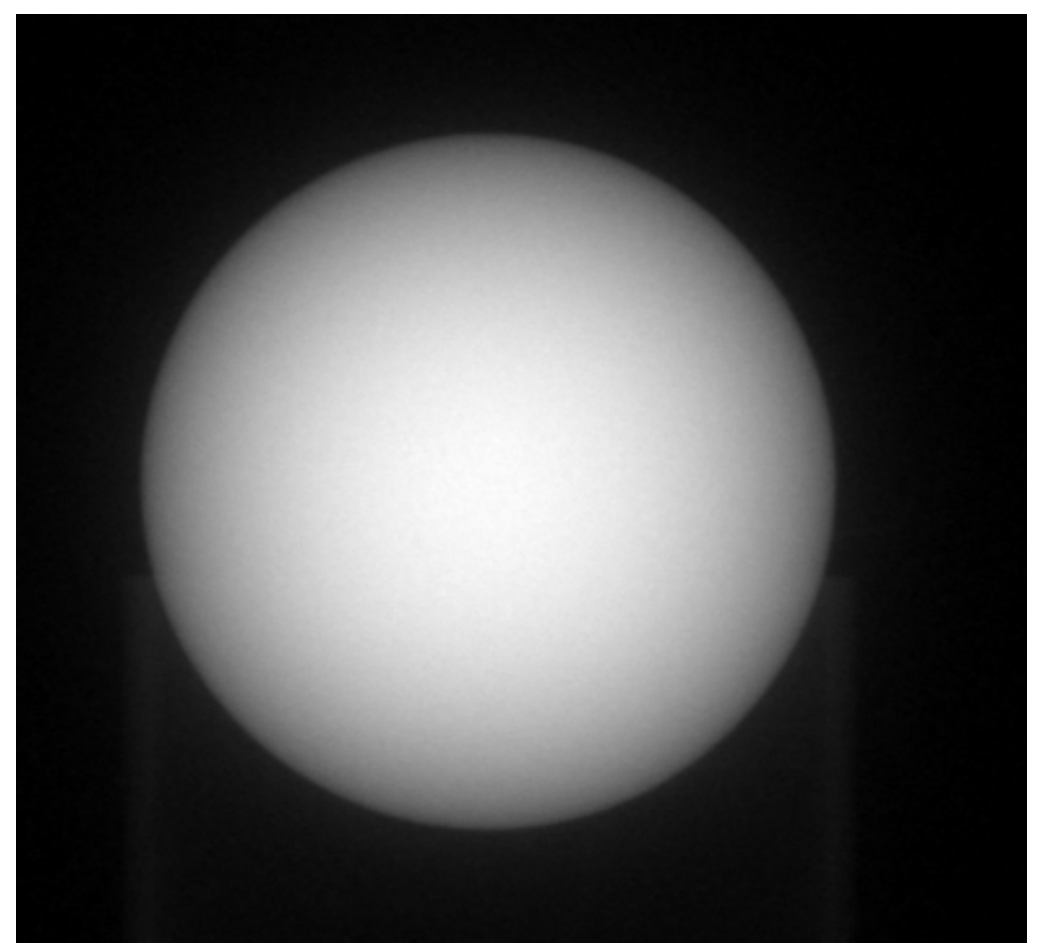

Attenuation radiograph, 0 degree.

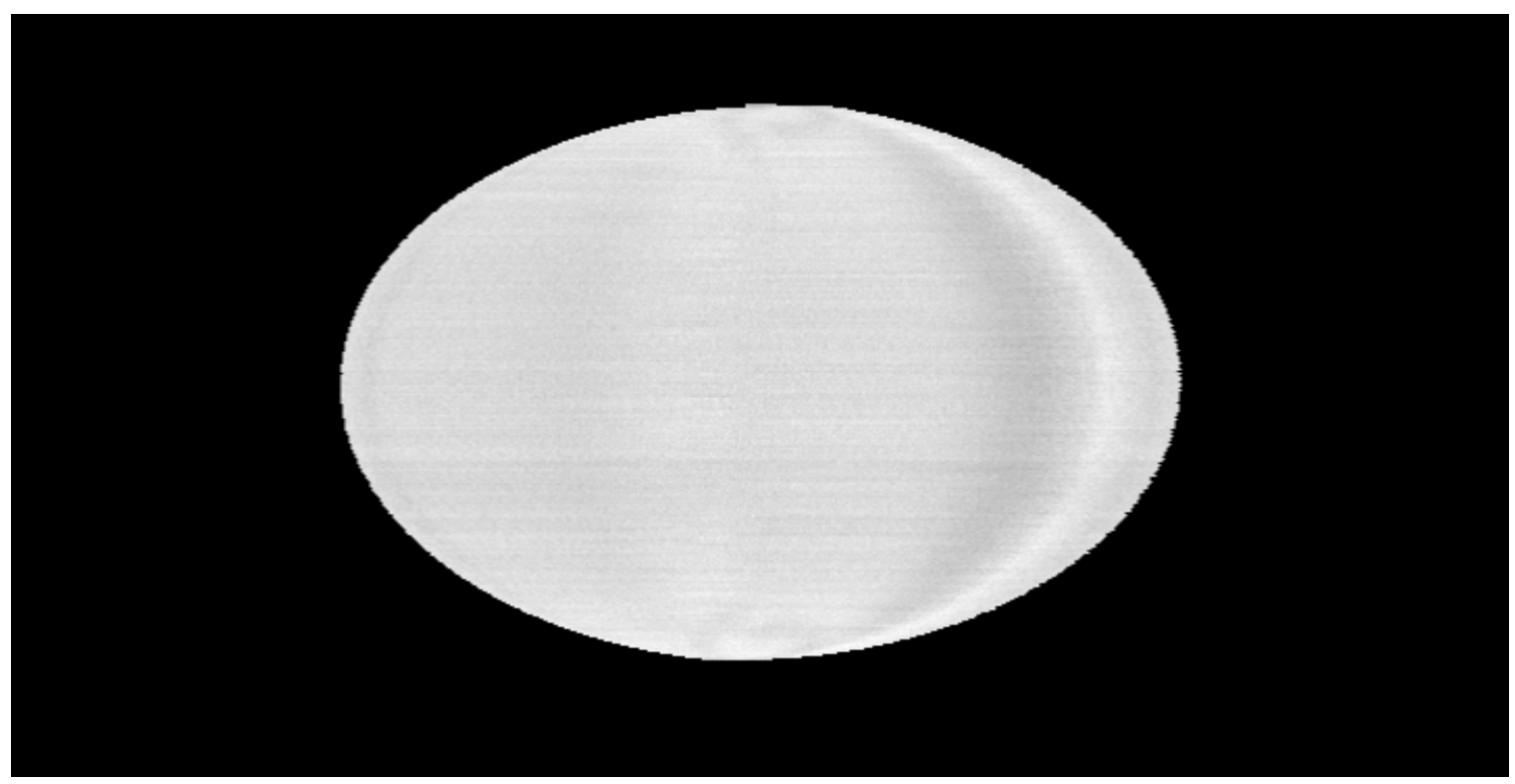

Shell extraction, 5 pixels inside of outer surface. 
SN 6-92-012

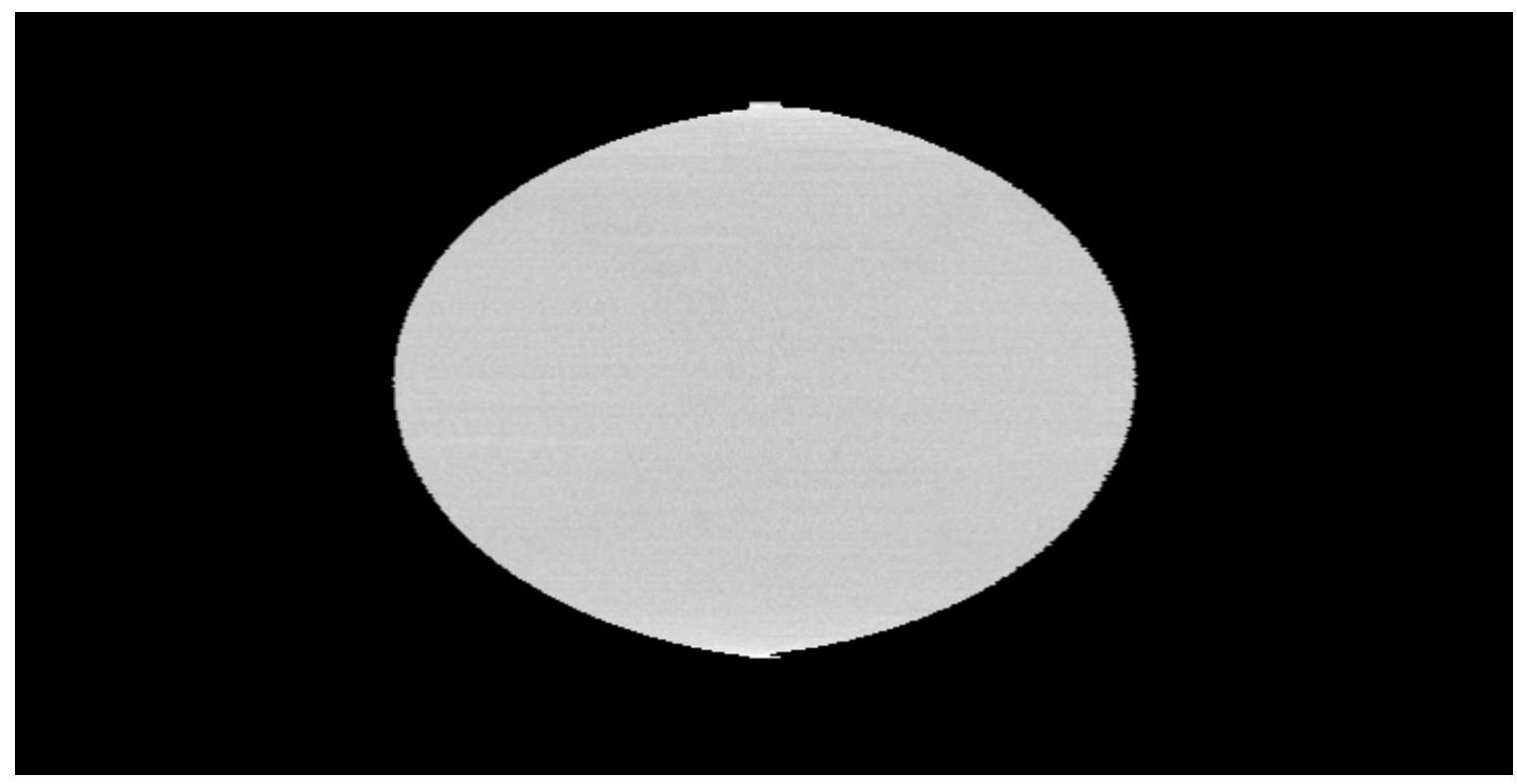

Shell extraction, taken 20 pixels inside of outer surface.

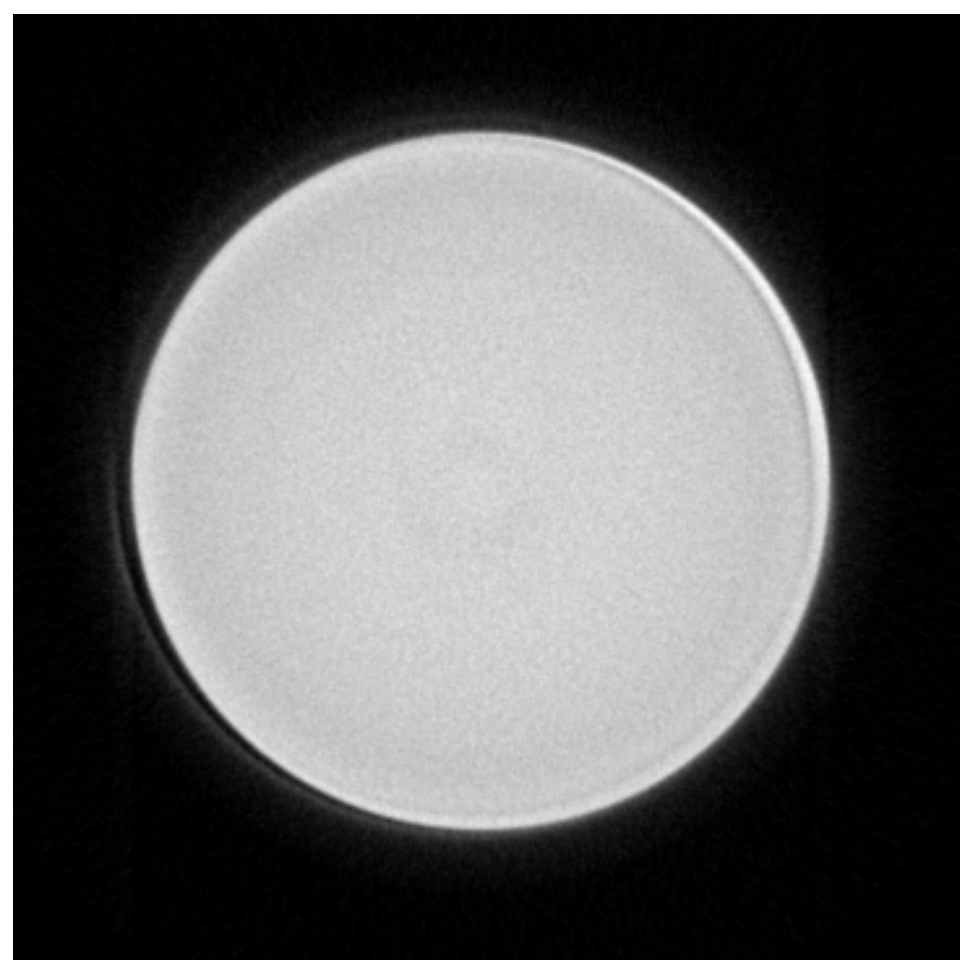

Z slice average of slices 223-227

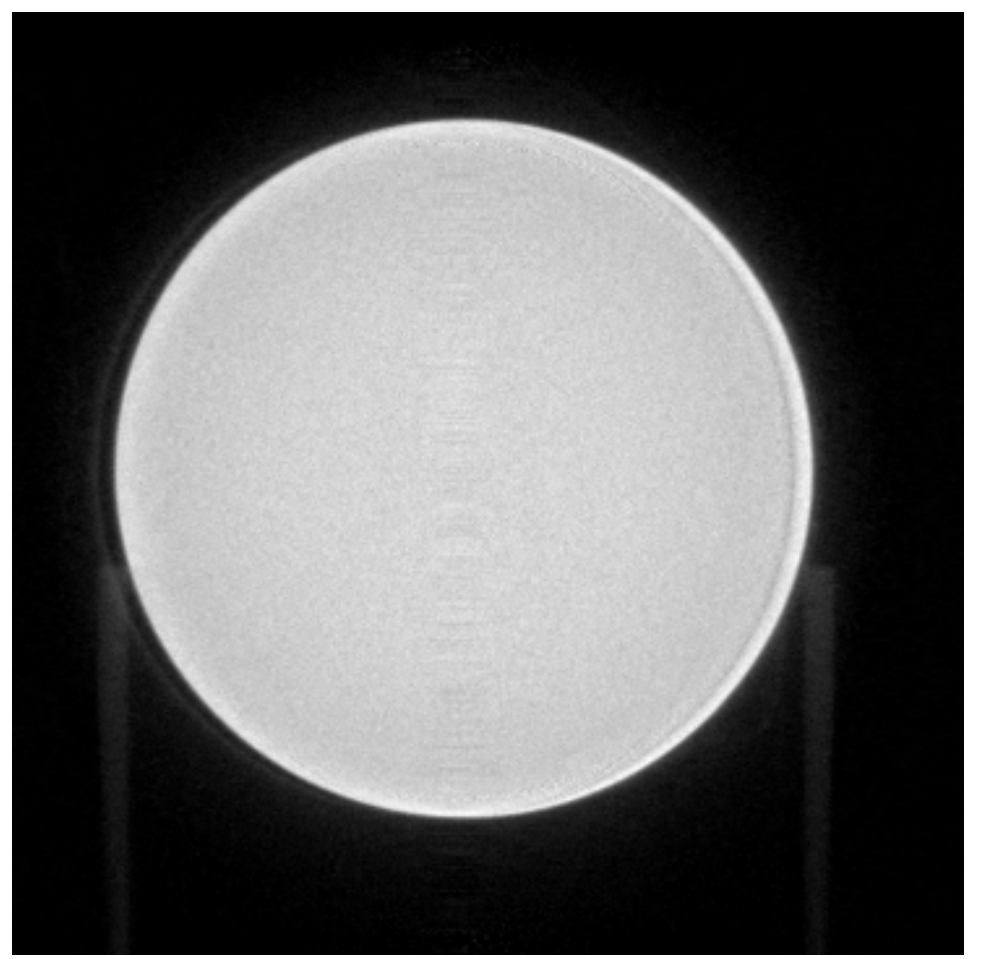

Y slice average of slices 223-227. 
SN 6-92-013

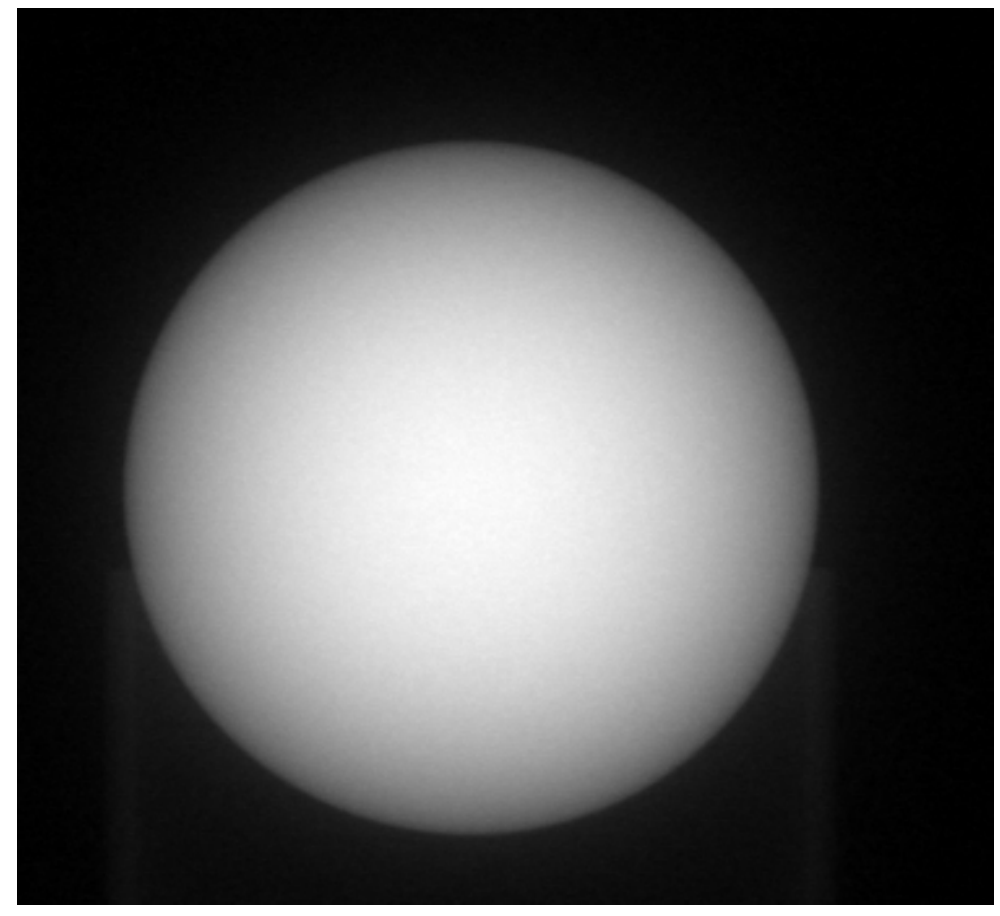

Attenuation radiograph, 0 degree.

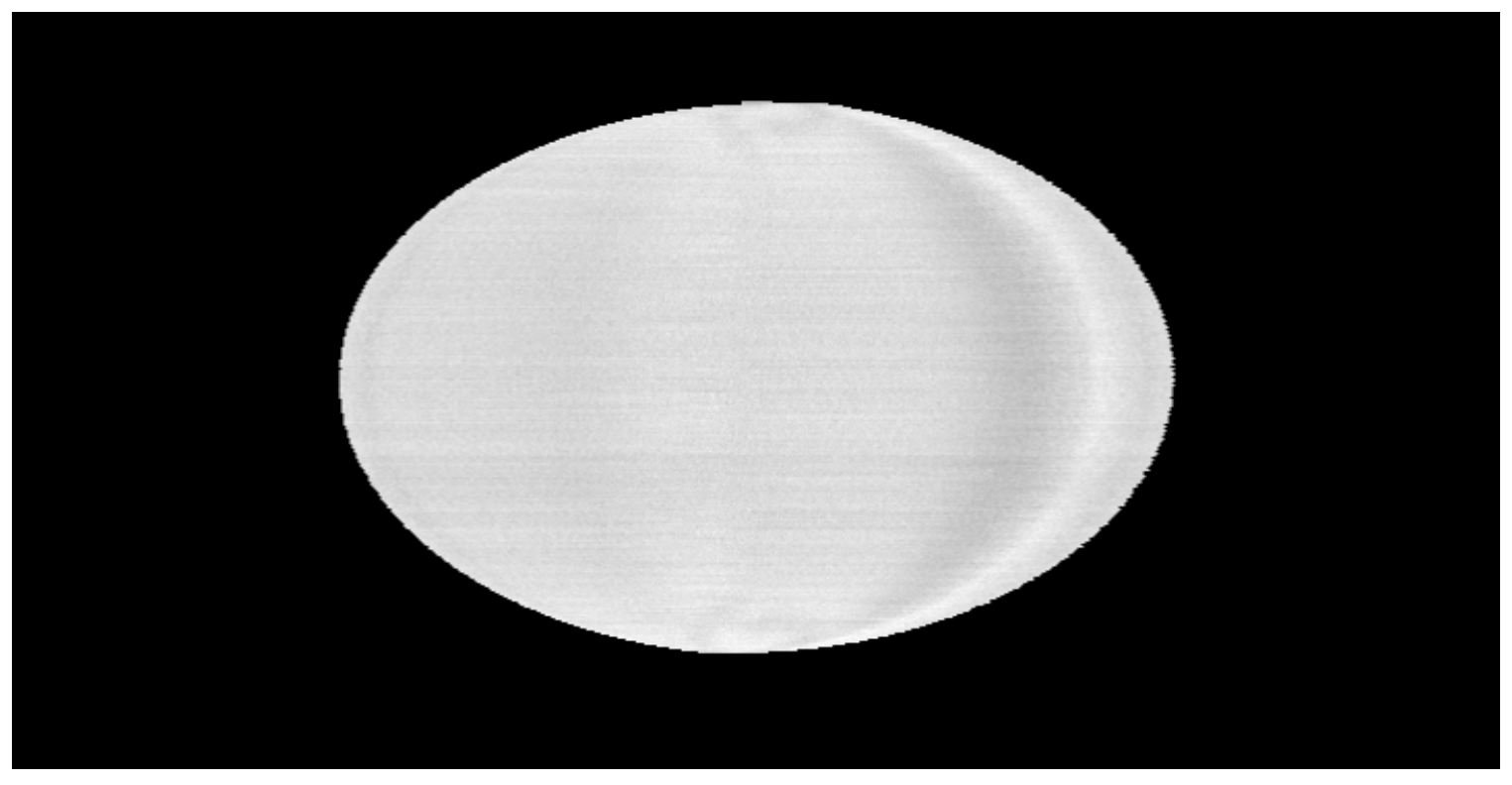

Shell extraction, 5 pixels inside of outer surface 
SN 6-92-013

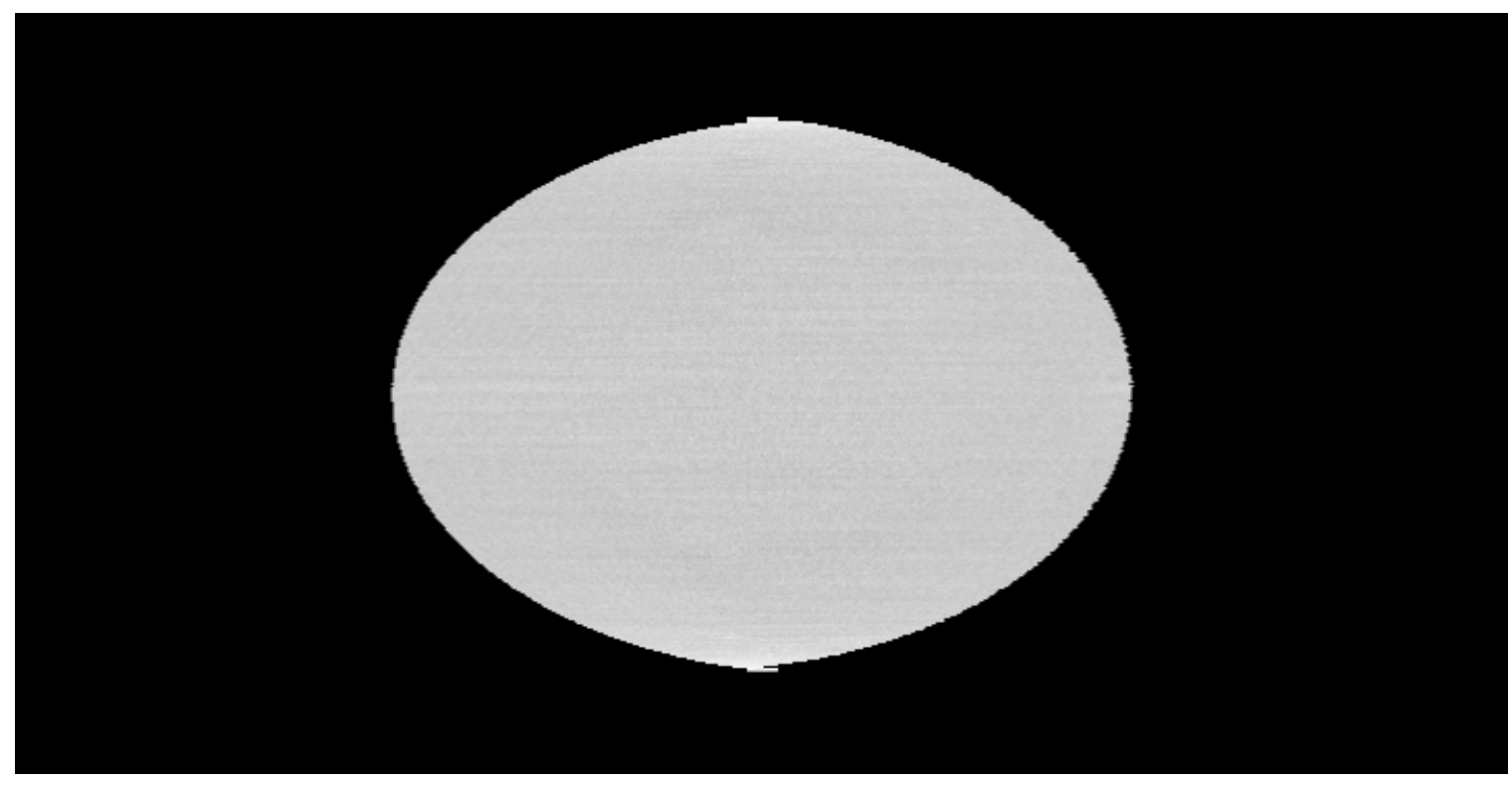

Shell extraction, taken 20 pixels inside of outer surface.

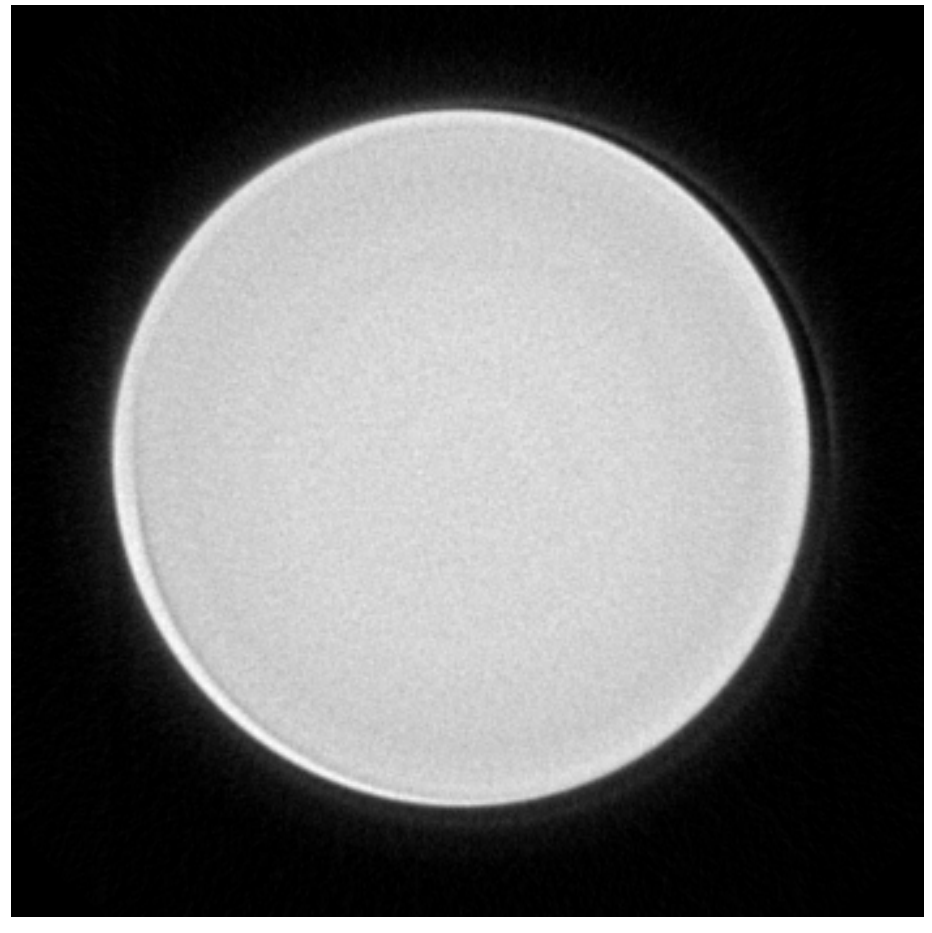

Z slice average of slices 223-227

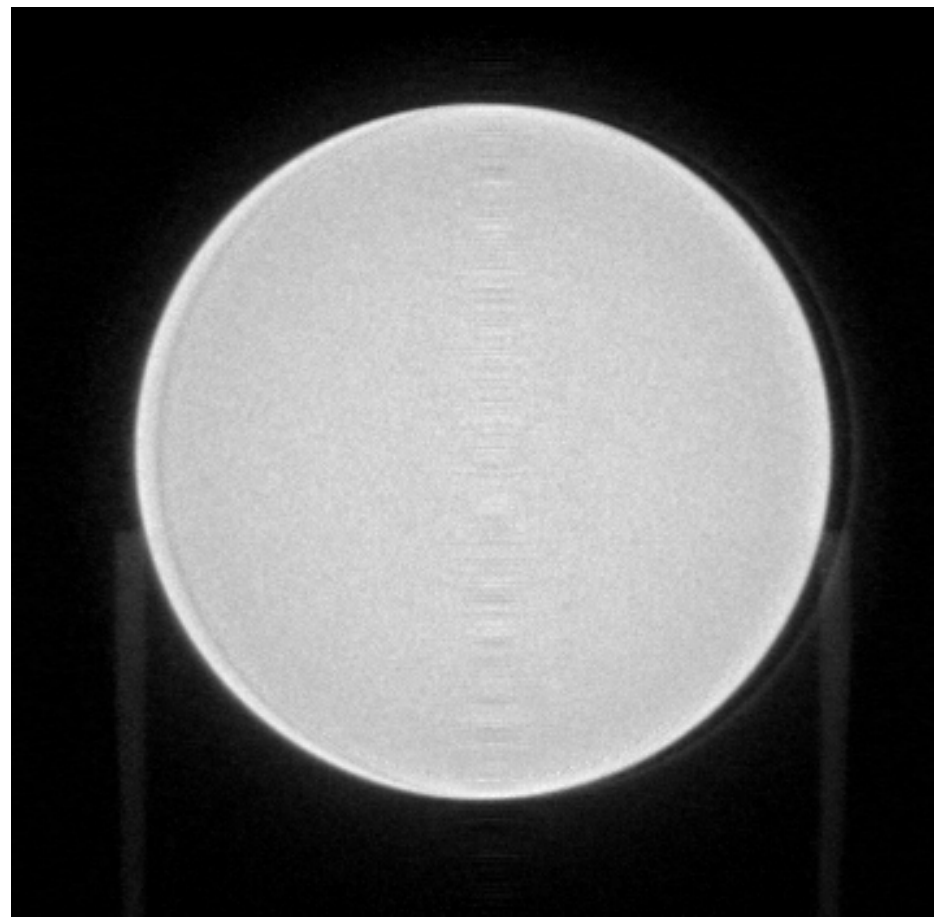

Y slice average of slices 223-227. 
SN 6-92-014

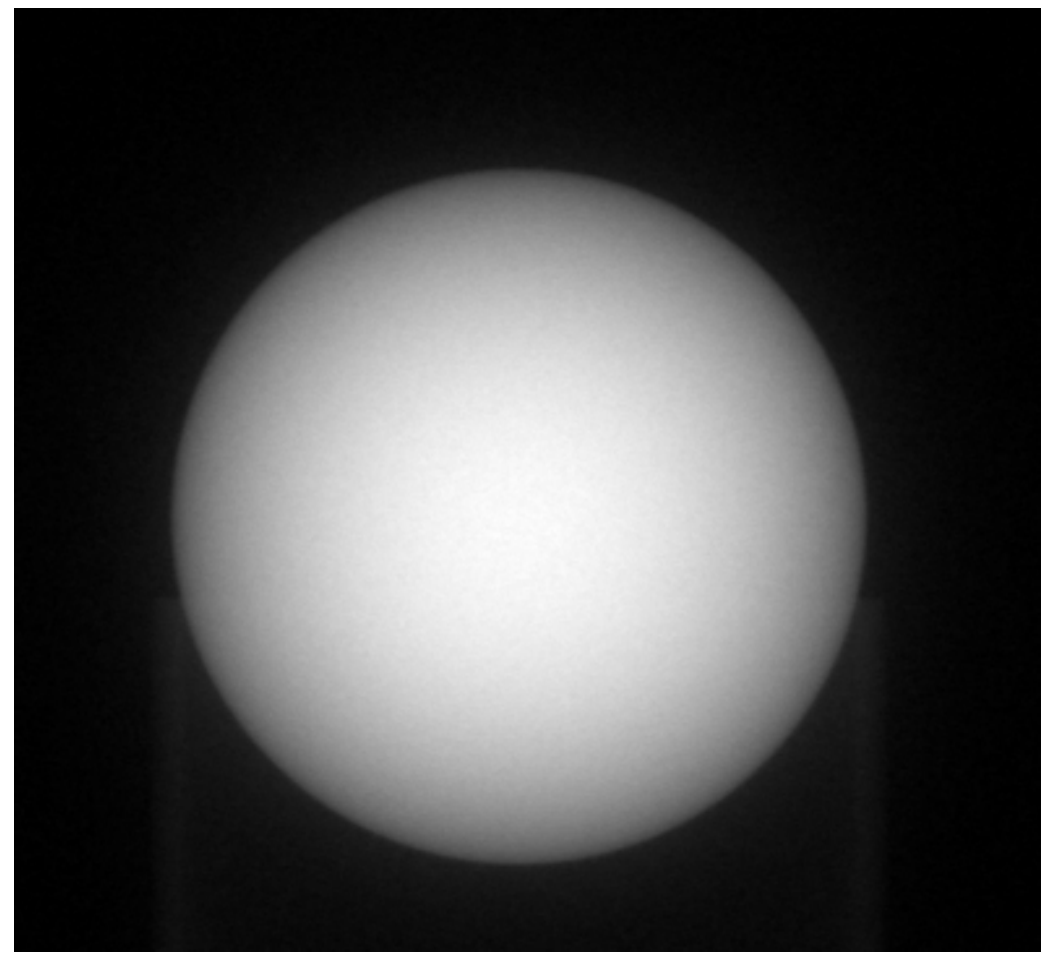

Attenuation radiograph, 0 degree.

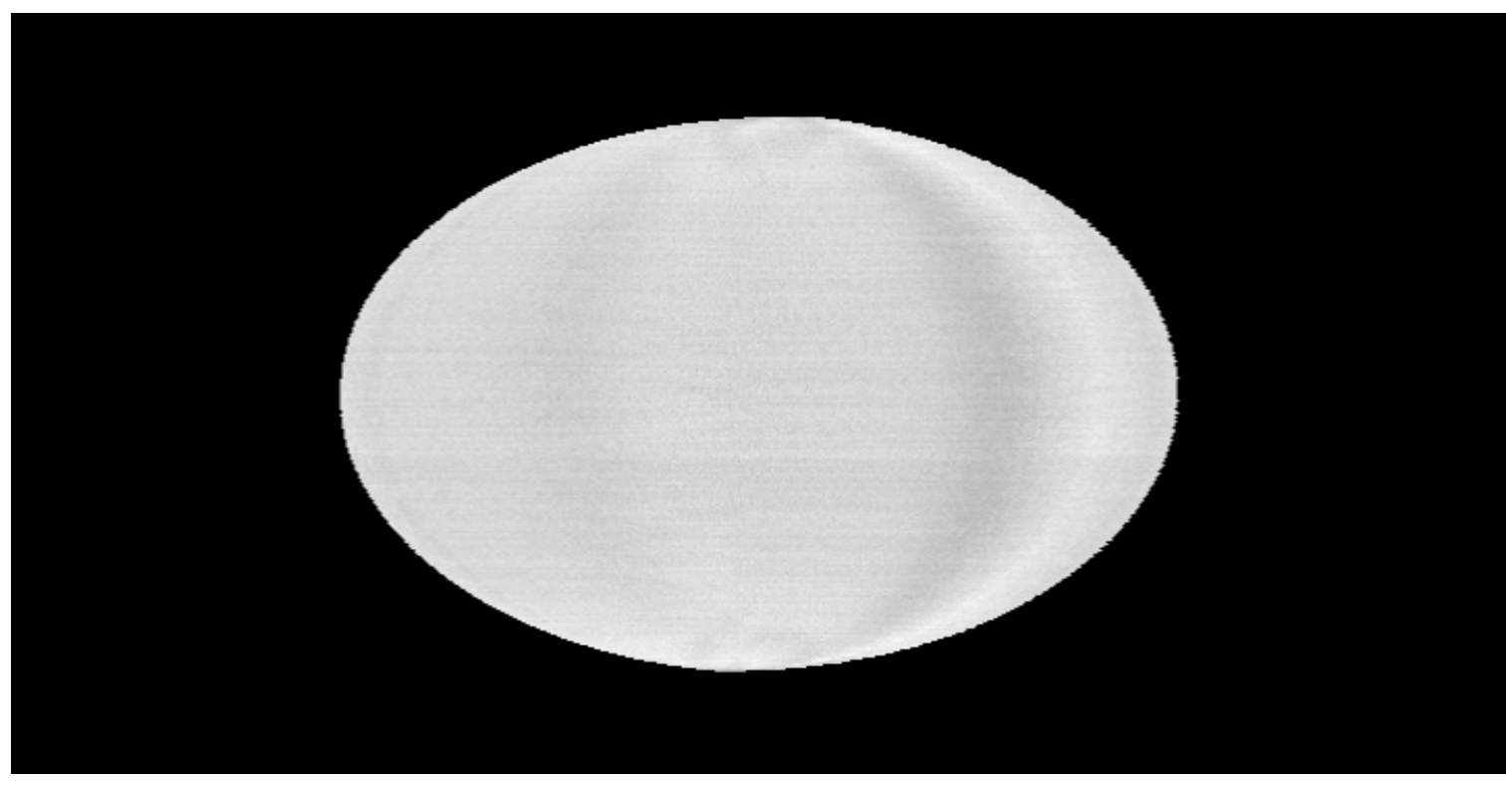

Shell extraction, 5 pixels inside of outer surface. 
SN 6-92-014

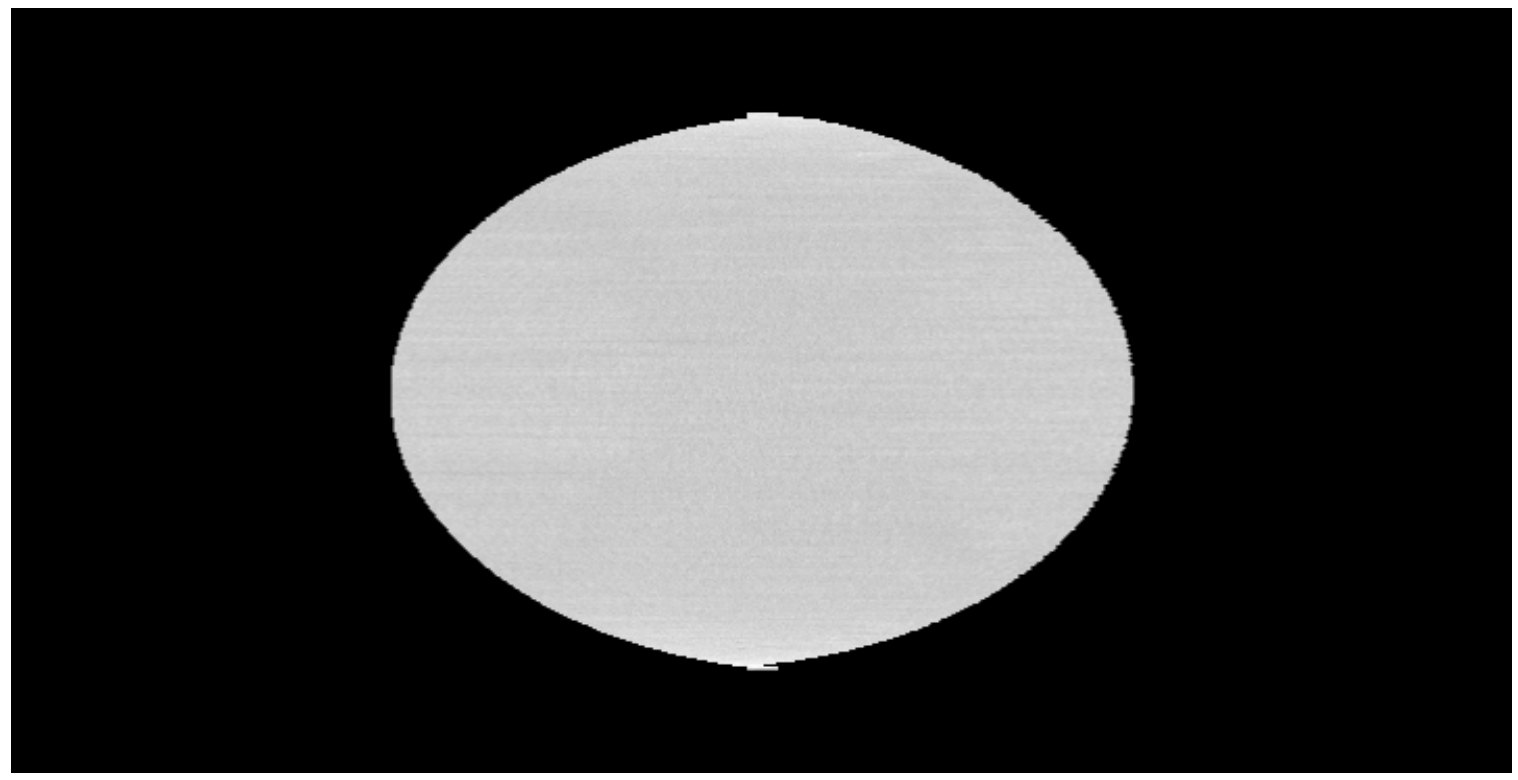

Shell extraction, taken 20 pixels inside of outer surface.

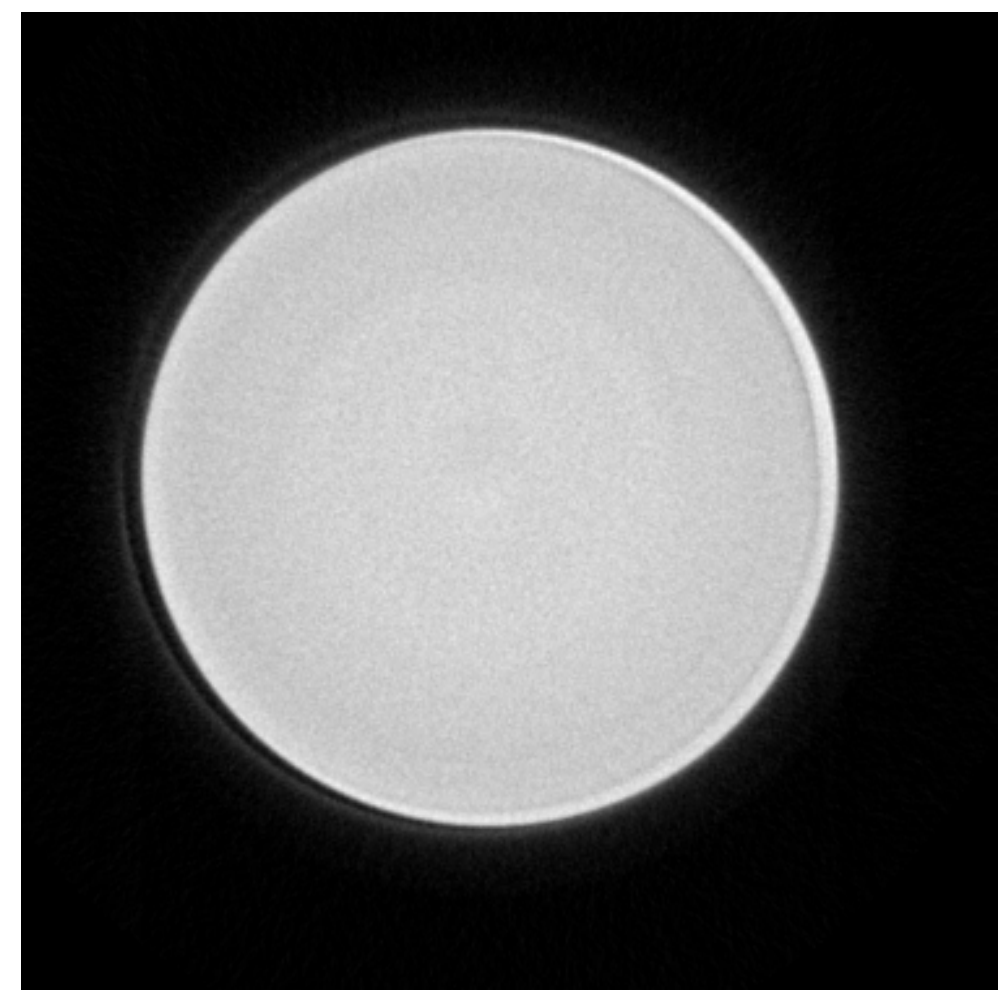

Z slice average of slices $223-227$

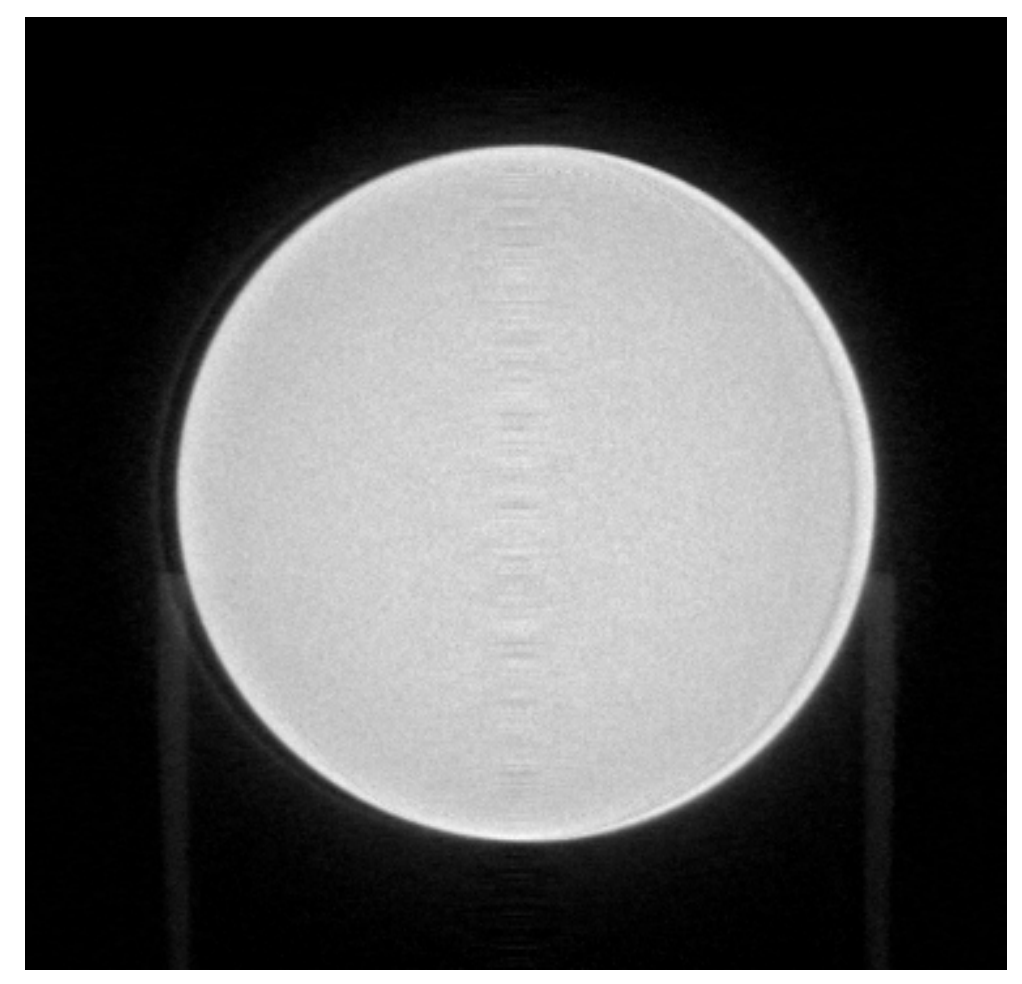

Y slice average of slices 223-227. 
SN 6-92-015

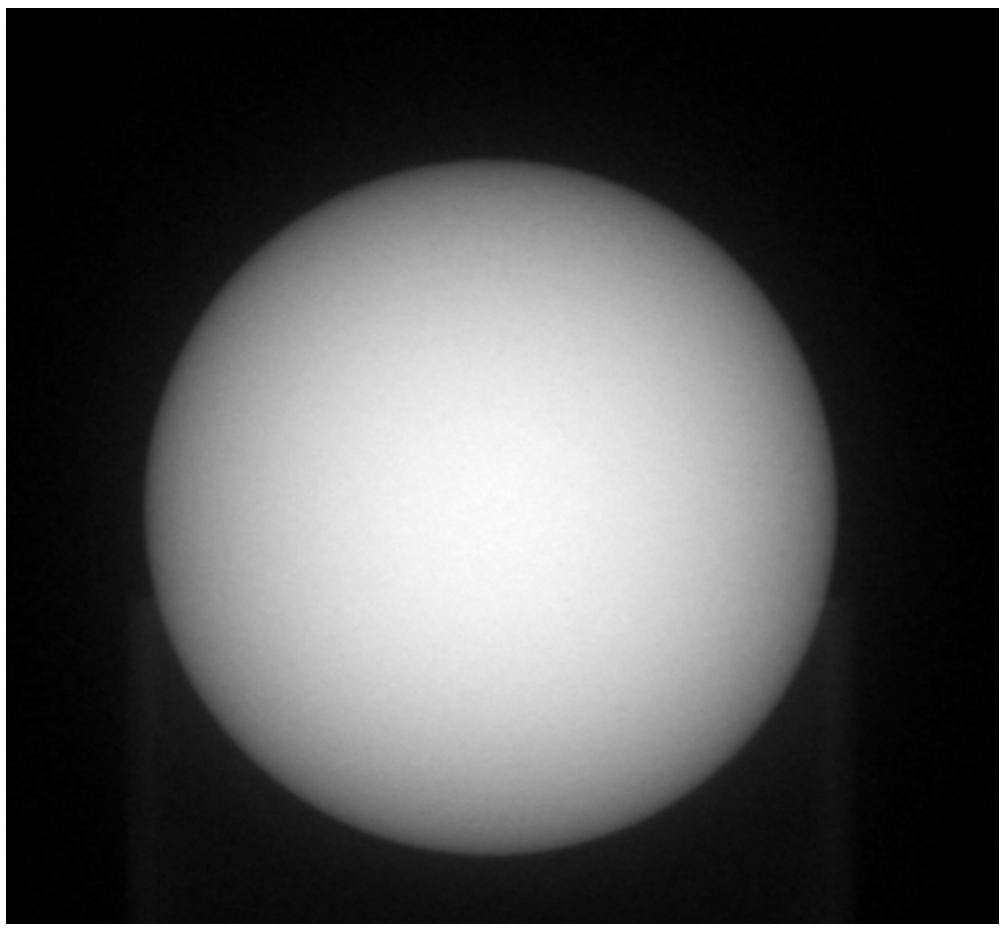

Attenuation radiograph, 0 degree.

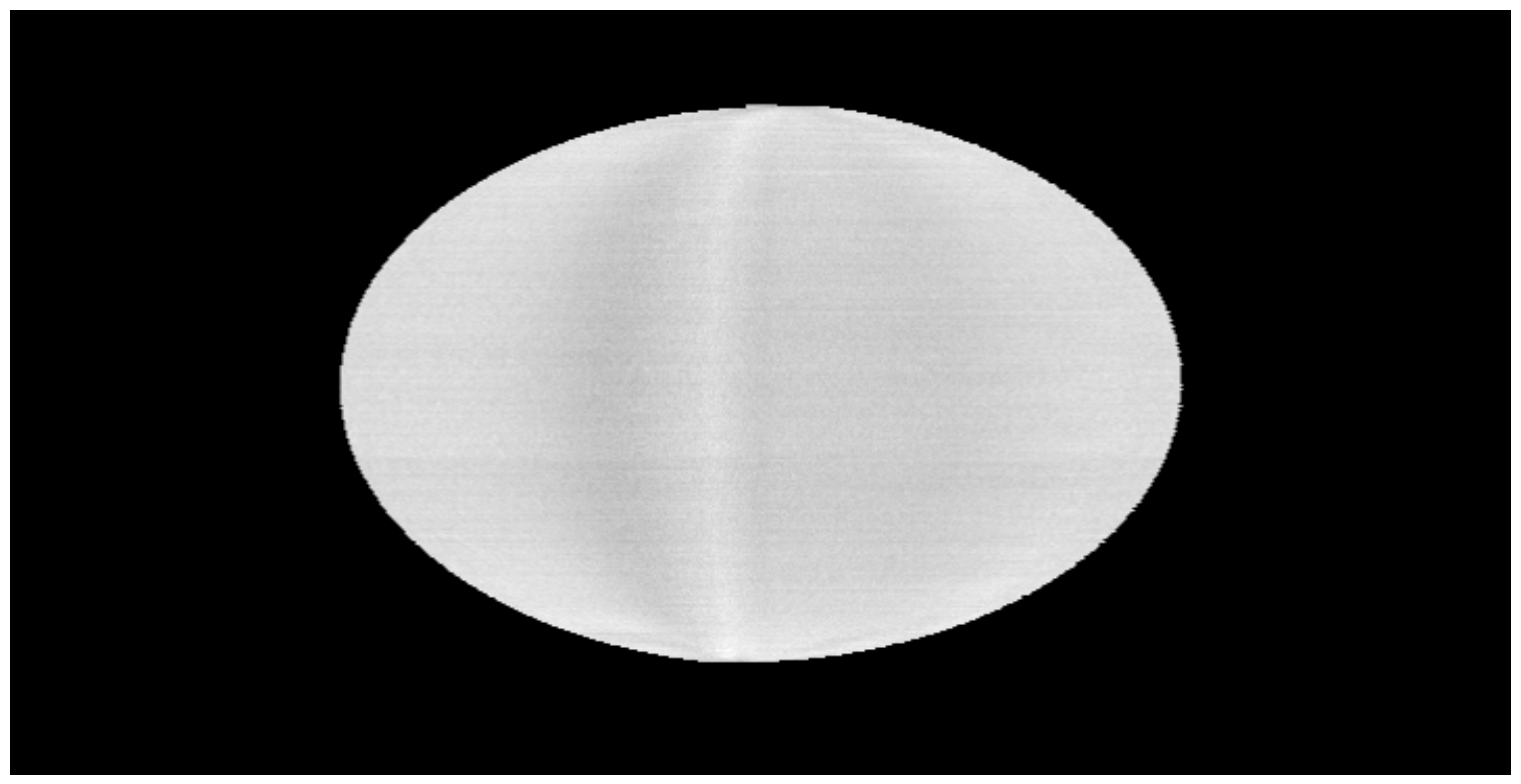

Shell extraction, 5 pixels inside of outer surface. 
SN 6-92-015

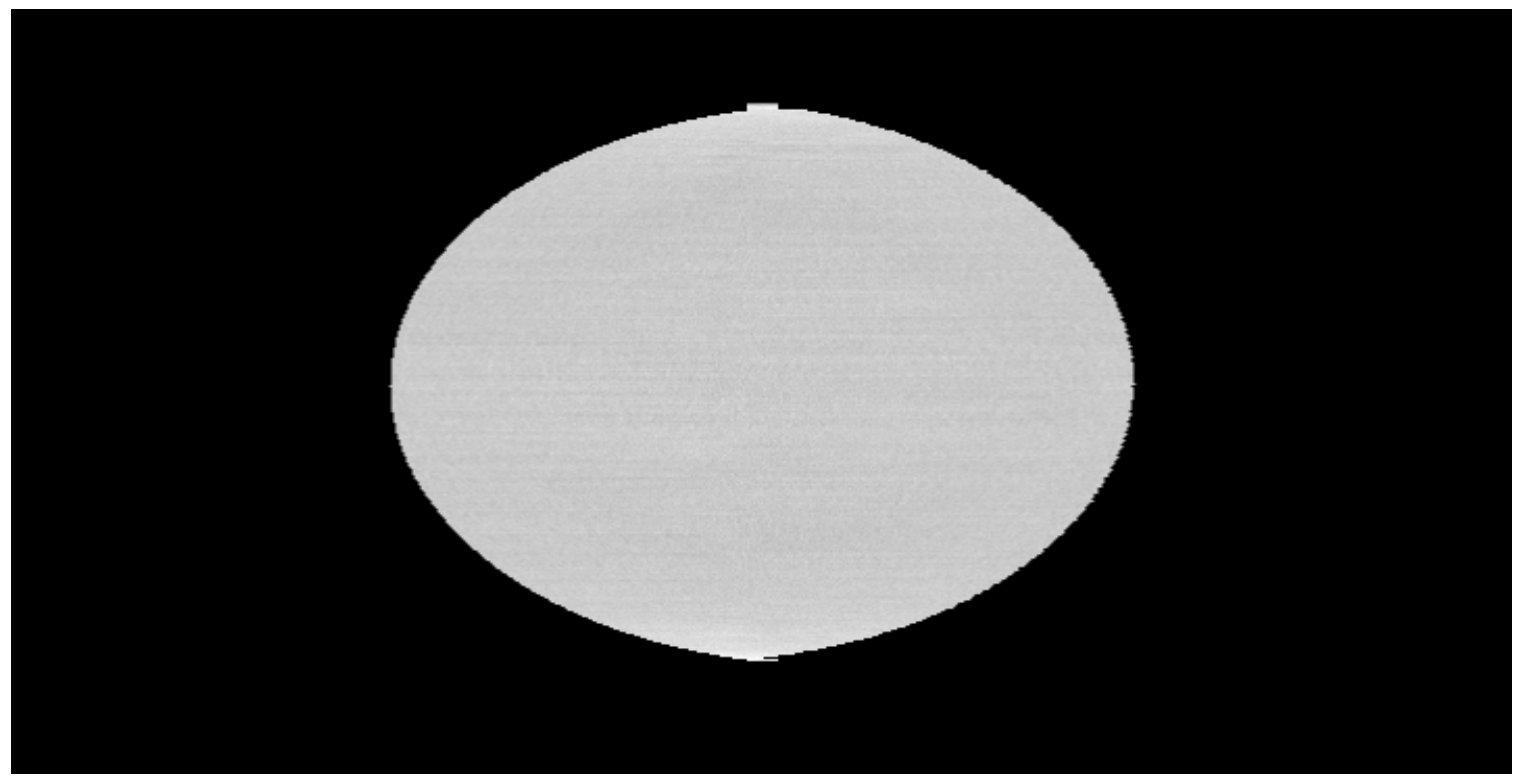

Shell extraction, taken 20 pixels inside of outer surface.

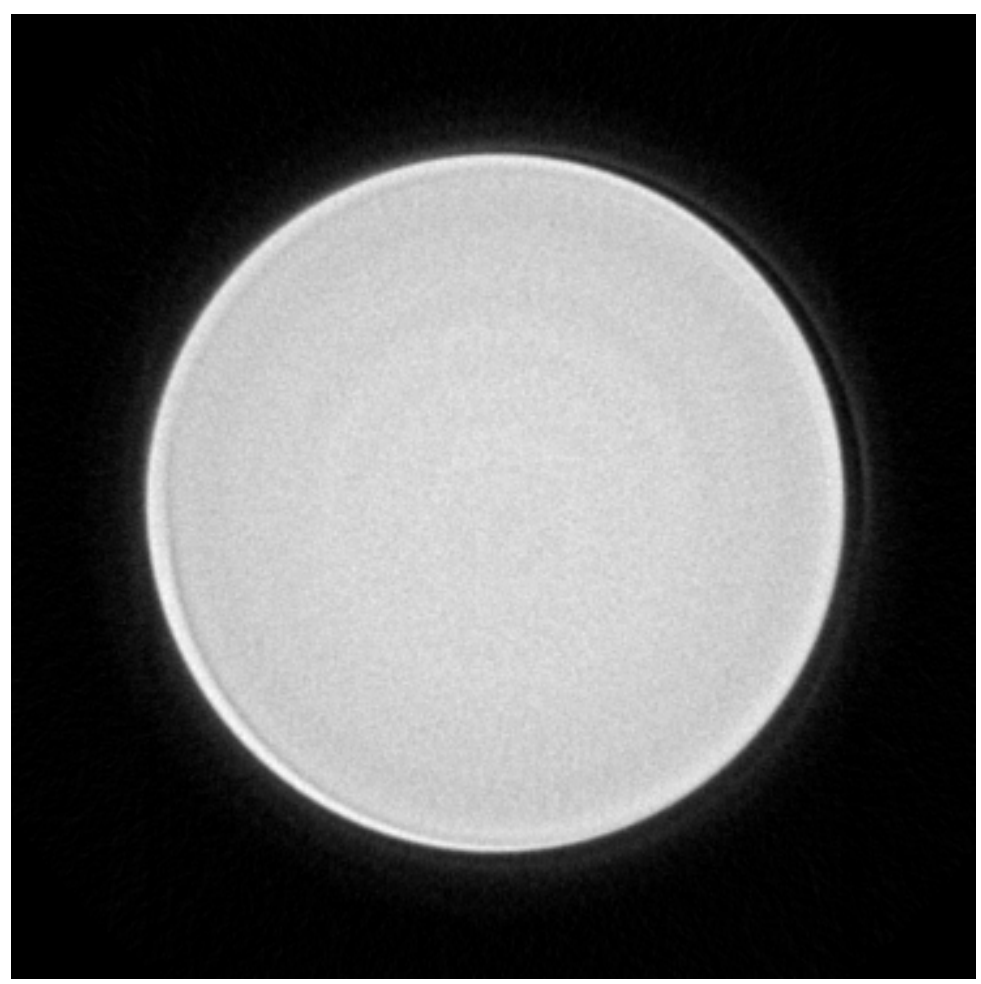

Z slice average of slices 223-227

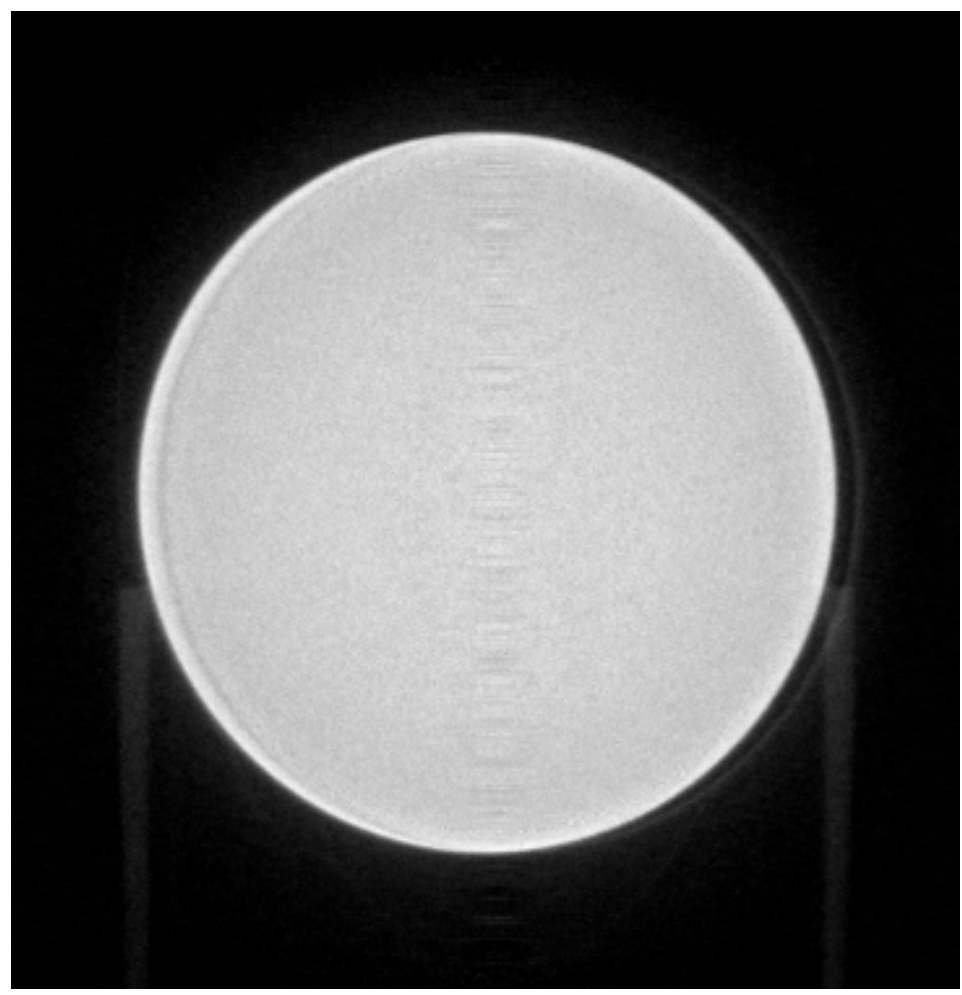

Y slice average of slices 223-227. 
SN 6-92-016

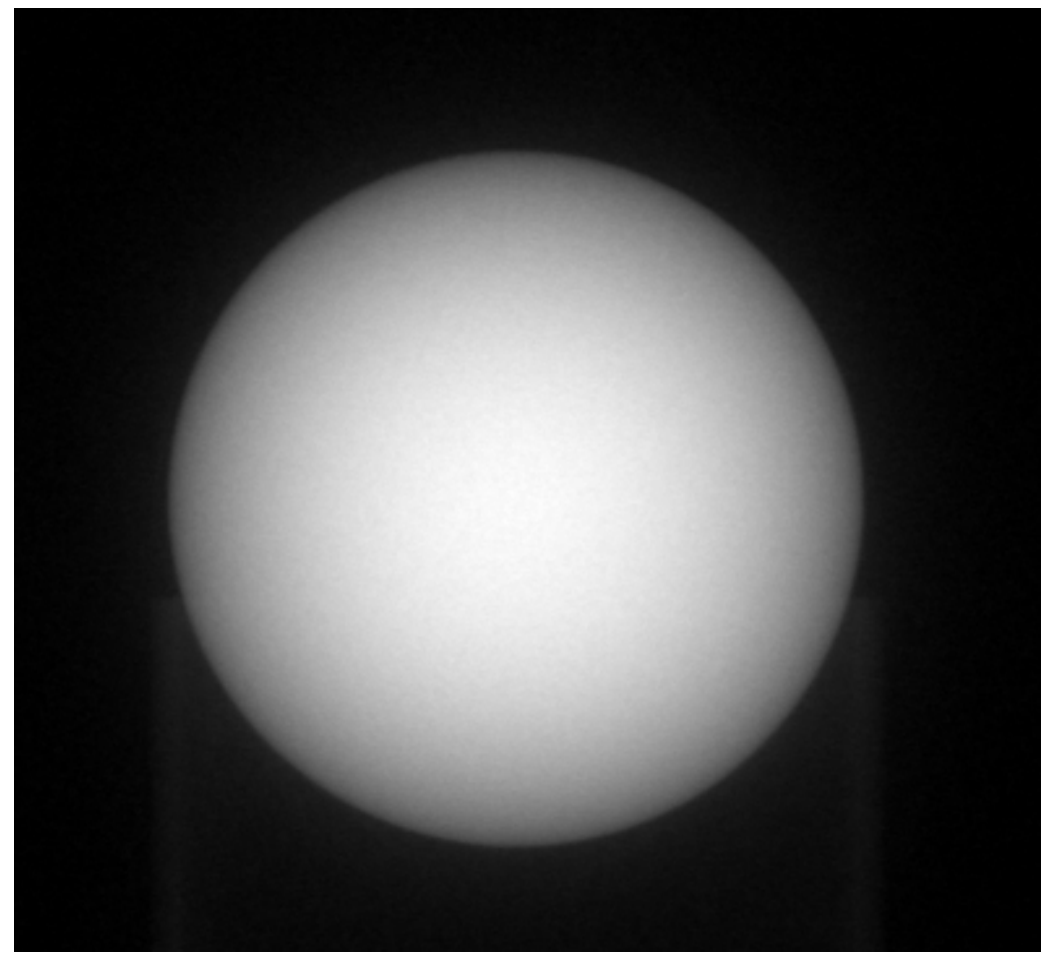

Attenuation radiograph, 0 degree.

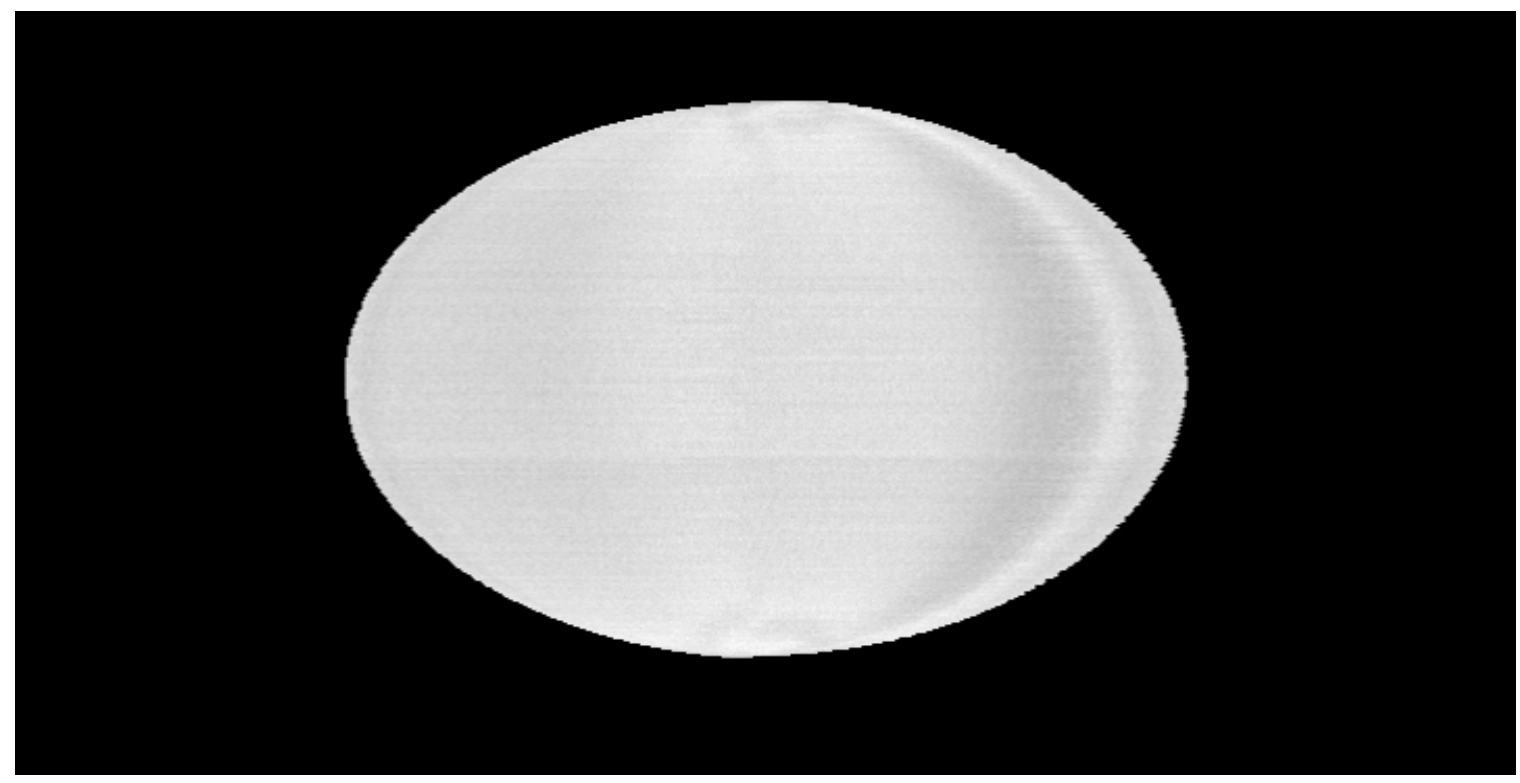

Shell extraction, 5 pixels inside of outer surface. 
SN 6-92-016

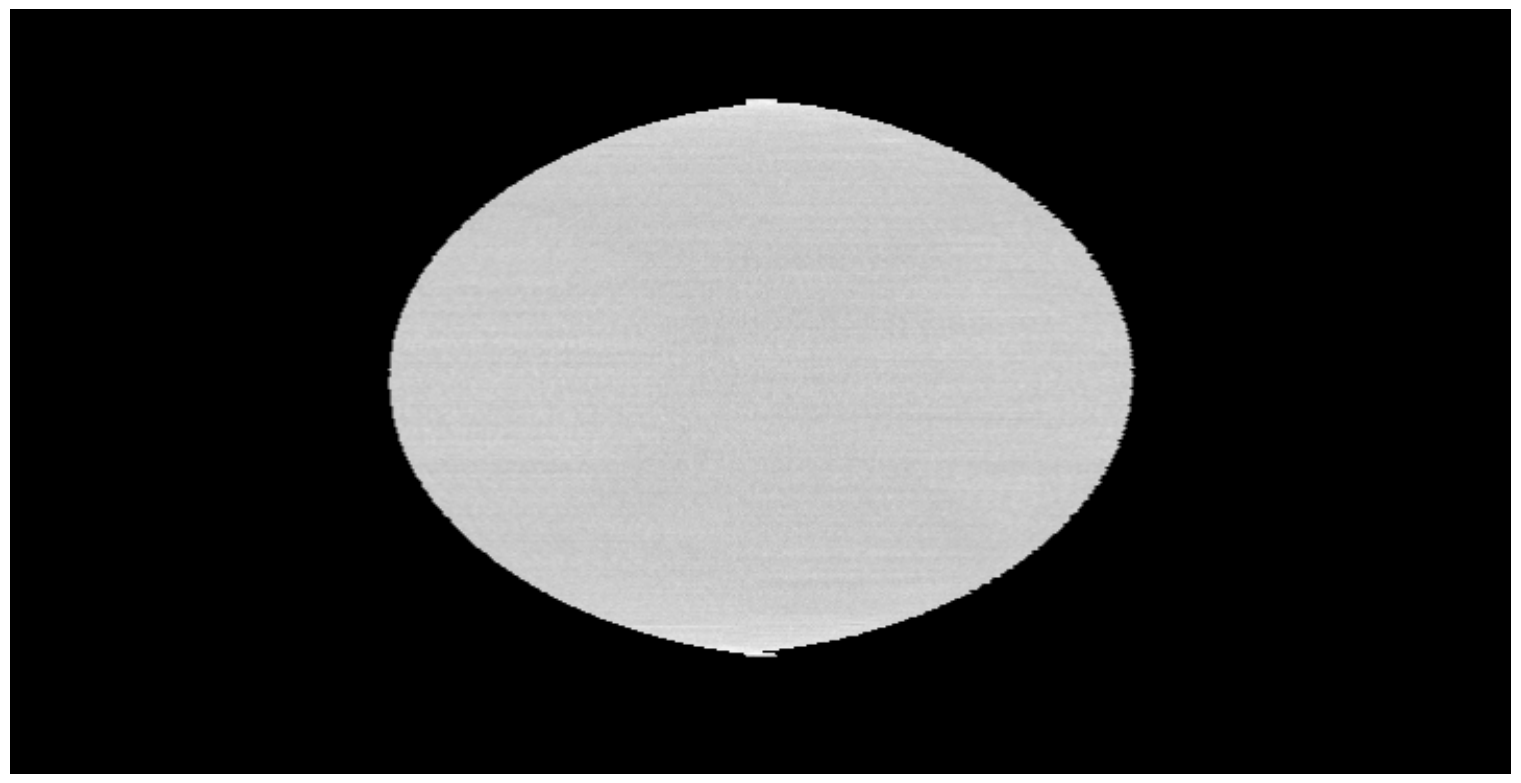

Shell extraction, taken 20 pixels inside of outer surface.
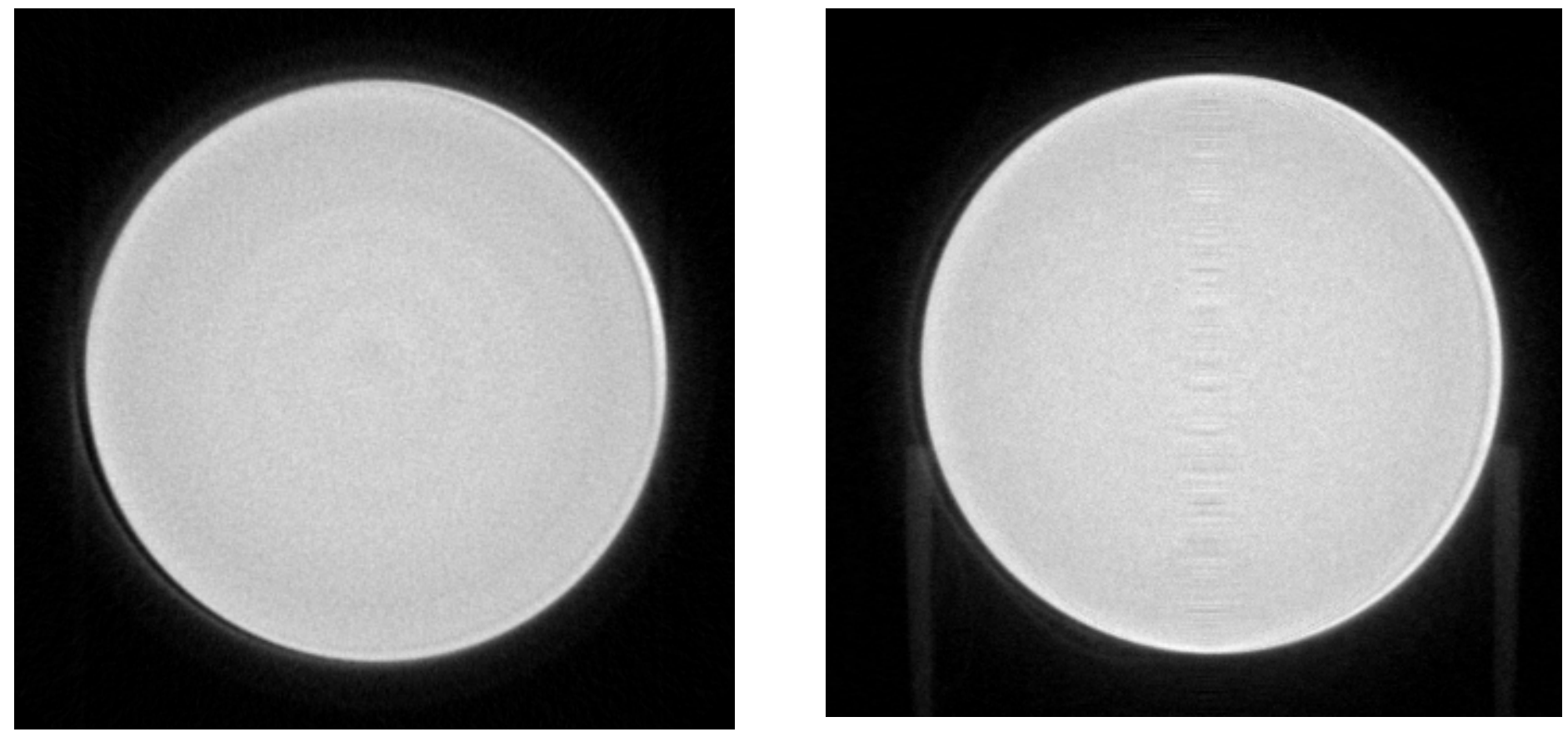

Z slice average of slices $223-227$

Y slice average of slices 223-227. 


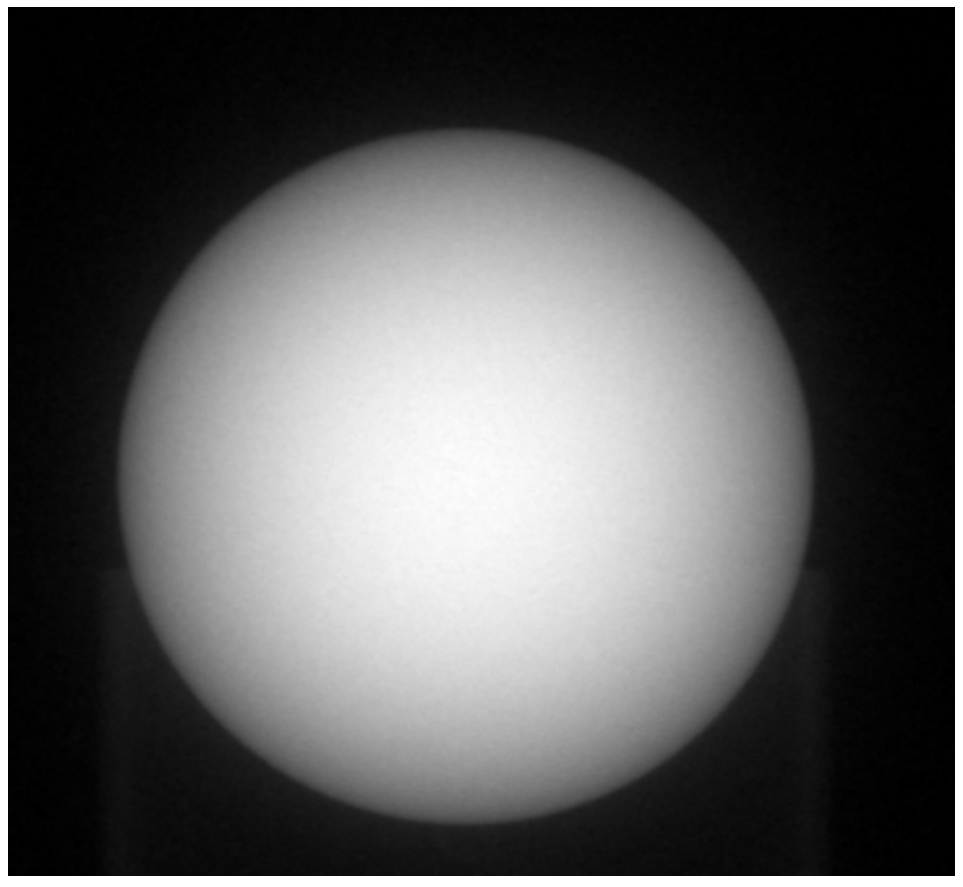

Attenuation radiograph, 0 degree.

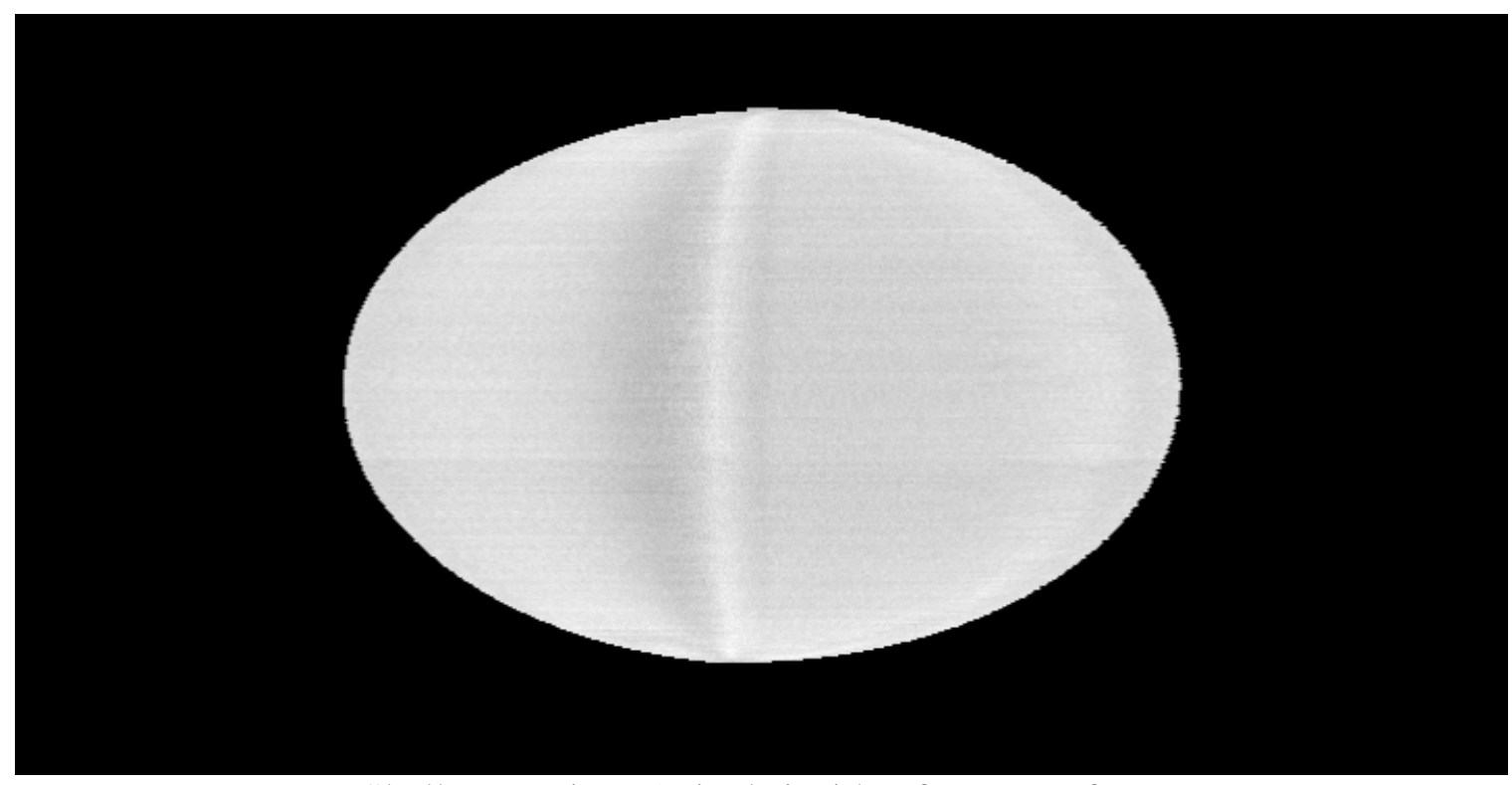

Shell extraction, 5 pixels inside of outer surface. 
SN 6-92-017

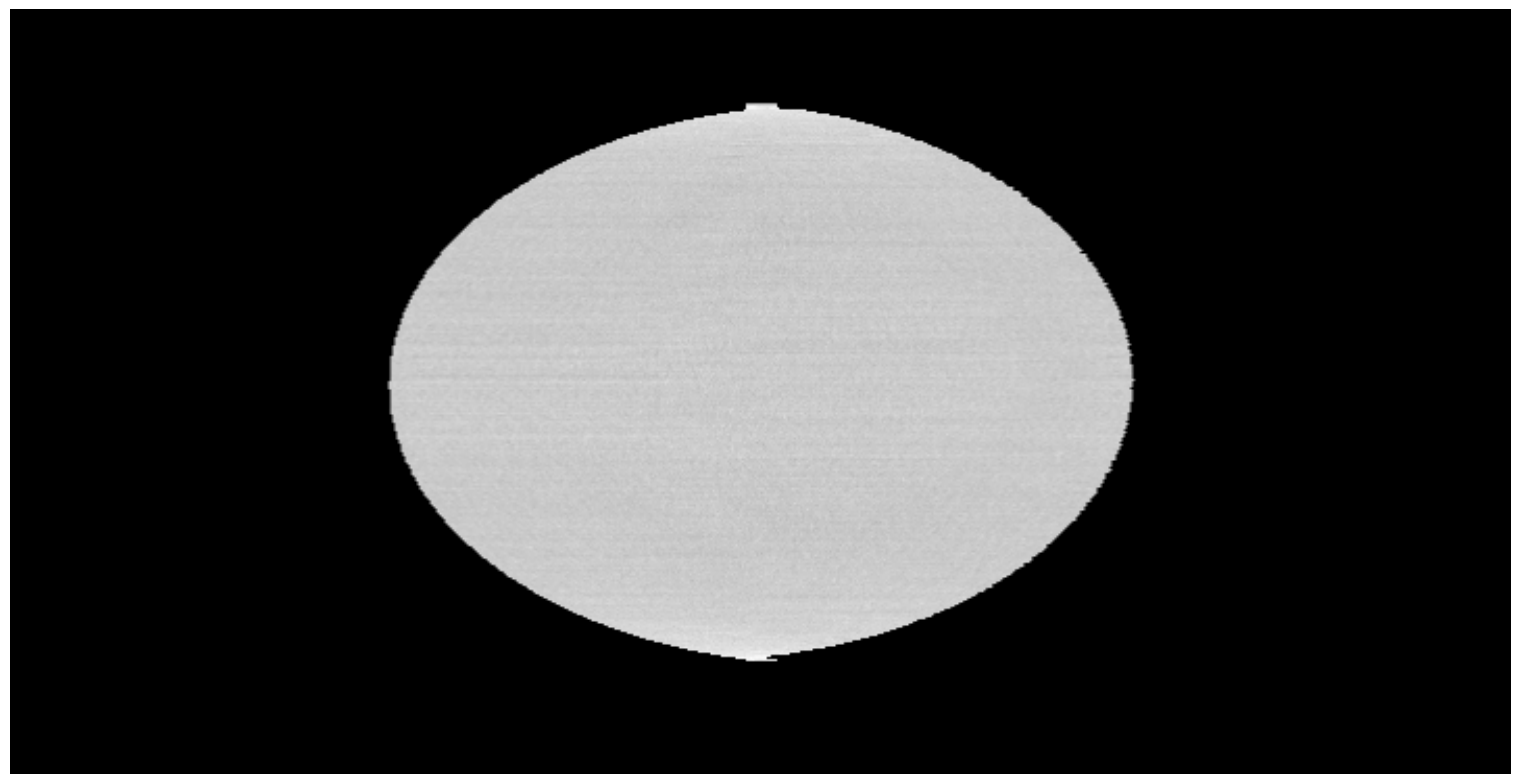

Shell extraction, taken 20 pixels inside of outer surface.

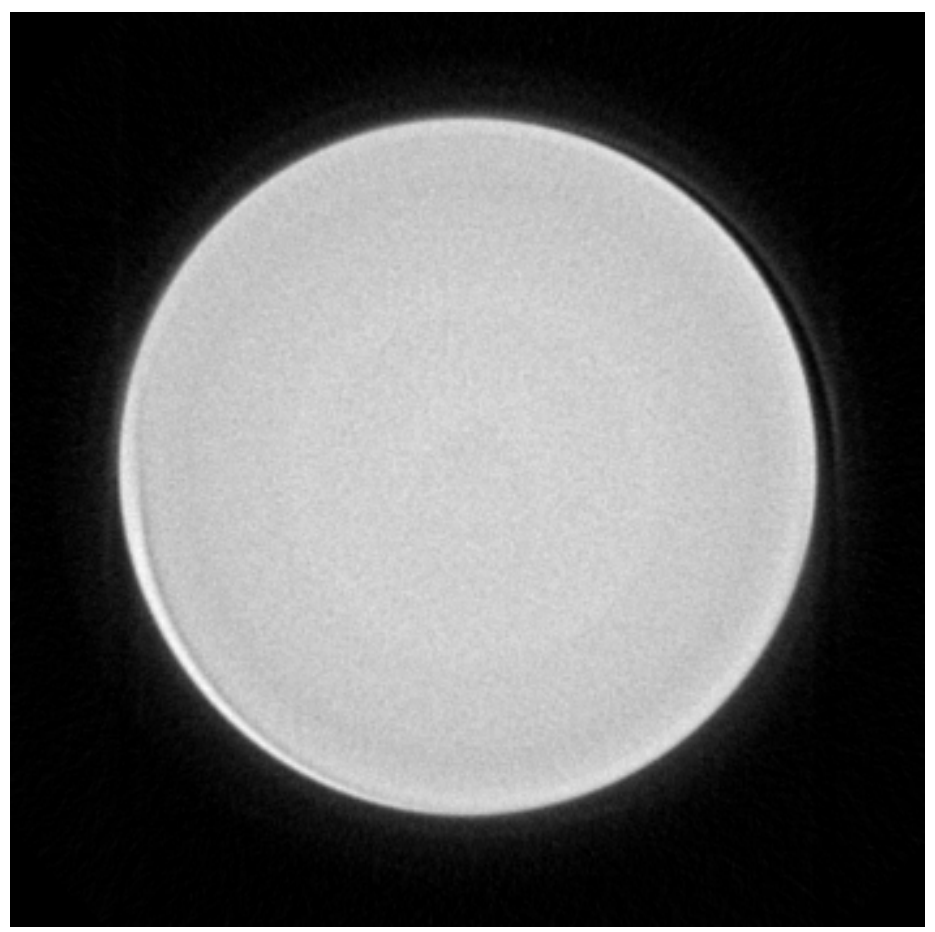

Z slice average of slices 223-227

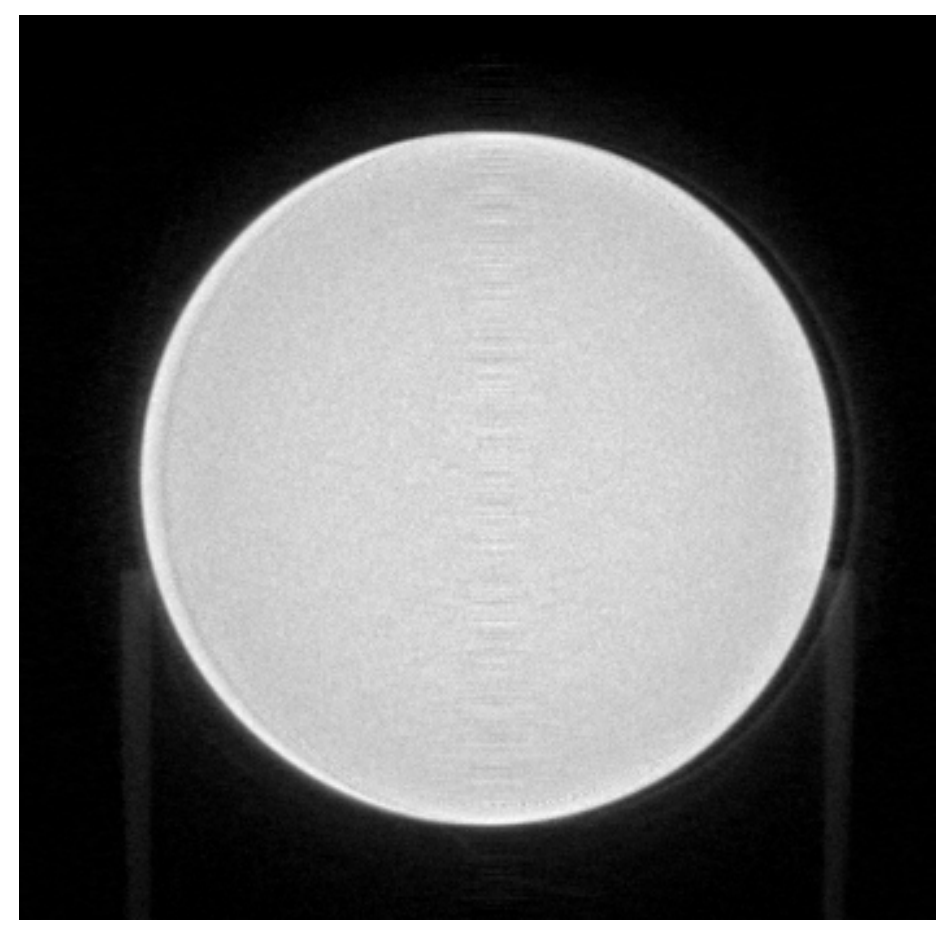

Y slice average of slices 223-227. 